Illinois State University

ISU ReD: Research and eData

Theses and Dissertations

6-23-2021

\title{
Investigating Intersubjectivity Between Preschool Teachers and Children During Instruction: A Case Study
}

Andrianna Eleni Smyrniotis

Illinois State University, adragonteacher@gmail.com

Follow this and additional works at: https://ir.library.illinoisstate.edu/etd

\section{Recommended Citation}

Smyrniotis, Andrianna Eleni, "Investigating Intersubjectivity Between Preschool Teachers and Children During Instruction: A Case Study" (2021). Theses and Dissertations. 1472.

https://ir.library.illinoisstate.edu/etd/1472

This Dissertation is brought to you for free and open access by ISU ReD: Research and eData. It has been accepted for inclusion in Theses and Dissertations by an authorized administrator of ISU ReD: Research and eData. For more information, please contact ISUReD@ilstu.edu. 


\title{
INVESTIGATING INTERSUBJECTIVITY BETWEEN PRESCHOOL TEACHERS AND CHILDREN DURING INSTRUCTION: A CASE STUDY
}

\begin{abstract}
ANDRIANNA E. SMYRNIOTIS
156 Pages

Early childhood teachers attend professional development sessions, take college-level courses, or read articles, and will, at some point, be exposed to some concepts found in Vygotsky’s (1986) cultural-historical theory of cognitive development as they are deeply embedded in education standards, professional standards, texts, and journals throughout the country. And yet, some concepts, including scaffolding (Wood et al., 1976), are the result of misinterpretations and the assignment of a label over the years (Gonzales Rey (2008). A largely overlooked concept accurately relating to Vygotsky's work is intersubjectivity (Trevarthen, 2008), defined as mutual understanding, among teachers and children, for concept development. Intersubjectivity is reached outside the zone of proximal development (ZPD) by assessing prior knowledge and within the ZPD while engaging children through guided participation (Vygotsky, 1986). Learning why this is important and how to achieve intersubjectivity through guided participation is of critical importance to pre- and in-service professionals. Guided participation is child-centered pedagogy involving multi-modal communication to reach intersubjectivity. The process can be explained as an interactive 'dance' between a more knowledgeable other (MKO) and the learner that may include verbal language, gestures, facial expression, and props so they can both to reach mutual understanding (Brinck, 2008; Vygotsky, 1986).
\end{abstract}

The purpose of the study was to investigate preschool teachers' engagement with children, communicating toward intersubjectivity (mutual understanding) during the learning 
process, in preschool classrooms. In many preschools, there tends to be an 'either-or' emphasis on teacher-directed learning (more academic) or child-directed (play-based) learning. Because the concept of mutual understanding relies on give-and-take (aka serve and return) verbal and non-verbal communication, this study examined practices of three participants in play-based programs serving children from three to five years of age.

In this qualitative, phenomenological case study, the methods used to gather data were classroom observations, semi-structured interviews, video of the classroom environments, and coded analysis of data. Findings revealed that two of the three participants used more direct instruction with one leaning more toward intersubjective instruction through guided participation and mediated activity.

KEYWORDS: Preschool, Vygotsky, Intersubjectivity, Guided Participation, Zone of Proximal Development 
INVESTIGATING INTERSUBJECTIVITY BETWEEN PRESCHOOL TEACHERS AND

CHILDREN DURING INSTRUCTION: A CASE STUDY

ANDRIANNA E. SMYRNIOTIS

A Dissertation Submitted in Partial

Fulfillment of the Requirements for the Degree of

DOCTOR OF EDUCATION

School of Teaching and Learning

ILLINOIS STATE UNIVERSITY

2021 
Copyright 2021 Andrianna E. Smyrniotis 
INVESTIGATING INTERSUBJECTIVITY BETWEEN PRESCHOOL TEACHERS AND

CHILDREN DURING INSTRUCTION: A CASE STUDY

ANDRIANNA E. SMYRNIOTIS

COMMITTEE MEMBERS:

Amanda Quesenberry, Chair

Alan Bates

Terry Husband 


\section{ACKNOWLEDGMENTS}

...if I like to convey the feeling of cold, I may do this with the help of expressive gestures, but real understanding and communication will be achieved only through generalization and conceptual designation of my experience. Such generalization would refer my experience to the class of phenomena known to my interlocutor. That is why certain thoughts cannot be communicated to children even if they are familiar with the necessary words. The adequately generalized concept that alone ensures full understanding may still be lacking.

$$
\text { Vygotsky, 1986, p.8 }
$$

The sense of a word...is the sum of all the psychological events aroused in our consciousness by the word. It is a dynamic, fluid, complex whole, which has several zones of unequal stability. Meaning is only one of the zones of sense, the most stable and precise zone. A word acquires its sense from the context in which it appears; in different contexts it changes its sense.

Vygotsky, 1986, pp. 244-45

Children learn in the context of meaningful relationships.

Rima Shore, 1997, p. 33

To Dad, Jim, Greg, Christopher, Paul, and Andrew; you justify what I do and the passion with which I express it. I kept my promise.

A.E.S. 


\section{CONTENTS}

Page

ACKNOWLEDGMENTS

CONTENTS

FIGURES

CHAPTER I: INTRODUCTION 1

Purpose of the Study 1

Significance of the Study $\quad 5$

$\begin{array}{ll}\text { Research Design } & 10\end{array}$

Definition of Terms 11

$\begin{array}{ll}\text { Conceptual Framework } & 15\end{array}$

Theoretical Framework and Research Paradigm 21

Context of the Study 22

$\begin{array}{ll}\text { Summary } & 25\end{array}$

CHAPTER II: LITERATURE REVIEW 26

$\begin{array}{ll}\text { Introduction } & 26\end{array}$

$\begin{array}{ll}\text { Problem Statement } & 28\end{array}$

Vygotsky’s Cultural-Historical Theory of Cognitive Development 28

The Zone of Proximal Development 30

Concept Development 32

Guided Participation / Mediated Activity 35

$\begin{array}{ll}\text { Intersubjectivity } & 38\end{array}$

$\begin{array}{ll}\text { Summary } & 46\end{array}$ 
$\begin{array}{ll}\text { CHAPTER III: METHODOLOGY } & 48\end{array}$

$\begin{array}{ll}\text { Introduction } & 48\end{array}$

$\begin{array}{ll}\text { Problem Statement } & 48\end{array}$

Purpose of the Study and Research Questions 52

Research Design: Phenomenological Instrumental Case Study $\quad 54$

Participants and Setting of the Study

$\begin{array}{ll}\text { Data Collection } & 57\end{array}$

$\begin{array}{ll}\text { Data Analysis } & 60\end{array}$

Ethical Issues: The Role of the Researcher 62

$\begin{array}{ll}\text { Summary } & 64\end{array}$

$\begin{array}{lc}\text { CHAPTER IV: FINDINGS } & 67\end{array}$

$\begin{array}{ll}\text { Teacher Demographics } & 68\end{array}$

Teacher Perceptions of Guided Participation/Mediated Activity $\quad 69$

$\begin{array}{ll}\text { Denise } & 69\end{array}$

$\begin{array}{ll}\text { KA } & 71\end{array}$

$\begin{array}{ll}\text { Ann } & 76\end{array}$

$\begin{array}{ll}\text { Summary } & 78\end{array}$

Intersubjective Communication to Assess Readiness and Engage Learning 80

in the ZPD

$\begin{array}{ll}\text { Denise } & 83\end{array}$

$\begin{array}{ll}\text { KA } & 91\end{array}$

$\begin{array}{ll}\text { Ann } & 97\end{array}$

$\begin{array}{ll}\text { Summary } & 108\end{array}$ 
$\begin{array}{ll}\text { Conclusions } & 110\end{array}$

CHAPTER V: DISCUSSION 116

$\begin{array}{ll}\text { Guided Participation/Mediated Activities } & 117\end{array}$

$\begin{array}{ll}\text { Intersubjectivity and ZPD } & 119\end{array}$

$\begin{array}{ll}\text { Limitations of the Study } & 127\end{array}$

$\begin{array}{ll}\text { Implications for Future Research } & 129\end{array}$

Future Research to Expand Standards and tools relating to high quality 130

ECE professionals

Future Research Methods $\quad 132$

Implications for Practice $\quad 133$

Implications for Professional Assessments and Preparation Standards 134

Implications for Professional Development and Teacher Education Programs 135

$\begin{array}{ll}\text { Concluding Reflections } & 138\end{array}$

$\begin{array}{ll}\text { REFERENCES } & 141\end{array}$

APPENDIX A: INTERVIEW ONE SCRIPT AND QUESTIONS 155

APPENDIX B: INTERVIEW TWO AND THREE SCRIPT AND QUESTIONS 156 


\section{FIGURES}

Figure $\quad$ Page

1. Conceptual Funnel of Guided Participation 20

2. Process of Intersubjective Instruction 20

3. Baby Animal Pattern $\quad 84$

4. Establish Prior Knowledge, Then Enter the ZPD 123 


\section{CHAPTER I: INTRODUCTION}

\section{Purpose of the Study}

In Illinois, general practices in early childhood education tend to be generalized, in that there is no one approach, or curriculum being exercised. It is rare to see a 'purely' High Scope, Project Approach, or Reggio classroom. The most influential guidance for non-specifically trained educators has been from the National Association for the Education of Young Children (NAEYC), the largest early childhood education organization in the United States. NAEYC's mission ("Our Mission and Strategic Position," n.d.) is to "promote high quality early learning for all children, birth through age eight, by connecting practice, policy, and research." They offer accreditation to licensed programs that successfully complete a rigorous assessment and evaluation that occurs over time and must be renewed at regular intervals ("NAEYC Accreditation: Early Learning Programs," n.d.). Given the recognition of NAEYC as a resource of high quality, there are 411 NAEYC accredited programs identified in March 2018, in Illinois ("Search NAEYC Accredited Programs," n.d.). The number of total child care centers in Illinois is 4,001 ("childcarecenter.us/state," n.d.). If the number of centers stayed relatively constant, this would mean $10.2 \%$ of all centers in Illinois are NAEYC accredited. By comparison, and from the same sources, Wisconsin has 4.5\% NAEYC accredited centers (2570 centers and 117 accredited), and Indiana has 14.4\% NAEYC accredited centers (1640 centers and 237 accredited).

Over fifty years of extensive study on early childhood care (Helburn, 1995; Jalongo et al., 2004; Weikart, 1967/2016) and brain research (The National Research Council and Institute of Medicine report, 2000; Shonkoff \& Phillips, 2000; Shore, 1997) brought the need for high quality early childhood care and education to the attention of local, state, and the federal 
government. A preponderance of research regarding the attributes of quality on child development led the federal government to pass the No Child Left Behind (NCLB) Act of 2001 (“H.R.1-No Child Left Behind Act of 2001," n.d.). NCLB influenced policy initiatives tied to early childhood education that include compiling appropriate learning standards and assessments (Stipek, 2006). As a result, pre-K programs felt push-down pressure to teach academics - a practice that waxes and wanes over the years.

The Race to the Top Early Learning Challenge (Race to the Top-Early Learning Challenge, 2016) was a competitive grant made available to states with focus on improving early learning and development programs, and required all states writing proposals to develop a Quality Rating and Improvement System (QRIS). Illinois named its QRIS ExceleRate (What is ExceleRate, n.d.), and uses a government-sponsored website to report all licensed child care centers and homes' level of quality, as determined by standards and a framework of quality and improvement factors that includes teacher level of education (ExceleRate Illinois Standard and Evidence Requirements, n.d.). Assessed quality will be acknowledged with designations of circles of excellence, from the lowest (state licensing), to bronze, silver, and gold circles (ExceleRate Illinois Program Overview, n.d.). Incentives to improve quality includes higher monetary reimbursement (ExceleRate Illinois Incentives for Center Based Programs, n.d.) for children enrolled under the Child Care Assistance Program (CCAP). Program improvement includes raising the training/education level of early childhood teachers. Over time, teachers who earned some college credits will be required to return and continue work on certificates, associate degrees, or bachelor's degrees in early childhood education (ExceleRate Illinois Quality Standards Overview, 2017). Personal conversations with center directors and business owners indicate a real concern for continued operation of child care services with teachers 
holding bachelor's degrees, as the additional income for CCAP children will not cover demand for higher wages by early childhood professionals with greater credentials. Families who already pay a substantial percentage of their income for child care are not expected to be able to pay more and more tuition. This poses a real and imminent concern for quality child care delivery in Illinois.

Given the increases in requirements for early childhood teachers, this leads us to the question, are expectations of high-quality preschool being met by high quality teachers? Currently, Illinois requires all teachers of the state's public-funded preschool program, Preschool for All (PFA) to hold a bachelor's degree in early childhood education (ECE). According to the Illinois State Board of Education Illinois Teacher Salary Study 2014-2015 (Illinois State Board of Education Data Analysis Division, 2015), the lowest starting salary paid to an inexperienced, licensed teacher with a bachelor's degree is $\$ 20,343$, with the median starting salary being $\$ 36,927$. In contrast, and according to the Illinois Department of Human Services (Illinois Salary and Staffing Survey of Licensed Child Care Facilities Fiscal Year, 2015), the median hourly wage for a full-time early childhood teacher was $\$ 12.00$ per hour, which is approximately $\$ 24,960$ per year. Reported results are based on respondents from all licensed facilities, which may include Head Start.

Figures are difficult to effectively compare, as different reporting entities may categorize the workforce in different ways. In its latest fact sheet of salaries, the U.S. Department of Education listed the annual (2015) median salaries in Illinois for child care workers to be \$21,830 (Fact Sheet: Troubling Pay Gap for Early Childhood Teachers, 2016). There is no distinction between lead and assistant teachers. In contrast, the median income of preschool teachers in Illinois is listed as $\$ 28,670$, which includes PFA teachers yet does not include Head 
Start teachers, whose median salary in 2015 is reported to have been $\$ 32,691$. Also included in the report is the median salary of kindergarten teachers in Illinois, $\$ 48,710$, who have the same requirements as PFA teachers.

Whether a lead teacher is paid minimum wage or higher salaries, they are all expected to have some degree of higher education training. Current trends indicate that higher quality ECE teachers hold BA degrees in ECE or Child Development (Institute of Medicine and National Research Council, 2015). Does a four-year degree reflect practical knowledge of intersubjective communication in practice if not through knowledgeable use of the term? This study may unveil information on this topic, as relational communication is considered a major strength for effective teaching (Bruner, 1990; Pianta et al., 2008; Vygotsky, 1986), and intersubjectivity is relational communication in a cultural context. Other factors to determine quality include a program that is NAEYC accredited, one that holds a gold circle of excellence awarded by ExceleRate Illinois, or one that focuses on a specific, progressive model of inspiration such as the Reggio Emilia approach to ECE. The concept of intersubjectivity is further discussed in the next section.

The purpose of the study was to investigate preschool teachers' interactive instructional practices with three to five-year olds, and the methods of intersubjective communication they use. Intersubjective communication can be explained as an interactive 'dance' between a more knowledgeable other (MKO) and the learner that may include verbal language, gestures, facial expression, and props so they can both to reach mutual understanding (Brinck, 2008; Vygotsky, 1986). Understanding is not the same for all children and teachers, and culture plays a major role as to whether it is reached. For example, children with limited vocabulary or experiences may be challenged to understand certain words used by a teacher. Will she use a child's own 
experiences, lexicon, props, gestures, or examples to strengthen the chance she will be understood?

\section{Significance of the Study}

Early childhood practitioners attend mandatory, professional development trainings and take early childhood courses that inspire them to exercise different instructional and behavioral strategies, methods, and activities in their classroom. Many will take their newfound competence back to their programs with intentions of implementing effective strategies with their students the next school day. However, what actually tends to happen is that a teacher implements fragments of an approach or curriculum without benefit or understanding of the entire vision, which compromises the integrity of the approach and ultimately, the quality of her professionalism (Buysse et al., 2009). One concept that is often taught without full understanding, and therefore misinterpreted and implemented without fidelity, is Vygotsky's explanation of constructing knowledge that has become known as 'scaffolding.'

Whether early childhood teachers take a training workshop, a college-level course, or read articles, they will, at some point, be exposed to the metaphor of scaffolding and the zone of proximal development (ZPD). Vygotsky's (1986) cultural-historical theory of cognitive development is deeply embedded in education standards, professional standards, texts, and journals throughout the country. And yet, there is one critical component of scaffolding a child's cognitive development in the ZPD that eludes almost every preschool teacher that I have observed in more than twenty years. This critical component is intersubjectivity. According to Vygotsky's theory, teachers must engage a learner in intersubjective discourse to first, determine a child's level of independent abilities, and second, to mediate conceptual contexts with a child in his zone of proximal development. Engagement is key, as it means there is a true back and 
forth of communication that includes being present and listening and is multidimensional rather than strictly verbal.

Vygotsky $(1978,1986)$ explained the concept of engagement between more knowledgeable others (MKOs) and infants/children as a communicative process that leads to mutual understanding, incorporating this dynamic as critical for two reasons; to determine what a child can do without assistance, and to be constant throughout assistance in the ZPD (Vygotsky, 1986). Vygotsky never used the terms 'scaffolding,' or 'intersubjectivity.' The moniker 'intersubjectivity' was first used by Trevarthen (2008) in 1974 and is widely found in the discipline of linguistics and communication, with very little representation in education. Trevarthen admits, however, to borrowing the term from Joanna Ryan who, it seems, coined the term to describe "the development of 'communicative competence' before language" (Trevarthen, 2008, p. ix). Intersubjectivity applies to all ages, as communication is a complex undertaking. Indeed, understanding itself is a process, not as elementary as defining terms and labeling things. For example, Vygotsky (1986) wrote,

...if I like to convey the feeling of cold, I may do this with the help of expressive gestures, but real understanding and communication will be achieved only through generalization and conceptual designation of my experience. Such generalization would refer my experience to the class of phenomena known to my interlocutor (p. 8).

Research and knowledge about intersubjectivity is largely found outside the United States and within the discipline of linguistics, communication, educational psychology, and psychology. Even then it is rare to find references to intersubjectivity as it relates to preschool level education. 
In relation to preschool children, ages three to five, intersubjectivity addresses the multimodal tools of communication that includes language between 'teacher' and 'learner' to come to mutual, collaborative understanding. Preschool children are new to the abstractions of words (concepts). To internalize and eventually generalize these concepts, young children rely as much on expression, tone, gestures, artifacts/evidence to amass a cache of references to that concept. In other words, according to Vygotsky (1986), young children have preconcepts until they have stored enough scope and reference to that concept that they truly understand it in general terms. Through understanding, teachers as well as children make meaning, scaffold their knowledge, and expand their conceptual knowledge.

The words 'clarify,' 'explain,' and 'use examples' help teachers understand their role in collaborating toward intersubjectivity to some extent. If one believes Vygotsky's (1986) theory that learning is cultural and social, there must be a point in which teacher communicates with a child to ascertain prior knowledge demonstrated in more than words, then adjust their own knowledge to that of the child to move forward, using communication in its broad sense. Without engagement toward mutual understanding, there will be no meaningful scaffolding in the zone of proximal development (ZPD). For children to develop higher mental processes, mediation concepts such as intersubjectivity and scaffolding are crucial (Vygotsky, 1997a).

Reading more and widening my scope of understanding this abstract idea, I came to learn that there are references to the concept of intersubjectivity in education that are embedded within terms that may or may not also mention the words 'scaffold,' and the term 'ZPD.' Vygotsky's work did not benefit from a long research career with more publications to further clarify his work (Gredler \& Shields, 2008; van der Veer \& Valsiner, 1991), and so subsequent research may further clarify, dilute, or change his original concept. In the United States, Wood et al (1976) 
added the constructive metaphor of scaffolding into Vygotsky's writings on the ZPD, leaving a debate as to whether the metaphor is appropriate and/or accurate (LaJoie, 2005; Palinscsar, 1986; Stone, 1998), or whether educators' perception of scaffolding is accurate (Searle, 1984; Verenikina, 2004). Related terminology includes meaning making/shared meaning (Bruner, 1990; Dahlberg et al., 1999; Flint, 2010; Fredriksen, 2010), guided participation (Rogoff, 2008; Rogoff et al., 1993, participatory appropriation (Rogoff, 2008), negotiated meaning (Gjems, 2010; Luke \& Kale, 1997; Müller, 2003), co-construction of knowledge (Jordan \& Smorti, 2010; Moss, 2006; Valsiner, 1996); appropriation of meaning (Wertsch \& Stone, 1985); and dialogic pedagogy (Daniels, 2014). Such a variety of terms is likely to cause confusion about intersubjectivity or appear as completely separate concepts to early childhood professionals. Ineffective interactions or confusion may occur when discussions of terms other than intersubjectivity mention dialogue or conversation yet fail to impress the importance of nonverbal communication with young children. While Vygotsky (1986) wrote of the importance of gestures, he stressed the understanding of the concept. Very young children will understand concepts in a deeper way when words are accompanied with gestures, props, demonstrations, expressions, and body language, as we learn from research on English language learners (WIDA, 2021).

Demographics in the United State indicate that, by the year 2030, and probably sooner, Latino, Asian, and African American students, will make up the majority of public school children (Center for Public Education, 2012), and the majority of teachers continue to be middle class women of European ancestry (National Center for Education Statistics, 2009). Culture, poverty, language, and ability are all factors creating divide between teacher and student, as well as families. This makes the need for effective communication - for intersubjectivity - more 
important; so children are understood and instructed to reach their full potential rather than mistakenly considered slow, or worse, unable to learn. Teachers who are unaware of intersubjectivity, through practice if not by term, will be much less effective in the classroom.

Early childhood education and child development textbook authors (Berger, 2018; Berk \& Meyers, 2021; Kostelnik et al., 2015) recognize the value and importance of Vygotsky's cultural-historical theory of cognitive development and, wherever it is discussed in text, the concepts of scaffolding and ZPD are prominent. All mention the importance of 'interaction' while scaffolding knowledge. However, that is where the information ends. Creative teaching (Mayesky, 2011) does not express the importance of dialogue or conversation other than touching on open-ended questions and getting to know the children's interests. Intersubjectivity, mutual understanding for young children, requires a teacher's use of multi-modal communication and the development of deep relationships with children. Emphasis must be made to stress the back and forth of interactions, from teacher to child to teacher, and so on. Teachers who ask, "Do you understand?" and receive an answer of "Yes," are not engaging toward intersubjectivity, as it is one-sided, and teacher-directed.

Intersubjective communication is more complex than two people involved in (openended) dialogue toward mutual understanding. Teachers have a responsibility to consider their own culture and the cultures of the child(ren) they teach, as it is the basis of a person's experiences, thinking, and behaviors (Rogoff, 2008). Knowledge of this dynamic obligates teachers to have an extensive understanding of themselves, and to be inquisitive and adaptive thinkers. Coming to mutual understanding with children will greatly change the instructional approach of many preschool teachers. 
Constructed meaning, negotiated meaning, co-construction of knowledge, participatory appropriation, negotiated, renegotiated, and mediated meaning, and shared meaning are all terms used to loosely define intersubjectivity. There is need to apply a comprehensive definition to the term intersubjectivity (without changing it) so that educators can learn, practice, and include its practice when scaffolding learning.

There is continued interest in effectively teaching children from birth to 6 years. One of the hot topics of public education is improving learning and developmental outcomes, and there is a fear that academic drilling to skill is going to be the norm in preschools. Cindy Zumwalt, Early Childhood (EC) Division administrator at the Illinois State Board of Education (ISBE) gave a presentation to the Higher Education Early Childhood Faculty Forum in Bloomington, Illinois, on April 9, 2015 (Zumwalt, 2015). Reporting the results of the latest scores on the ECERS, Early Childhood Environment Rating Scale (Harms, Clifford, \& Cryer, 2015), for Preschool for All (PFA) teachers, all of whom must hold a bachelor's degree with certificate/license in early childhood education, there was an outcome that was particularly concerning. The average score under the category, 'using language to develop reasoning skills' for PFA teachers was 3.6 out of 7 possible points. This score is relevant to this study because the ability to reason develops from intersubjectivity, due in part to concept development and the mutual plane of understanding between teacher and child.

\section{Research Design}

The nature of this qualitative study was phenomenological and, as such, required me to objectively describe and interpret the perceptions, behaviors and thoughts of others (Glesne, 2011). In addition to interpreting information, I constructed the study, developing subjective meanings from my investigations. I also relied on the teachers' and children's behaviors to 
construct deeper understanding of intersubjective communication through observed interactions, the teachers' explanations of their instructional practices, and analysis of the classroom environments. As a result of the teacher-researcher interactive communications, they too, reached intersubjective understanding. "Often these subjective meanings are negotiated socially and historically.... they are not simply imprinted on individuals but are formed through interactions with others (hence social constructivism) and through historical and cultural norms that operate in individuals' lives" (Creswell, 2007, p.21). My understanding is shared in this paper with as much description as possible. Yet, no matter how complete the research may seem, no two people will understand the information in the same way.

\section{Definition of Terms}

For the purposes of this study, it is important to acquire a consistency and clarity of terms as they apply to development at the preschool level, three to five years, including:

Close-ended question - a question that requires an absolute response, such as 'yes,' 'no,' 'this one,' or 'a triangle.'

Communication - Preschool children are learning verbal and written language and so, along with written and spoken language comes non-verbal behaviors that both children and teachers employ in order to truly understand each other. Gestures, facial expressions, voice intonation and inflection, body language, signs, and listening are integrated to reach understanding about feelings, thoughts, and meanings.

Concept - according to Merriam-Webster Dictionary-Thesaurus, 2017, synonym discussion:

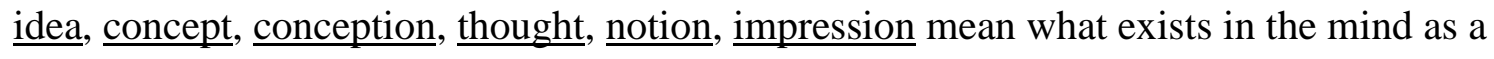
representation (as of something comprehended) or as a formulation (as of a plan). Idea may apply to a mental image or formulation of something seen or known or imagined, to a pure 
abstraction, or to something assumed or vaguely sensed <innovative ideas> <my idea of paradise >. Concept may apply to the idea formed by consideration of instances of a species or genus or, more broadly, to any idea of what a thing ought to be < a society with no concept of private property $>$. Conception is often interchangeable with concept ; it may stress the process of imagining or formulating rather than the result < our changing conception of what constitutes art >. Thought is likely to suggest the result of reflecting, reasoning, or meditating rather than of imagining <commit your thoughts to paper〉. Notion suggests an idea not much resolved by analysis or reflection and may suggest the capricious or accidental <you have the oddest notions $>$. Impression applies to an idea or notion resulting immediately from some stimulation of the senses <the first impression is of soaring height > (Merriam-Webster DictionaryThesaurus, 2017).

Construction \& Reconstruction - using a combination of speech, materials/action, and context to construct concepts, and then, to co-construct and reconstruct concepts in the development of higher psychological functions; It is the process of concept development (Vygotsky, 1986, Vygotsky \& Luria, 1994).

Dialogue - The interest in this study is for continuous, communicative discussion - a conversation between two or more interlocutors. Merriam-Webster Dictionary-Thesaurus (2017) definition $2 \mathrm{~b}$ for dialogue is "an exchange ideas and opinions." Asking a close-ended question and receiving a response is not considered a dialogue.

Frustration - Merriam-Webster Dictionary (2018) provides this definition for English language learners, applicable in this context:

-a feeling of anger or annoyance caused by being unable to do something: the state of being frustrated 
-something that causes feelings of anger and annoyance

-the fact of being prevented from succeeding or doing something

Guided participation - A process of systemic cooperation and systemic instruction between child and teacher, "affected by varying external and internal conditions," leading to the development of concept formation. "Instruction is one of the principal sources of the schoolchild's concepts and is also a powerful force in directing their evolution; it determines the fate of his total mental development (Vygotsky, 1986)." Used in partnership with 'mediated activity,' Vygotsky alluded to these terms as effective instructional practice in the zone of proximal development. Wood et al (1976) coined the term 'scaffolding.' Interpretation and reinterpretation of the term 'scaffolding' over the years strays far afield from Bruner's definition. As a result, this research will stay true to Vygotsky's terms and explanations.

Instruction - The act of guided participation and mediated activity to develop concepts Interaction - communication that includes dialogue between two or more interlocutors. Interlocutor - a person who participates in discussion, dialogue, conversation. Intersubjectivity - In this context, intersubjectivity will include the communicative interactions as well as the point at which mutual understanding is reached. The process involves sharing current understanding, sharing new concepts through shared understanding, and mutual understanding of the new concepts using contexts familiar to the child. Vygotsky used the phrase mediated understanding. Guided participation is the process to reach intersubjectivity.

Language - according to Merriam-Webster, "the words, their pronunciation, and the methods of combining them used and understood by a community."

Listening - being present, using eye contact, non-verbal communication to acknowledge what a speaker is saying. Listening does include verbal acknowledgements of what is said, and seeks 
clarification, perhaps through examples, demonstration or other signs. Listening is an element of dialogue and communication.

Mediate - according to Merriam-Webster:

a : to bring accord out of by action as an intermediary

b : to effect by action as an intermediary

$\mathrm{c}:$ to act as intermediary agent in bringing, effecting, or communicating : convey

$\mathrm{d}:$ to transmit as intermediate mechanism or agency

e : to interpose between parties in order to reconcile them

$\mathrm{f}:$ to reconcile differences

Mediated activity - according to Vygotsky (1978) mediated activity is 'indirect' in the context of using signs (verbal \& non-verbal communication) and tools, i.e. objects, to explore and experience concepts. "Both tool and sign are used mutually linked and yet separate in the child's cultural development (p. 54)". However, "cognitive activity is not limited to the use of tools or signs" (Vygotsky, 1978, p. 55). Therefore, mediated activity is equally paired with guided participation, and in lieu of the term 'scaffolding.'

More knowledgeable other (MKO) - While in the zone of proximal development (ZPD), "learning is social in origin and it happens in the presence of others that are more knowledgeable" (Abtahi et al., 2017).

Open-ended question - questions that require more thought; teachers utilizing open-ended questions get a sense of what a child is thinking, feeling, intending, for example.

Scaffolding - the term will be used sparingly in this study; refer to coconstruction/reconstruction of knowledge using guided participation or mediated activity. 
Semiotics - according to Merriam-Webster Dictionary-Thesaurus (2017) is "a general philosophical theory of signs and symbols that deals especially with their function in both artificially constructed and natural languages and comprises syntactics, semantics, and pragmatics."

Sign - 1: Any object, action, event, pattern etc. that conveys a meaning (Rodriguez \& Moro, 2008). 2: a gesture or action used to convey information or instructions.

Zone of proximal development - the place in a learner's cognitive development between what she can do independently, and what she cannot do by independently.

\section{Conceptual Framework}

This study explored preschool teacher-child intersubjective engagement in the context of intentional, multi-modal communication, in early childhood environments for the purpose of reaching intersubjectivity, or common understanding, during instruction outside and within the ZPD. As Vygotsky and his successors pointed out, mutual understanding is a crucial element of concept development, necessary for problem solving and knowledge building (Vygotsky, 1978; Zlatev et al., 2008). A teacher must engage (expressing and listening) as much as the child to learn what the child is thinking about the concept(s) being presented. Through this intersubjective communication, teachers learn and verify a child's independent capabilities. As a child is taken into the Zones of Proximal Development, teachers must be careful not to push a child too hard regarding concept development. Excessive frustration can lead a child to resist learning or dislike school altogether (Vygotsky, 1986). Key concepts this study focused on were concept development, guided participation, intersubjective communication, the zone of proximal development (ZPD), and frustration. This last concept is important for teachers to note, as 
Vygotsky (1986) clearly mentions the danger that continued frustration may influence a child to lose his love of learning.

A general illustration of guided participation between a preschool teacher and student is offered in Figure 1. Using the funnel illustration, one can see that guided participation uses intersubjective communication methods to assess prior knowledge, and to engage a child during instruction in the ZPD. It is important for teachers to assess the level of prior knowledge, which includes a child's ability to make generalizations about a concept, prior to taking a child into the ZPD. For example, a three-year-old may look at a shape book and point to a rectangle and correctly identify the shape. If teacher has several paper shapes on the table as well, the child may, again, correctly identify the rectangle. This does not mean the child is able to generalize 'rectangle' in other contexts. Perhaps he is not able to see a book or a window as a rectangular shape. Perhaps her only experience with 'rectangles' is in the context of cut-out paper shapes. If that is the case, the teacher will take the child into the ZPD and using guided participation, show her the attributes of rectangles, using verbal and non-verbal communication, expressing her understanding of the concept and drawing from her own experiences. The teacher will use references to the child's culture with items that may include particular toys, windows, boxes, etc. Teacher is in a position to build upon the child's knowledge as well as her own, all the while continuing with intersubjective discourse (verbal and non-verbal). After some demonstration, the child may be asked to find another rectangle in the classroom. The child will be given time to ponder and, if she appears frustrated, teacher will kindly assist, perhaps to remind her that rectangles have four straight sides. If successful, the teacher may request identification of another rectangle, reinforcing the child's successes through the process with specific acknowledgement. Both teacher and child are expressing feedback (e.g., verbal, demonstration, 
modeling, gestures, etc.) as the construction of new knowledge through experience percolates. When the child has a few successful identifications, teacher will step back for the child to continue on her own or move to another activity. If the child demonstrates frustration, she must be allowed to go to another activity. Whether it is a child who went into the ZPD, or one that has yet to enter at that level, teacher should make notes to be prepared for the next time they engage in rectangles. The process is illustrated in Figure 2.

Without seeking mutual understanding of concepts, teachers are apt to feel they are teaching when they are not. For example, a middle-class preschool teacher, having grown up in a neighborhood of single-family homes, near a park and safe from neighborhood violence, may prepare a pretend picnic for the children that they may have in their yard, or the park. She may set the scene in the dramatic play area of the classroom, and join the dramatic play, talking about how fun it is to go to the park or one's yard to have a picnic on a warm day. The children are enjoying eating pretend food that is set on a tablecloth on the floor. They may be practicing their manners or talk to their teacher about how they like hot dogs and corn. In this example, teacher said, "I like picnics in the summer." She made acknowledgments of children's comments yet did not ask open-ended questions. After the play wound down, the teacher, happy that the children 'enjoyed' the activity, writes, "The children enjoyed a pretend picnic today" on her daily notes to parents.

As some children are being picked up, a mom asks, "what did you do today?" The child answers, "we ate on the floor.” Another mom comments, “Oh, you had a picnic?" The child responds, "what?" Mom repeats, “did you have a picnic?” The child replies, "I don't have a picnic." This example is based on a true teacher observation made during my years as a center director. When we met to discuss her professional development, this example was brought up, 
and the teacher indicated she was very happy to have engaged with the children. I asked if she talked with the children about picnics, and she indicated that she had. It turned out that she talked to the children about picnics and did not take time to learn about the children's own concept of 'picnic' based on their own histories. Children who lived in unsafe neighborhoods did not have experiences to eat in the yard (they may not have a yard), or at the park, as it is too dangerous due to drug deals, discarded paraphernalia, and potential violence. Other children have met friends and families at a park, although they did not use the word, 'picnic.' Given these realities, even if the teacher successfully identified the pretend activity to the children, they likely would think a picnic means eating pretend food on the floor at school until other contexts of the concept were learned and internalized.

A teacher who fails to engage a child about his/her life outside of school is doing that child a disservice and may delay her cognitive development. If the child's environment is not congruent with the teacher's presentation of environment, the child may become frustrated, feel inadequate, or at least misunderstand the concept. To be sure, if the child's family uses the word 'picnic' to describe sharing food with family and friends outdoors, the child will eventually develop his concept to include variants. Perhaps the potential confusion lasts a while and may cause further confusions as the child experiences more disjointed connections from the teacher. Vygotsky (1986) warned against engaging a child on things she is already capable of doing without assistance, and to confuse or frustrate a child. One will result in boredom, and the other, over time, is likely to cause a child to dislike school and learning. Further, according to Vygotsky (1986, p. 8), attention must be taken to allow concepts to develop through experiences and multi-modal communication because "certain thoughts cannot be communicated to children 
even if they are familiar with the necessary words." This principle supports child-directed curricula in preschools where children construct their knowledge.

There is no orderly prescription for teachers to follow to reach intersubjectivity with children, nor is there a defined list of expectations one can reference to know it is in process. Because of these realities, intersubjectivity was investigated in the current study via qualitative research following a phenomenological case study model. Teachers and their classes were chosen based on criteria of incongruent sub-culture representation between teacher and the majority of children. Instructional communications were videotaped to capture non-verbal as well as environmental evidence supporting intersubjectivity. Semi-structured interviews of teachers gathered evidence of teachers' awareness and understanding of intersubjectivity, even if the term is unknown.

I wondered whether identifying the process involved in reaching intersubjectivity would be elusive in preschool classrooms, no matter the subculture, or what educational level was reached by the teacher. To determine this, I developed indicators of interactive skillsets based on qualities of intersubjective communication, as well as indicators of success - that there is mutual understanding between preschool teacher and child that include observation, demonstration, open-ended questions and wonderments. 


\section{Figure 1}

\section{Conceptual Funnel of Guided Participation}

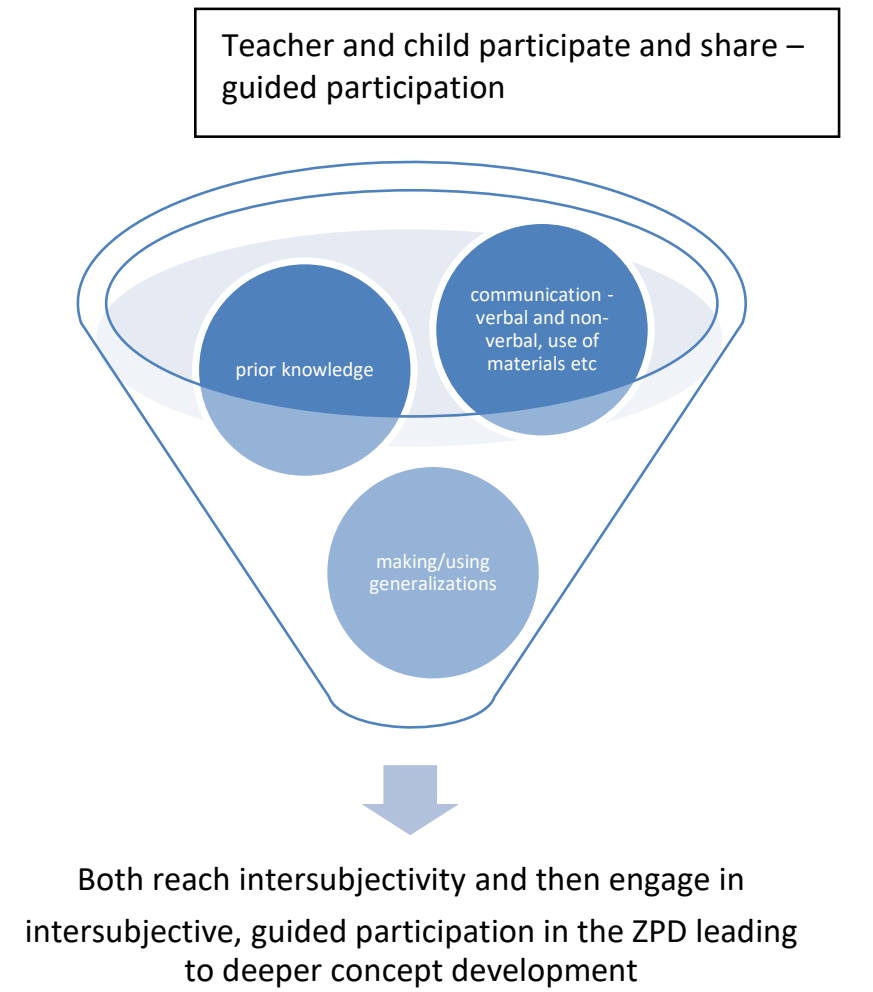

Figure 2

Process of Intersubjective Instruction

\begin{tabular}{l|l} 
PRIOR TO INSTRUCTION & ENTER THE ZPD \\
Guided participation $=$ & \\
intersubjective & \\
communication & That intangible place \\
GOAL = Assess prior & where a child can \\
concept knowledge & perform tasks with \\
help from MKO, not \\
yet independently
\end{tabular}

GUIDED PARTICIPATION

Instruction in the ZPD with the goal of next-level or deeper-level cognitive development.

Introducing concepts: Teacher demonstrates;

Teacher and child do together;

Child does on her own with verbal guidance, encouragement, support
MONITOR CHILD'S FRUSTRATION

If there is a little challenge and kind encouragement progresses the task, keep going until child works independently. [Children demonstrating higher degree of frustration should be kindly redirected. They are not ready - try another time]

\section{OVER TIME}

With experiential opportunities, the child will practice newly taught skills.

Teacher will use guided participation and verbal support as needed, then back away until child works independently, showing internalization of deeper concept knowledge 


\section{Theoretical Framework and Research Paradigm}

The theoretical framework was taken directly from Vygotsky's cultural-historical theory of cognitive research (sometimes written as socio-historical theory of cognitive development). Vygotsky's $(1978,1986,1997$ a) work made its way from Russia to the United States over forty years ago, and intrigued the fields of psychology, education, and sociology, to name three. Continuous study and research of his theory and publications resulted in an intensive acceptance of many elements of his cultural-historical theory of cognitive development; among them are the concept widely known as scaffolding, the zone of proximal development (ZPD), meaningful relationships, and culture as that which is passed down through generations in a social environment. It is Vygotsky (1978) who, in his research on thinking, described the concept that has come to be called intersubjectivity; that intersubjectivity is the critical precursor to effective scaffolding.

Berk and Winsler (1995) explained a key requirement to effective scaffolding children's knowledge - intersubjectivity, during which "two participants who begin a task with a different understanding arrive at a shared understanding. ... achieve true collaboration and to communicate effectively during joint activity" (p.27). Teachers who fail to consider the perspectives of children when engaging in joint activity cannot effectively scaffold learning.

According to Merriam (2009), qualitative, phenomenological studies strive to understand the whys and wherefores of phenomena, in this case, intersubjectivity. Interestingly, intersubjectivity is itself phenomenological, in that it seeks common understanding between two or more people (Thompson, 2005). Geertz (1973) inspired me to use the interpretivist paradigm in studying teacher-child engagement toward intersubjectivity. My main concern in conducting this study was to investigate engagement toward understanding between teachers and the 
children in their classroom. Meaning and understanding have cultural roots thus, attending to cultural congruency or incongruencies may have a bearing on the data to be collected.

Engagement to reach intersubjectivity is important, as is the culture behind the engagement. The dynamics of this study fit within the parameters of a phenomenological, interpretivist paradigm.

The art of pedagogy, unique to each individual teacher, influenced the decision to design this research using a case study format. A teacher's role is to differentiate instruction addressing individual children's backgrounds (cultural, linguistic, or ability diversity). Intersubjectivity is the phenomenological case, and each of the three teachers recruited was a means to study the case. This strategy was intended to illustrate communicative styles that lend themselves to intersubjectivity, and to create a pathway to discussing cultural differences or same-ness between teachers and the children in their classrooms. Designing this research for the investigation of intersubjectivity allowed in-depth descriptions of the unique, multi-variate qualities teachers bring to the process of communication. It is a human quality to seek communication with others as a process of cultural development; first a child engages socially (interpersonally), and then within himself, intra-personally (Vygotsky, 1978).

\section{Context of the Study}

The purpose of this study was to explore intersubjectivity in the context of intentional preschool teacher and child communication in early childhood environments. In semi-structured interviews, teachers discussed their interactions with children, the depth of their interactions, and whether they were cognizant of the need for both to mutually understand the other to know where they are going, ergo, mediating knowledge. A key factor in successful mediation of knowledge is a teacher's sensitivity to cultural differences between herself and the children. Where Vygotsky conducted his research on ethnic cultures, this study sought to extract evidence 
of each teacher's responsiveness to their children's background cultures by relating learning to the child's history. Cultural sensitivity and intersubjectivity are intertwined; to reach one is to reach the other because development is cultural (Vygotsky, 1978, 1986). For example, imagine a teacher talking to a small group of children about places they play at home. One child may respond, 'In my house.' The teacher may respond, 'Anywhere else? Outside?' If the child's sole response is 'In my house,' the teacher may inquire, 'Do you play in the yard?' using the concept of a 'back yard.' The child repeats, 'In my house.' A teacher who fails to inquire about the child's house is not being culturally responsive. If she was responsive there would be questions such as, 'Tell me about your house.' She may find that the child lives in an apartment with no yard, and in an unsafe neighborhood.

Chances are, according to the National Center for Education Statistics (2009), the teacher was not raised in a similar environment to the children in her class. The apartment-dwelling child may not have outside experiences in a back yard, or of going to the park. He may, however, have many weekend experiences with extended family away from the neighborhood. By investigating her student's life experiences, thus current conceptual knowledge, she will be more effective in talking about places children play. Teachers who do not make inquiries to better understand their students are apt to misjudge the capabilities of the children in their care. If intersubjectivity is not reached, without inquiry and 'serve and return' conversation (Serve \& Return Interaction Shapes Brain Circuitry, 2017), the teacher may assume the child understands her when she does not, resulting in ineffective instruction. Over time, this child may demonstrate challenging behaviors because she is not being understood, causing frustration.

Over many years of observing preschool teachers in the classroom and reading of Vygotsky’s (1978, 1986, 1997a) work in texts, a potential disconnect surfaced. As teachers 
endeavored to 'scaffold' learning in the ZPD, they tended to maintain teacher-directed instruction, and used close-ended questioning. The idea that "children learn in the context of meaningful relationships" (Shore, 1997, p. 33) is misinterpreted by many preschool teachers, as they continue to teach in the manner they see as appropriate, without getting to know the children, their interests, and how they perceive concepts. Understanding the concept of 'scaffolding' is also suspect, as many teachers I have worked with over the years seem to think it means 'teaching.' They forget or are unaware that knowledge is a continuous process of concept development.

As I progressed on my career path and observed more and more teachers, I noticed a persistent preference of teachers to engage heavily in direct instruction - where they tell children what they (the teacher) determine to be what the child should know and do - without making the effort to see if the child understands what is being taught. While direct instruction has its place (classroom rules, safe use of materials, for instance), this practice is, in large part, discouraged in early childhood high quality, progressive circles, and in college and university teacher education programs. Child-centered instruction is considered most effective in that new material is presented to children referencing their interests, which implies efforts to be intersubjective - to approach learning on the same plane of understanding.

To embark on a journey to understand whether preschool teachers, knowingly or unknowingly, engage children toward intersubjectivity, we need to investigate whether teachers feel it is important for children to understand what teachers are communicating, and vice versa. This approach warranted a phenomenological study in which teachers considered their experiences with children in their classrooms, and their philosophy of teaching. 


\section{Summary}

The purpose of this study was to explore intersubjectivity in the context of intentional preschool teacher and child communication in early childhood environments. Berk and Winsler (1995) and Abtahi et al. (2017) indicated that scaffolding knowledge in the ZPD is ineffective without MKOs and learners engaging toward intersubjectivity. Intersubjective communication is the process by which preschool teachers and children engage each other, using multi-modal methods, to reach mutual understanding. Do preschool teachers recognize the critical importance that children do not fully understand concepts based on verbal statements? This study sought to understand whether there is a presence of intersubjectivity in preschool classrooms. In the next chapter, I will review the literature, exploring concepts of intersubjectivity, scaffolding, and the zone of proximal development, as well as research to support the importance of reaching intersubjectivity prior to, and in the process of effective, scaffolded learning. Chapter 3 will discuss the methodology that was applied in this study. 


\section{CHAPTER II: LITERATURE REVIEW}

\section{Introduction}

Lev Semenovich Vygotsky was a Russian Jew whose short life (1896-1934) and work were greatly influenced by Tsarist rule, the Russian Revolution, Communism, and 14 years of tuberculosis, which took his life at age 37 (Gredler \& Shields, 2008; Kozulin, 1986; Van der Veer \& Valsiner, 1991). In spite of poor health and government scrutiny, Vygotsky was passionate about psychology, bringing backgrounds in many disciplines (including art, education, history, and literature) to his perspectives, experiments, and writing. With his health failing by degree and over time (Van der Veer \& Valsiner, 1991), one might understand that Vygotsky documented as much of his work as possible without the luxury of time for organization. In fact, some of his work was published as much as 50 years after his death, and much is still unpublished (Van der Veer \& Valsiner, 1991).

Perhaps the expansive scope of his work, the fact that his translated written works included repetitions, discussions without contemporary relevance, and muddy points (at least to Western readers), and the absence of Vygotsky voice for rebuttals and confirmations caused his work to be "constructed, deconstructed, and reconstructed" (Glick, 1997, p. xi). In the process, concepts can get lost or misconstrued. Vygotsky detailed his concept of the zone of proximal development, within which learners are nudged past their current level of demonstrated, independent competence to a more complex level of understanding and/or doing; one their MKO determines the learner can do and does not yet realize. Wood et al. (1976) introduced us to the term 'scaffolding' to further explain Vygotsky's concept of learning in the ZPD, using a tutorial format to describe the interactions Vygotsky (1986) sometimes called 'mediated understanding.' This phrase, along with others used to explain the concept, evolved into what Trevarthen (2008) 
coined in 1974 as 'intersubjectivity,' also described as common, or mutual understanding. Now we have additional terms for the dynamic Vygotsky holistically described as the ZPD, where learning happens. The literature review of scaffolding will indicate a muddying, dissection, or dilution of what Vygotsky $(1978,1986,1997 b)$ considered learning.

This review of literature funneled Vygotsky's cultural-historical (also referred to as socio-cultural) theory of cognitive development into the zone of proximal development (ZPD), the concept largely referred to as 'scaffolding,' and then, to intersubjectivity, and its importance in the process of concept development. The theory itself is important for teachers to recognize the cultural bases of cognitive development. Next, I presented reviews on the ZPD, the term defined by Vygotsky, and within which cognitive development happens. Following this is a discussion surrounding scaffolding as a metaphor to describe the concept written about by Vygotsky that illustrates the potential for misunderstanding teachers may experience when using the strategy to teach young children. Then, discussion on mediated activity and intersubjectivity is presented. This term is also neo-Vygotskian, although there is a great deal written that clarifies and expands his descriptions with continued research surrounding its importance. This chapter focused on work related to preschoolers.

My first reference to the term intersubjectivity was from Berk and Winsler (1995), where I constructed the following understanding: that teachers must be consistent and intentional to engage children (learners) toward intersubjectivity in order to scaffold their learning in the ZPD. Searches using keywords ZPD and scaffolding generated a plethora of references. However, the term intersubjectivity proved a challenge in education databases. Widening the search net, removing all filters, yielded references to intersubjectivity in many disciplines including anthropology, philosophy, and language and communication, all areas of interest to Vygotsky 
(Kozulin, 1986). My research was to explore intersubjectivity as it referred to teacher-child interactions during learning in the ZPD. Therefore, my search into the dynamic focused on research in language and communication. The summary at the end of Chapter 2 brings clarity to the funnel: Cultural-historical theory of cognitive development, ZPD, concept development and scaffolding, and intersubjectivity.

\section{Problem Statement}

As indicated by Glick (1997, p. xi), scholars have been "constructing, deconstructing, and reconstructing Vygotsky's work since it came to the attention of the West. The impact of his cultural-historical theory of cognitive development brought his concepts deeply into the fabric of American educational and developmental standards including the Illinois Early Learning and Development Standards (isbe.net), Illinois Professional Teaching Standards (ISBE, 2013) and NAEYC Professional Development Standards (naeyc.org). And yet, the deconstruction and reconstruction - especially of Vygotsky's $(1978,1986)$ written work on the ZPD, and instruction within it - may have generated misunderstandings among teachers regarding interactive communications critical to the establishment of intersubjective perspectives and instruction in the zone of proximal development.

\section{Vygotsky's Cultural-Historical Theory of Cognitive Development}

Vygotsky's $(1978,1986,1997$ a) work made its way from Russia to the United States over forty years ago, and intrigued the fields of psychology, education, and sociology, to name three. Continuous study and research of his theory and publications resulted in an intensive acceptance of many elements of his cultural-historical theory of cognitive development; among them are the ZPD and instructing with the zone, the concept greatly known as scaffolding, and culture as that which is passed down through generations in a social environment. 
If we think of a basic nature/nurture simile, Vygotsky $(1978,1986,1997$ a) saw child development along two planes: first, biological, then historical (cultural). Biology was considered in the context of genetics, growth, and maturation, and history as the culture into which a child is born (Van der Veer \& Valsiner, 1991; Vygotsky, 1978; Wertsch, 1985), and over which, according to Vygotsky (1978), the child has no control. He posited that elementary psychological processes "are of biological origin, and the higher psychological functions, of sociocultural origin. The history of child behavior is born from the interweaving of these two lines" (Vygotsky, 1978, p.46). His cultural-historical theory, therefore, casts a wide net over biology and culture as it relates to cognitive development. "At the heart of Vygotsky's theory lies the understanding of human cognition and learning as a social and cultural, rather than individual phenomena" (Kozulin et al., 2003, p. 1).

Cognition and learning as social phenomena are not the end of this theoretical story. The complexities of higher mental functions recognize the social process, and the individual function of internalization as well as self-regulation (Karpov, 2003; Mahn, 2003; Van der Veer \& Valsiner, 1991; Vygotsky, 1978, 1986, 1997a, 1997b; Wertsch, 1985). Vygotsky used four principles to differentiate higher mental functions:

(1) the shift of control from environment to the individual, that is, the emergence of voluntary regulation; (2) the emergence of conscious realization of mental processes; (3) the social origins and social nature of higher mental functions; and (4) the use of signs to mediate higher mental functions (Wertsch, 1985, p. 25).

There is a point in young children's lives, between one and two years of age, at which they experience an epiphany at the recognition that verbal signs (words) have meaning (Vygotsky, 1978). Objects (tools) are given names that, until then, had little or no meaning. In 
his account of human development, Vygotsky (1978) wrote in italics, "the most significant moment in the course of intellectual development, which gives birth to the purely human forms of practical and abstract intelligence, occurs when speech and practical activity, two previously completely independent lines of development, converge." What we term today as active learning, Vygotsky (1978) termed 'mediated activity.' In contrast to his contemporary constructivist Jean Piaget, Vygotsky stressed language as critical to learning (Daniels, 2001; Kozulin, 2003; Portes \& Vadeboncoeur, 2003; Vygotsky, 1978, 1986; Wertsch, 1985).

\section{The Zone of Proximal Development}

This is where, according to Vygotsky $(1978,1986)$, social interaction with more learned others, jointly engaging in mediated activity, leads to learning. Defining the zone of proximal development (ZPD) another way, it is the distance between what a child can do without assistance, and what she can do with the assistance of an MKO (Vygotsky, 1978, 1986). The ZPD is the core of Vygotsky's discussions about learning one's culture, learning via interaction between MKO's and children, using language materials and actions to further develop toward higher mental functions. The interactive, i.e., mediated activities using tools and symbols, is the process Wood et al (1976) named 'scaffolding.' Vygotsky refers to this process as pedagogical psychology (Vygotsky, 1978, 1986, 1997a, 1997b).

Vygotsky $(1978,1986)$ determined that true learning is accomplished with the assistance of MKOs, involving much more than imitation, attention, or memory. It is here, in the ZPD, where the child is reliant on MKO's use of intersubjective discourse and demonstration, setting the stage for the child to mediate her own concept development. It is a child's own mediation of concepts (problem solving), reliant on many modes of communication (e.g., words, ideas/examples, gestures) provided by a teacher or MKO. Children put themselves in the ZPD 
when they engage in pretend play. According to Vygotsky, pretend play in the ZPD is "A vital transitional stage toward operating with meanings as with objects," and through which "the child achieves a functional definition of concepts or objects, and words become parts of a thing” (Vygotsky, 1978, p. 99). In a preschool setting, this implies that much can be learned in all domains of development (physical, cognitive, social, and emotional) through pretend, and dramatic play, due to young children's propensity to use a home setting as the stage for such activity.

Vygotsky (1978) believed "Learning and development are interrelated from the child's very first day of life" (p. 84), and that children develop in a holistic manner rather than in stages. Vygotsky presented an innovative view that, while children need to mature in some ways to learn, he made a case for learning leading development (Berk \& Winsler, 1995; Vygotsky, 1978, 1986). He contends that there is greater value to consider a child's cognitive development based on their potential rather than their current ability (Vygotsky, 1978, 1986), which speaks to the importance of teachers acting as mediators in the development of knowledge. For example, a child that is trying to complete a 24-piece cardboard puzzle is shown strategies and supported through the task, enabling the successful completion of the puzzle. If the mediation was congruent with the child's potential level of development, the child will be able to practice putting the puzzle together with little (and eventually no) assistance. Once the child has learned to complete the puzzle without assistance, this particular behavior raises her ZPD to a more complex level, perhaps to 48-piece puzzles. If, however, on the next day, this same child shows great struggle completing the same puzzle, the teacher must consider whether her communication during guided participation with the child was effective to scaffold her learning to complete the task (Kozulin et al., 2003; Vygotsky, 1978, 1986). The teacher will have to make 
additional efforts to engage the child in multi-modal communication to arrive at mutual understanding.

The ZPD is that unseen place where, for those of us who subscribe to the sociocultural theory of cognitive development, learning happens; concept development progresses (Berk \& Winsler, 1995; Vygotsky, 1978, 1986) individually, through social, pretend play, with peers, and with MKO's that include teachers. Concept development through mediated activity and scaffolding, and intersubjectivity are phenomena in which teachers and children actively engage in the ZPD. In this chapter, each area is discussed separately, with a conclusion to synthesize their importance to early childhood development.

\section{Concept Development}

The definition of 'concept,' according to English Oxford Living Dictionary (2017), is “an abstract idea." The definition of 'idea' is "A concept or mental impression," and also, "A thought or suggestion as to a possible course of action." For example, 'I don't think that is a good idea.' Concept, and idea are synonyms. Concept development is cognitive development is learning.

Now comes the discussion of concept building as a process. According to Vygotsky (Kozulin, 2003), reflexologists had the wrong impression in the belief that human reflexes offered a valid connection to the construction of behavior. He did, however, yield to the premise that "reflexes provide the foundation of behavior" (Kozulin, 2003). I feel that Vygotsky would have come to change his perspective had his health and relationship with the Soviet government allowed. While I agree that reflexes are the foundation of behavior, I also contend that reflexes (perhaps more than others) generate social interaction. For example (and given a healthy environment), a newborn's cries will be answered with mother attending to the baby. She will show a pleasant face, speak in a soft and tonal voice, and will then change and hold, pick up and 
feed while holding, pick up the child, or perhaps cover/swaddle the child. After a period of time, the child, in my opinion, will come to expect mother or another significant person to appear when she cries. When all goes well, the mother will come to learn the baby's cries and interpret whether she is hungry, needs a diaper change, is cold, or wants to be with others. Further, and in all these scenarios, mother should talk with the baby, for example, "Are you hungry? Shall we get ready to eat? Here's your milk." Mother is putting words to the actions, and child is hearing everything, and storing it away. Vygotsky says that learning is socially mediated (Vygotsky, 1978, 1986) and I believe a baby’s cries elicit social interaction. Cries as a mode of communication develop into cooing, babbling, two-word utterances, and so on to full language acquisition (Pence Turnbull \& Justice, 2017). My work, however, was not intended to contradict Vygotsky's viewpoint on reflexes and learning, rather it was important to understand Vygotsky's perspectives on concept development and apply them to learning in preschool.

It was through the observations of children who we consider of preschool age that Vygotsky concluded that both "speech and action are one and the same complex psychological function, directed toward the solution of the problem at hand" (Vygotsky, 1978, p. 25). Further, as a result of many observations, they "lead me to the conclusion that children solve practical tasks with the help of their speech, as well as their eyes and hands” (Vygotsky, 1978, p. 26). As to when this behavior becomes more apparent, Vygotsky points out that egocentric speech wanes as children come to realize they cannot solve problems my themselves, seek adult intervention, and verbalize the solution being sought (Vygotsky, 1978). For example, there is a point, usually through toddlerhood, when children are very possessive of things. Perhaps a parent notes that a toy has some function such as a door opening once a lever is pulled. If the parent intervenes with the child, she may protest with facial expression, and speech such as "No! It's mine!" This child 
is not at a point of seeking a solution to that particular problem. Another example may be a child who, feeling hungry, seeks Daddy to get a favorite cereal from the shelf and says, "Daddy, please get the cereal for me?" The child may even ask to be picked up to get it herself. Here we have action with words. Continued experiences, along with verbalizations offer a child many ways in which to understand the meaning of words and behaviors. In Vygotsky's use of the word language, however, there is more to communicating than words.

As children grow, their experiences should be rich, social, varied, and include interaction and dialogue. As new words and concepts are introduced, it is imperative that these words and concepts be repeated, and also presented in various ways. Before concepts are truly generalized (a broad experience of the concept that can be expressed verbally), children categorize objects based on attributes that may or may not be representative of a true concept. This is due in part to maturity and in part to sociocultural experiences that include cooperative interaction (Vygotsky, 1986). For example, a young child may see a red object and a teacher labels the item red. To be sure the child starts to recognize that 'red' is a color rather than the object itself, there needs to be reference to other things that are red. However, a second, third, or fourth object may well have a different shade or hue of red, resulting in the child's understanding of 'red' to mean that many different shades of the color. As her experiences grow, and teachers and parents engage the child in language, the child will come to know that each item is not simply red; the color red can be manipulated in many ways to change its shade and hue. Until that concept is fully understood, Vygotsky believed that a concept of red is a complex generalization and not a fully developed, understood concept (Vygotsky, 1986). The discussion about 'red' is an example that "word meanings are dynamic rather than static formations. They change as the child develops; they change also with the various ways in which thought functions" (Vygotsky, 1986, p. 217). 
Non-verbal communication such as expressions, movements, and gestures are an important component of concept development to which Vygotsky only partially subscribed. He admitted that he would use an expressive gesture to help convey the feeling of 'cold,' for example, yet holds to the tenet that only through generalization and conceptual designation, will an interlocutor fully understand. Vygotsky goes on, "That is why certain thoughts cannot be communicated to children even if they are familiar with the necessary words. The adequately generalized concept that alone ensures full understanding may still be lacking” (Vygotsky, 1986, p. 8). In the years since Vygotsky's work came to the West, there has been much study on language and communication in general with attention paid to non-verbal components. The interaction that was explained somewhat in his writing - the need for children to be engaged in language with a teacher/parent along with activity in order to develop concepts - has been expanded greatly, and given the term 'intersubjectivity', to be discussed further along in the literature review.

According to Vygotsky, young children learn words (symbols) for behaviors, tasks, and things. However, it is only with practice of context using learning materials (tools) and/or symbols that meaning of words and concepts begin to develop. Eventually, words turn into tools as abstract representations (Vygotsky, 1978, 1986). Concept development, according to Vygotsky $(1978,1986)$ is accomplished through intersubjective guided participation/mediated activity (also defined as scaffolding) in the zone of proximal development.

\section{Guided Participation / Mediated Activity}

Scaffolding was not a term used by Vygotsky. Conversely, it illustrates the next generation of researchers and thinkers of child development, cognitive development, psychology, language development, education, and related disciplines, also known as neo-Vygotskians. In a 
study conducted at Harvard's Center for Cognitive Studies, scaffolding was introduced as a method of tutoring. Children who were three, four, and five years of age were guided in completing a three-dimensional pyramid of interconnecting blocks; a task that would be considered beyond their independent capabilities (Wood et al., 1976).

The results indicated some of the properties of an interactive system of exchange in which the tutor operates with an implicit theory of the learner's acts in order to recruit his attention, reduced degrees of freedom in the task to manageable limits, maintains "direction" in the problem solving, marks critical features, controls frustration and demonstrates solutions when the learner can recognize them (Wood et al., 1976, p. 99).

In this study, Wood et al. (1976) took care to consider frustration levels of the young children, and to break the task up into manageable segments. Gail Ross (Wood et al., 1976) is credited for her disposition in getting children to complete tasks to the best of their proximal abilities. However, the data involving interaction separated verbal telling, correcting, or reminding from actual demonstration. Further, the environment in which the study was described as "the experimental room... with a small table and 21 blocks of varying sizes spread out in a jumble" (Wood et al., 1976, p. 92). Vygotsky's writings indicated that children learn best when action and words are presented together, in a meaningful context, and certainly for these preschoolers, in a play-based environment. In addition, there is no evidence that the tutor engaged in discourse to assess the children's current level of conceptual development (Vygotsky, 1978, 1986).

Neo-Vygotskians Bodrova and Leong (2007) established an approach for educators in early childhood and primary grades. Styled after the work done by post-Vygotskian scholars in Russia, it is applied in Tools of the Mind schools in ten states, including Illinois. Regarding the 
concept of scaffolding, Bodrova and Leong (2007) shared three ideas: (a) levels of teacher assistance vary with each child, yet never to make a task easier; (b) as the child grasps the behaviors being scaffolded, responsibility for performance switches over to the child; and (c) support and assistance to the child is temporary and recedes slowly, facilitating independent problem solving. Another example of scaffolding comes from a qualitative study exploring teacher-child relationships in a mixed-age (three to five years) early childhood classroom (Fumoto, 2011):

In Nancy's class, they often look at their individual folders, with photographs of their experiences at the school and at home. Nancy calls each child and shares experiences, which often stimulate conversation:

Nancy: Hey Kevin, would you like to see your pictures? [As Kevin walks towards her, she asks him to go and get his folder saying, 'it starts with ' $\mathrm{K}$ '. They look at the folder together and talk about what he did when he first joined her class and then turn the page.]

Nancy: Kevin and cicada! I looked up the internet [sic] to see what it eats. [Kevin becomes animated and talks about the cicada he had found. Richard who was standing nearby joins in and the conversation turns to insects that they saw.] (p. 27)

A brief synthesis of this interaction to the Vygotsky theoretical concepts discussed in this review begins with the notation that children often look at their individual folders containing photos from home and school. Looking at this child's growing historical record offers the opportunity to continue developing concepts such as past and present. As information is added to this record, the child's past grows, which is a development of the concept 'past.' The teacher, 
Nancy notes that his folder starts with 'K,' and makes no further mention. My thought is that Nancy knows Kevin is able to identify ' $\mathrm{K}$ ' correctly and makes the comment for reference and reinforcement. This is an important detail in pedagogy, speaking to Zaporozhets' concept of "amplification" of development; the need for "expansion and enrichment of content of appropriate activities rather than acceleration into inappropriate activities" (as cited in Bodrova \& Leong, 2007). In this instance, Kevin will see that the reference that his folder starts with ' $\mathrm{K}$ ' is about alphabetical order as well as the first letter of his name. Nancy's enthusiasm about Kevin and the cicada (interpreted from the exclamation point) opened the door for him to talk about it, which he did. Concurrently, teacher called up information on cicadas on the internet, extending a personal past experience to the present and an expansion of content. In addition, classmate Richard overheard the conversation and joined in further expanding the conversation to other insects they saw. This is social learning that is meaningful and interesting to the children. The teacher did not impose this information. She acted as human mediator as well as an active participant in concept building, allowing both children to mediate their own learning.

\section{Intersubjectivity}

Continued development of meaning relies on communication between teacher and child in the form of sharing perspectives and ideas using more than verbal dialogue. Non-verbal communication is critical to a young child's development of understanding and can be found in the form of facial expression, intonation, gestures, demonstrations, movement, etc., repeated over time and in different formats, to enhance understanding, which equates to cognitive development (Vygotsky, 1978, 1986).

To explore the dynamics of intersubjectivity it is important to revisit concept development. According to Vygotsky (1986), concepts are not fully and completely understood 
in early childhood. At the beginning of the process, very young children are given verbal labels to objects, and they amass a cache of labels, Mommy Daddy, cat, dog, car, for example. With more experiences, attention and memory development, joint referencing (Kozulin et al., 2003; Rochat et al., 2009; van der Veer \& Valsiner, 1991), and cooperative interaction (Kozulin et al., 2003; Rochat et al., 2009; Vygotsky, 1986), children will start to make connections between objects based on attributes of the child' design. These chains of object words are referred to as 'complexes' by Vygotsky (1986). He warns that the connections may not be correct yet, which is why continued interactions with MKOs, acting as human mediators, will help children toward true understanding of concepts.

Before reaching that level of understanding, however, Vygotsky's studies suggested that pseudoconcepts are constructed in early childhood (including preschool-aged children), based on "very concrete perceptual features" (van der Veer \& Valsiner, 1991, p.111). For example, a child may pick out a triangle from many geometric blocks upon request. The child with developed pseudoconcepts will be able to choose a triangle no matter the color, size, or material. The perceptual features of three straight sides are understood. Past these concrete perceptual features, it is questionable whether a preschooler can verbally define and abstractly understand 'triangle' as a polygon or a closed plane figure having three sides and three angles. And then, there are more applications of the concept, 'triangle,' such as the instrument, or the tool used to set up pool balls.

Facilitating the development of concepts requires adult (MKO)-child interactions. As mentioned earlier, the responsibility of concept/cognitive development relies not only on interactions; it relies on the "expansion and enrichment of content of appropriate activities rather than acceleration into inappropriate activities" (Zaporozhets as cited in Bodrova \& Leong, 2005). 
Before this concept-building can commence, Vygotsky also indicated that adults must ascertain where the children are in their development to proceed from that point to scaffolding. Teachers that verbally impose their knowledge on children without seeking representational understanding are not effective in scaffolding learning.

Intersubjectivity, communication working toward common understanding between two or more interlocutors must, therefore, be mediated prior to scaffolding learning. The function, however, is not to reach complete understanding, as indicated by Newman, Griffin, \& Cole (1989):

Just as the children do not have to know the full cultural analysis of a tool to begin using it [a ruler, for example], the teacher does not have to have a complete analysis of the children's understanding of the situation to start using their actions in the larger system [being constructed]. (p. 63)

Through intersubjectivity, the child is exposed to the teacher's understanding, and the teacher is exposed to the child's understanding. Rogoff (2003) referred to intersubjectivity as a mutual bridging of meanings. Indeed, the teacher must be intentional in striving to understand how and what the child understands in order to expand and enrich the concept/problem at hand. Again, teachers must remember that a concept is not yet understood to its fullest extent by the children, even though children may apply words correctly (Rogoff, 2003; van der Veer \& Valsiner, 1991). "Concept formation is the result of such a complex activity, in which all basic intellectual functions take part [memory, attention etc.].......all these are insufficient without the use of a sign or word. Words and other signs are those means that direct our mental operations, control their course, and channel them toward the solution of the problem.... (Vygotsky, 1986, pp. 106-107). 
Mediating intersubjectivity prior to scaffolding is important, and yet that is certainly not the end of things. Teachers should not engage toward intersubjectivity and then proceed with direct instruction or leave the child to his own devices of exploration. That contradicts the function of cognitive development as discussed earlier. Intersubjectivity must be a constant, intentional effort of every teacher at all times; to expand a child's verbal capabilities, to develop a child's representations of concepts, and toward concept development. "Intersubjectivity is based upon participation in joint action.... Participatory engagement with signifying objects accompanies and underpins the child's entry into the symbolic realm of language" (Sinha \& Rodriguez, 2008).

The culture of the United States, an aggregate of many cultures, is driven by communication in the form of written and verbal language. Developmentally, children learn verbal language before written language (Berger, 2018). To understand each other, and to share our culture(s), we must communicate our beliefs and behaviors in such a way as to allow both parties to adjust to the other's perspective (Berk \& Winsler, 1995; Rogoff, 2003; Sinha \& Rodrigues, 2008). In this process, we must also recognize the function of non-verbal language as we communicate. Intersubjectivity is a social activity, and social activities involve emotional as well as cognitive sharing (Rogoff, 1990, 2003; Vygotsky, 1978, 1986). Gestures, facial expressions, tonal voice, pauses, movements, guidance, and demonstrations fall under non-verbal communication.

Examples are in order if I am to be intersubjective about sharing this concept with readers. Consider an infant's steady attention as a parent, one face close to the other, presenting a loving expression with eyes widened, smiling, and speaking softly with a higher pitch than usual. Emotion is being exchanged, and the infant will likely show facial expression. A little later, 
when the infant smiles, it often includes a smile with widened eyes. Again, emotion is being exchanged, suggesting the eagerness of infants to be socially connected. Later infancy and toddlerhood find children pointing and verbalizing to call adult attention to an object, and vice versa. Joint attention is considered a child's way of learning about something, or perhaps to indicate a desire for an object (Berger, 2018; Gallagher \& Hutto, 2008; Krichevets, 2014; Trevarthen \& Aitken, 2001). The growing study of intersubjectivity in disciplines including cognitive psychology, developmental psychology, and language development categorize levels of intersubjectivity based on the level of participation of both child and adult (Krichevets, 2014; Trevarthen \& Aitken, 2001). Therefore, this review was focused on intersubjectivity as it relates to preschoolers. One of the greatest tools a preschool teacher can employ is a good open-ended question. Here is a short conversational transcript from a qualitative study done in Norway (Gjems, 2013). The teacher was sitting at a small table with three girls, all three years old, playing with plastic blocks:

Pia (3) introduces the conversational subject with the following declaration:

1PIA: I live in Norway - ! [=aloud, quick]

2TEA: yes - you live in 〈Norway $\rangle_{-}$? [=wondering tone, looks seriously at Pia] 3PIA: there ain't any lions in Norway - ?! [=quick, both asking and ascertaining] 4TEA: no - why not, do you think - ? [=smiles at Pia] 5INE: no, there are no lions in Norway - ! [=talks aloud and fast 6INE: and by the way - [=seems scared and have a haunted look] 7INE: tigers, they only live in Africa - ? [=looks serious at the teacher] 8TEA: oh, yes, they do - ! [=nods and smiles] 
9PIA: and they don't come here - ? [=looks at the teacher with a begging expression in her face]

10TEA: [=shakes her head]

11PIA: bears and foxes -

12PIA: they are dangerous - ! [=talks aloud, haunted look]

13PIA: if we want see them -

14PIA: we have to drive far away - ? [=looks serious at the teacher]

15TEA: oh, yes - drive far away -

(The conversation continues and they talk about small Norwegian animals and where they live.) The 'problem' in this conversation was when to be afraid, according to the author. By stating her home, Norway, and clarifying that lions and tigers, bears and foxes live far away, it is suggested that Pia can rest assured that she will not have to fear contact with any of those 'dangerous' animals. I wonder if the teacher presented facial expression that was not documented, such as at her last comment, to offer emotional comfort to Pia.

Note that one of the children did not contribute to the conversation. There are many possibilities when considering the quiet child; she may have a different attitude toward lions and tigers, and was considering Pia's behaviors (both verbal and non-verbal) as well as the others' reactions and input. Whatever the reason, the teacher may have asked the third child's opinion to see whether she shared similar views.

The literature review was concluded with an evaluation of a college-level curriculum text used in teacher education programs throughout the country, and how concepts of sociocultural theory are embedded in its content. I referred to the index of a well-regarded curriculum text (Kostelnik et al., 2015) that I used in my college-level curriculum class and looked up 
intersubjectivity, to find it was not listed. Moving to scaffolding, I uncovered a few references in the 537-page book. The first reference generalized scaffolding as a four-step process, leaving out any mention of true dialogue. The written narrative used an example of teacher holding a child's hand with items in it and, using her finger, point to each item and count one-to-one correspondence. It was very clinical, just as the more detailed examples of scaffolding found on page 59, again using this four-step process:

- I do...you watch

- I do...you help

- You do...I help

- You do...I watch (p.58)

A section on "scaffolding children's emergent literacy" (Kostelnik et al., 2015) discussed good, appropriate information on emergent literacy, yet made no reference to the four-step process indicated above, and there was nothing about enhancing concepts or dialogue with active learning. "For adults to be most effective at scaffolding literacy tasks with young children, they must have expertise with respect to child development and knowledge about the sequence in which literacy skills emerge" (Kostelnik et al., 2015, p. 371). Unfortunately, this was not an accurate way to express effective scaffolding. Scaffolding received a small mention in the chapter on the affective domain; that which referred to a child's emerging knowledge of their own emotions, the emotions of others, their self-concept, and self-regulation. Affective domain teaching strategy number 15 recommended the teacher "use scaffolding techniques to challenge children to perform tasks slightly beyond what they can easily do on their own" (Kostelnik et al., 2015, p. 320). This was actually a good partial definition of the ZPD and was followed by a list of eleven "appropriate" comments of praise to encourage efforts. Again, no interaction, context, 
or activity was suggested. The two comments listed illustrate first, over the top with judgmental tones and second, praise that was non-specific (Kohn, 1993).

- Now that's what I call a terrific job. You've remembered to leave spaces between every single word.

- That's really an improvement!

Next, and still in Kostelnik et al., 2015, I sought the word concept in the index, settling on a page indicating a definition. The definition itself was congruent with cognitive development descriptors related in this paper. After the definition I saw narrative that puts value on connections between concepts and thematic teaching, which was very limiting and did not address those limitations. The statements were, "The natural process of mentally connecting bits of information into more integrated ideas is enhanced through children's involvement in thematic instruction," and "teachers are increasingly interested in helping young children make conceptual connections through an integrated curriculum such as that used in thematic teaching” (p. 512). All too often, thematic units constructed antithesis to Vygotsky's own pedagogical thinking. I offer observations from my current and 20 year experience in early childhood settings in two states: (a) thematic units are often planned without input from their children, and traditionally last one week; (b) their 'concepts' are narrow in scope and teacher-directed, such as teddy bear picnic done in March before the weather cooperates with the developing concept of picnic; (c) integrating the concept throughout classroom activity areas does little to enhance understanding of the concept, as the comprehensive process of scaffolding is lost in translation. Teddy bear picnic will surface again next year with another group of children. 


\section{Summary}

Education and the highest forms of mental functioning are valued in our culture, involving the ability to understand concepts fully and abstractly. Vygotsky $(1978,1986)$ indicated that concept building (cognitive development) at its highest level is complete abstract understanding of concepts, and abstractions are made from verbal representations learned resulting from shared negotiation of understanding and activity. Until then and beginning at birth, adults engage children in social, joint interactions using verbal and non-verbal methods of communication as well as objects/tools to actively engage in concept building. Very young children learn the signs (words) for things such as Mom, Dad, dog, and car; words that represent a child's cultural background. A growing child will have more and more experiences, and parents/MKOs will put labels into limited meaningful context, adding to the child's developing understanding of concepts. More and more experiences, various contexts, and a growing lexicon, added to the dynamic of joint reference/cooperative interactions (both verbal and non-verbal) during active learning, indicate a child's development of knowledge (Kozulin et al., 2003; van der Veer \& Valsiner, 1991; Vygotsky, 1986).

Scaffolding is a metaphor used to explain guided participation through the zone of proximal development (Berk \& Winsler, 1995; Newman et al., 1989; Rogoff, 1990, 2003). MKOs communicate an expanded or new solution to a problem, actively engaging the child and enriching her cognitive development. Such communication is based on a negotiated understanding of the topic shared by the adult and child; negotiated through communication and activity. Intersubjectivity is "the process whereby two participants who begin a task with different understanding arrive at a shared [not same] understanding” (Berk \& Winsler, 1995, p. 27). Note that I consider guided participation the process and intersubjectivity the goal. 
Learning, according to Vygotsky $(1978,1986)$ brings new information to the current information a child holds about a concept. It occurs in the zone of proximal development, ZPD, which is a span in development between which a child can solve problems with the assistance of a teacher, and independently (Berk \& Winsler, 1995; Bodrova \& Leong, 2007; Rogoff, 1990; Vygotsky, 1978, 1986). Based on this collective information and thinking holistically, children would thrive in a language, social, and activity-rich environment where interaction is meaningful and responsive to emotional and cultural aspects of learning. It is through an understanding of Vygotsky's sociocultural theory of cognitive development, developing concepts by engaging in mediated activity (guided participation) in the zone of proximal development using verbal as well as non-verbal communication that informed this study.

This literature review illuminated the need for in-depth investigation of intersubjectivity; whether it is sought between teacher and child, whether it drives a teacher in her instruction of concept development through interaction, and whether it sustains instruction through guided participation. Since teacher-child interactions are unique to those involved, socially and culturally, the interpretivist paradigm employing case study research was used. Three case studies were conducted to investigate the phenomenon of intersubjectivity in preschool classrooms. 


\section{CHAPTER III: METHODOLOGY}

\section{Introduction}

Whether early childhood teachers take a training workshop, college-level courses, or read articles, they will, at some point, be exposed to scaffolding and the zone of proximal development (ZPD). Vygotsky's $(1978,1986)$ sociocultural theory of cognitive development is deeply embedded in education standards, professional standards, texts, and journals throughout the country. Professional development trainings are offered with related material on scaffolding or teacher-child relationships. Practitioners attend mandatory, professional development trainings and/or take early childhood courses that inspire them to exercise different instructional and behavioral strategies, methods, and activities in their classroom. Many take their newfound competence (which may or may not be fully understood) to students the next school day. What tends to happen, however, is that a teacher may exercise fragments of an approach or curriculum without benefit or understanding of the entire vision. This greatly compromises the quality of her professionalism (Buysse et al., 2009).

Drawing from my personal history as an early childhood educator, director, coordinator, and college faculty member, early childhood teachers are still enmeshed in teacher-directed pedagogy. Vygotsky $(1978,1986)$ recognized that there are certainly instances that warrant teacher-directed instruction, yet not at all to the degree that I have witnessed over the years. Teacher education is considered a contributing factor in teacher quality that includes more childdirected instruction (McMullen \& Alat, 2002; Early et al., 2007).

\section{Problem Statement}

One critical term, crucial to concept development in the zone of proximal development appears to elude discussion in textbooks; that being intersubjectivity, and how it is to be reached. 
There is great potential that many early childhood teacher education textbook authors misunderstand, thus misinform, teacher candidates about the terms attributed to Vygotsky's sociocultural theory. Illustrating the many iterations of the process toward reaching intersubjectivity, Rogoff $(1990,2003)$ used the term guided participation, which more closely resembles 'scaffolding;' Kostelnik et al., 2015, grouped intersubjectivity within a section of discussion on scaffolding, yet did not distinguish between the terms.

Vygotsky $(1978,1986)$ separated the concepts of instruction as occurring after common/shared understanding has been reached, further noting that during instruction, teachers must use effective communication to assure shared understanding is maintained throughout instruction. Without understanding there is no effective instruction. Gjems (2010) used the term negotiate meaning; Berger (2018) referred to intersubjectivity as joint engagement; and the term joint reference was found in work by Kozulin et al. (2003) and van der Veer and Valsiner (1991). Kozulin et al. (2003) also used cooperative interaction as a means to reach intersubjectivity, as did Vygotsky (1986); Bodrova and Leong (2007) used socially shared cognition; Newman et al. (1989) referred to everything within the ZPD as the construction zone; and Bruner (1990) referred to the concept development process as meaning making. Cognitive development scholars such as Rogoff $(1990,2003)$ and many language and communication scholars (Brinck, 2008; Trevarthen, 2008; Trevarthen \& Aitken, 2001; and Zlatev et al., 2008) consistently used the term intersubjectivity. Surely, with these many terms used for almost-thesame concept described by Vygotsky $(1978,1986)$ so many years ago, there is going to be confusion and misunderstanding. Terminology poses a problem for early childhood teacher education and professional development instructors. Indeed, it bears repeating that there is looping cause and effect before and during teaching; effective communication/instruction leads 
to intersubjectivity, facilitating concept development - learning and application of knowledge.

A number of persistent questions related to intersubjectivity exist, including, "What does intersubjectivity look like in preschool classrooms?" "Is there evidence of teachers engaging children toward mutual understanding?" and, "How do teachers and children enhance communication using non-verbal means?" It is also important to recognize whether preschool teachers are intentional about engaging children intersubjectively to find out, "Is teacher communication multi-modal, utilizing expression, tone, volume, gestures, and body language?" and, "How do the children respond to her instruction?"

As Vygotsky and his successors pointed out (Vygotsky, 1978, 1986; Zlatev et al., 2008), intersubjectivity is a crucial element of mutual understanding between MKO and learner (teacher and child, or between peers). A teacher must engage as the child in active and verbal communication to learn what the child is thinking. Only then, is the teacher in a position to engage in mediated activity to develop concepts. For example, discussing concepts such as home and family hold a wide variability of understanding for children. A teacher who fails to actively engage and listen to a child about his/her family will lose perspective of what family means to that individual child. Teachers who do not have intersubjective communications with children will not learn about their life experiences and will do a disservice by delaying or even damaging her/his cognitive development. Causing a child to become frustrated because of the failure to see the world from the child's viewpoint may eventually lead to a dislike for school. If teachers are unaware of intersubjectivity by definition, even if not by the term, it is important to bring awareness of its importance.

National attention, research, and policies strongly recommend bachelor's degree education for preschool teachers, birth to age 8 in all child care settings, both private and public 
(Institute of Medicine (IOM), \& National Research Council (NRC), 2015). In Illinois, the State Board of Education (ISBE), Illinois Board of Higher Education (IBHE), and the Illinois Community College Board (ICCB) announced the state's adoption of the IOM and NRC recommendation, and a strong push toward that end (Chadwick, 2015). The reality, however, is that Illinois, through its licensing standards, legally requires minimal education/training (Illinois DCFS) for all early childhood programs other than the state program (Preschool for All), run by the State Board of Education, and Head Start, mandated by the federal government to require bachelor's degrees for classroom teachers. There are three scenarios in which Illinois allows a person to be qualified as a teacher of children under the age of 6 . The two requiring the most education clearly illustrate a conflict between state entities/policy makers:

(1) sixty semester hours (or 90 quarter hours) of credits from an accredited college or university with sex semester or nine quarter hours in courses directly related to child care and/or child development from birth to age 6; or (2) one year (1560 clock hours) of child development experience in a nursery school, kindergarten, or licensed day care center and 30 semester hours (or 45 quarter hours) of credits from an accredited college or university with six semester or nine quarter hours in courses related directly to child care and/or child development, from birth to age six (Illinois Department of Children and Family Services Licensing Standards for Day Care Centers, Part 407, 2014).

There is also confusion about intersubjectivity in teacher education preparation textbooks, different terminology attributed to the concept, and different definitions tied to the concept. Therefore, this study explored implications for congruency in teacher training, employment requirements, and the materials used in education and professional development. 


\section{Purpose of the Study and Research Questions}

The purpose of this study was to explore preschool teachers' intentional, multi-modal communication methods to reach intersubjectivity with children before and during instruction in early childhood preschool environments, and to investigate the extent to which preschool teachers are aware of the continuous communicative process leading to intersubjectivity. Where awareness is expressed, how does it influence guided participation? The concept of intersubjectivity is minimally represented in many early childhood textbooks even though Vygotsky is considered one of the pillars upon which theory is translated to practice (Berger, 2018; Berk \& Meyers, 2021; Morrison, 2018). Vygotsky $(1978,1986)$ and many neoVygotskians who write of intersubjective interactions, using one term or another, indicate that instruction for concept development is considerably more effective when teachers/MKOs engage in guided participation while communicating to reach intersubjectivity (Brinck, 2008; Bruner, 1990; Luria, 1982; Sinha \& Rodriguez, 2008).

This study sought to shed light on interactions that are mindful of the need to reach intersubjectivity before and during instruction. Vygotsky (1978) indicated that intersubjectivity must be reached in order to effectively engage in concept development with a learner in the ZPD. If this is the case, engagement toward intersubjectivity should be noticed often during preschool teachers' day.

Over many years of observing preschool teachers in the classroom and reading of Vygotsky's (1978, 1986, 1997a, 1997b) work in texts, a salient behavior surfaced, as teachers endeavored to 'scaffold' learning in the ZPD, they tended to maintain teacher-directed instruction, and used close-ended questioning. The idea that children learn in the context of meaningful relationships (Shore, 1997) is misinterpreted by many preschool teachers as they 
continue to teach as they themselves feel is appropriate, usually in the way they themselves were taught. As a result, teachers 'instruct' without getting to know the children, their interests, and how they perceive concepts. The key preface to effectively developing children's knowledge intersubjectivity, is a communicative activity during which "two participants who begin a task with a different understanding arrive at a shared understanding. ... achieve true collaboration and to communicate effectively during joint activity" (Berk \& Winsler, 1995, p.27). Teachers who fail to consider the perspectives of children when engaging in joint (also known as mediated) activity cannot effectively enhance learning. This may be connected to a teacher's need to stay in control of her class. For example, I have witnessed teachers who, instead of discussing a conflict and working toward a resolution, tell one child to "say you're sorry." The child may comply without understanding why, especially if she comes from a home that has no problem with the behavior she displayed. Based on these experiences, there are three research questions explored in this study:

1. What are preschool teacher perceptions of guided participation/mediated activity?

2. How do preschool teachers intersubjectively communicate with children to assess their concept knowledge, and readiness to enter the ZPD?

a. How to teachers use knowledge of a child's culture to assess knowledge and readiness to learn?

3. How do preschool teachers intersubjectively engage in guided participation and mediated activity to further develop concepts in the ZPD?

a. How to teachers use knowledge of a child's culture to make connections to learning? 


\section{Research Design: Phenomenological Instrumental Case Study}

This study followed the interpretivist paradigm (Merriam, 2009), with the purpose of describing, understanding, and interpreting instructional communication and intersubjectivity in preschool classrooms. Glesne (2011) seems to have spoken directly to this study when she wrote, "The role of the social scientist then becomes that of assessing others' interpretations of some social phenomenon and of interpreting themselves, other's actions and intentions" (Glesne, 2011, p. 8). When it comes to preschool teachers engaging in intersubjective communication, they are acting as social scientists throughout the day. Documented observations of teacher-child interactions prior to and during instruction were coded to analyze intersubjective behaviors and discourse and interpret these behaviors and communication to inform early childhood educators about intersubjectivity. Understanding teacher-child engagement and the context of concept development is important as preschool teachers strive to facilitate children's development to their full potential.

According to Merriam (2009), qualitative, phenomenological studies strive to understand the whys and wherefores of phenomena, in this case, intersubjectivity. Intersubjectivity is phenomenological, in that it seeks common understanding between two or more people (Thompson, 2005) coming from individual perspectives of lived experiences (Husserl, 1970). This study sought to explore how teachers engaged preschoolers in joint referencing, active verbal and non-verbal discourse in order to arrive at mutual understanding, and to continue in that engagement as teachers partner with children in mediated activity, leading them to higher cognitive functions.

Moran (2000) indicated that phenomenology was formally presented by Husserl (19001901) "in discussing the need for a wide-ranging theory of knowledge...that had grown, by 1913, 
into an a priori transcendental science of pure consciousness as such" (p. 2). After 1927, the philosophical perspective that is phenomenology was largely considered to be "understood almost exclusively in terms of the combined contribution of both Husserl and Heidegger, and so it appears to Levinas, Sartre, Merleau-Ponte, and Derrida” (Moran, 2000, p. 2).

Choosing a phenomenological perspective for this study required me to stay open-minded even though I could not detach from my own background/history, cultural attitudes or explanations, and to carefully describe the phenomenon known as intersubjectivity (Groenewald, 2004; Glesne, 2011; Moran, 2000). I did my best to carefully, accurately, and richly describe, without bias, document, code, and apply hermeneutic procedures to communicative interactions between teacher and child in their search of mutual understanding; which is a day-to-day human experience (Groenewald, 2004; Moran, 2000; Wojnar \& Swanson, 2007). There are many types of phenomenology and, after reviewing possibilities, chose to follow along the lines of Husserl and Mead; Husserl taking the perspective that we live in a shared world - each other, things, environment, language, and meanings, and Mead, whose social behaviorist phenomenological methods compliment those of Vygotsky (Valsiner \& van der Veer, 1988).

Due to the scope of diverse pedagogical practices found in preschool teachers, this study considered three preschool teachers, their instructional events, and classroom environments as separate cases. Each case, defined in this study, was the preschool teacher's communication just before and during instruction (guided participation). Miles et al (2014) defined a case in many contexts including "a role" (p. 29), that is, the role of teacher.

Stake (1995) explained two elements of case studies: the case itself, and the issue(s). Studies that focus on the case tend to look at people or systems, for example. In this study, preschool teachers themselves were not being studied; their behavior in relation to 
intersubjectivity was studied. Therefore, this was an instrumental case study (Merriam, 2009;

Stake, 1995), constructed and conducted to encourage understanding of intersubjective communication between preschool teachers and children. The research questions were curiosities about teacher-child interactive communication that were investigated through case studies.

\section{Participants and Setting of the Study}

Time constraints translate to travel constraints, which categorized this study as one using convenience sampling. As such, the sample was selected based on distance, researcher financial resources for travel, availability of the teachers, and time needed to conduct the research (Merriam, 2009; Miles et al., 2014).

This study sought to observe and record teachers and children in their natural, preschool setting in order to capture interactions during guided participation (or what teachers determine is an 'instructional' period) as they occur on a day-to-day basis. According to Stake (1995), “Our first obligation is to understand this one case.... In instrumental case study, some cases would do a better job than others" (p. 4). Considering chances to maximize understanding, progressive programs, including Head Start, were considered more likely to employ teachers with higher degrees, therefore more training. In addition, self-proclaimed progressive programs in central Illinois (not necessarily Head Start) were likely to be Reggio Emilia inspired, aligned with The Project Approach, or follow the Montessori Method. Head Start is a nationally funded program serving low-income children with sites/centers to be found throughout Illinois. Reggio-Emilia inspired centers are listed on the North America Reggio Emilia Alliance (NAREA) and nearby centers are known in Illinois, Indiana, Kentucky, and Missouri. The Project Approach also inspires a number of early childhood programs throughout Illinois and neighboring states. Efforts were made to engage two of the three cases with any of these three progressive programs. 
The third case was from a traditional, high quality rated child care program, not aligned with an aforementioned approach. Rather, it identified its curriculum as emergent, aligning with play-based and child-centered learning. The third case held a prominent indicator of high quality in Illinois, defined by NAEYC accreditation and a gold circle of excellence through the ExceleRate quality rating system, which is Illinois' iteration of the national quality rating and improvement system (QRIS); a voluntary program assessment of quality ECE.

Classroom teachers were identified through email and telephone outreach. Efforts were made to observe and collect data on the teacher-child dynamic involving teachers or children from different cultural backgrounds in order to determine whether intersubjective communication is intentional. Theoretically, teachers and children with similar socio-economic or ethnic backgrounds will have similar knowledge references, or similar interpretations of concepts (Geertz, 1973). Therefore, teachers and children from different socio-economic or ethnic backgrounds are less likely to share mutual knowledge references without intentional, intersubjective, verbal as well as non-verbal communication to reach common understanding.

Participants were recruited and apprised of the ethics around qualitative research, and questions were ethically and respectfully answered (Merriam, 2009; Miles et al., 2014, Stake, 1995). Informed consent was completed before any action was taken. Participants were apprised about their control over the process; if they decided we are done, we are done. Interviewees were identified with pseudonyms on all recordings, notes, transcripts, and written results.

\section{Data Collection}

In this qualitative, phenomenological case study, the methods used to gather data were classroom observations, semi-structured interviews (pre- and post- observation with an optional second post-observation session), video of the classroom environments, and coded analysis of 
data. Observations of teachers engaged in instruction with children was as discreet as possible, in the natural comfort of their own classroom environment. Permission to video was sought as it added rich information to the data, notably through recording voice tones and inflections and non-verbal communications. Video recording was done from one placement and no movement around the room to minimize distracting the children and thereby negatively affecting the natural setting. Arrangements were made to focus on instructional interactions between teacher and child. I placed myself closer to teacher-child communications, taking notes and using a video recorder. Children were considered participants if they engaged with the teacher and then, only for the duration of the communication, verbal and non-verbal. Characteristics of voice offered evidence of understanding that was captured in the video recordings that included tone, volume, inflection, and speed of speech. I did not seek additional discourse with the children in keeping with observation-only methodology. Great effort was expended to observe teacher-child communication early in the semester anticipating that, while teachers may know some children very well, more may have entered into the classroom for the first time, lending itself to a different level of communication. However, observations and interviews were done midsemester, and expectations were that teachers should continue their efforts to engage in guided participation, as learning is continuous, and does not diminish as the year progresses. Evidence of intersubjectivity in the classroom environment was documented through video recording to investigate learning materials, toys, books, projects, artwork, and a variety of materials to illustrate each child's and family's inclusion in the classroom/program community.

It was anticipated that intersubjectivity would be evident throughout a preschool day, as children, in classrooms where teachers employ developmentally appropriate practices, are continually investigating, exploring, observing, and theorizing (Edwards et al., 2012; Helm \& 
Katz, 2001). Teacher-child interactions were observed during preschool mornings when the teacher's plans of learning opportunities are typically implemented. Teachers were visited in the spring semester; two times for semi-structured interviews, and the classrooms were visited two times for observing teacher-child instruction, and again to video the environment when children were not present.

In order to embark on a journey to explore how preschool teachers, knowingly or unknowingly, engage children toward intersubjectivity, I needed to investigate teacher-child communication in the classroom, and also interview the teacher to get their perspective about communication in the big sense (including non-verbal methods, and attention to emotions) and what understanding looks like. Teachers participated in 45-50 minute semi-structured interviews to consider their communicative experiences with children in their classrooms, their philosophy of teaching, to clarify questions from the observations, and to learn of cultural differences between teacher and children.

Interview questions were open-ended unless I needed specific, clarifying information, such as a particular child's SES or cultural background to further interpret a communicative episode. The interviews themselves were conducted in a location of the interviewee's choosing, with encouragement that it be isolated to avoid any chance of being overheard, or to put the person at risk. I was not an active participant in the classroom activities, although regrettably, my presence will have an impact on the dynamics of the classroom. I experienced more questions and requests from children (not those interacting with the teacher) on second-day observations that required me to redirect them with the promise to explain later (I did).

Data collection included videotape of observations and the environment, and anecdotal notes during audio-taped interviews and videotaped instruction. Teachers were invited to review 
and critique data summaries. After transcriptions were completed, recordings were deleted, and transcripts were coded, analyzed, and locked in a desk in my home office.

\section{Data Analysis}

Two coding applications were utilized in the first coding cycle. Emotion coding revealed evidence that Vygotsky $(1978,1986)$ and others (Bodrova \& Leong, 2007; Zlatev, 2012) indicate to be a major factor in the development of higher order functions. Process coding was useful in that it "extracts participant action/interaction and consequences" (Miles et al., 2014, p.75), perfect for a preschool classroom full of learners. Gestures, facial expressions, intonation, body movements, and other non-verbal communications were noted carefully, as they contributed to the entire message being expressed by preschool-aged children (Vygotsky, 1978). Coding of interactions during instruction interpreted methods and purpose of communications (including attitudes), and coding of interviews interpreted the participant's descriptions, purposes, and attitudes about communicating with the children. Whether teachers recognized their efforts to reach mutual understanding with children to develop their conceptual knowledge, or why they engaged the children; this study sought to uncover intersubjective communication in its many iterations.

First cycle codes were written descriptions, analyzed for patterns, such as a teacher's shortened verbal interaction when children hit, or extra smiles and hugs when a child puts extra effort in solving a problem. The patterns were accompanied with narrative descriptions to add context to the interaction. Time, place, activity, and even other people in proximity likely influenced the interactions. Context is needed to understand the intersubjective nature of communication. Notes from observations and interviews were transferred to large hand-written coding sheets, along with quotes from participants. Throughout the analysis phase, notes were 
added to coded data as results unfolded. Video of classroom environments were also descriptively coded on large handwritten sheets, offering a triangulation of the phenomenon through interviews, observations, and classroom that speaks to confirmation of the findings (Miles et al., 2014). Interview questions were assigned to research questions. Coding sheets were arranged by research questions with collective descriptions from interviews, observations, and environment. All three teachers chose not to have optional second interviews and all three declined review of data summaries.

Triangulation of data in qualitative research serves to develop accurate interpretations. According to Stake (1995), 'We need certain triangulation protocols or procedures which researchers and readers alike come to expect, efforts that go beyond simple repetition of data gathering to deliberative effort to find the validity of data observed (p. 109)." Evidence of actual teacher-child verbal and non-verbal communications may or may not provide evidence of a teacher's intentionality toward intersubjectivity. The teacher may be aware of the concept 'serve and return' from the Harvard Center on the Developing Child (Serve \& Return, 2017), and has yet to fully understand its relation to intersubjectivity. She may not be cognizant of her own attitudes toward the children, or how to expand the serve and return concept in the classroom via culturally responsive learning materials, learning activities (individual or through guided participation), or in everyday casual conversations. If, for example, a teacher consistently engages in verbal interaction without gestures, this may indicate misunderstanding of intersubjective communication. If there is also evidence that classroom art made by a diverse group indicates children making the same item in the same way (perhaps something from Pinterest, a popular go-to 'resource'), this further lends validation that the teacher does not comprehend intersubjective concepts. In contrast, teachers whose children are represented in 
class materials (books, art, learning items), who are respected as the individuals they are, and who are instructed with references the child knows (e.g., apartment vs. house; park or street vs. yard) are more likely to reflect knowledge of intersubjectivity. This study made efforts to meet the expectation of Geertz (1973), “A good interpretation of anything - a poem, a person, a history, a ritual, an institution, a society - takes us into the heart of that of which it is the interpretation (p. 18).”

\section{Ethical Issues: The Role of the Researcher}

It is imperative that researchers clearly define their roles which are, according to Glesne (2011) "situationally determined, depending on your philosophical perspective, the context, the identities of the participants, and your own personality and values (p. 59)." Roles specific to case research are identified by Stake (1995), who first states that 'all evaluation studies are case studies (p. 95).” The phenomena of intersubjective communications between preschool teachers and children are the case studies in this research and were evaluated as to their effectiveness in establishing mutual understanding between interlocutors.

To a limited degree, this study will demonstrate myself as biographer. During the course of semi-structured interviews, we learned about each preschool teacher's educational background to include knowledge and training about intersubjectivity, and the processes involved. We learned about the teachers' attitudes, values, and practices toward cultural differences, albeit subtle, and they are all key elements in the development of effective interactive communication.

Interpreting the dynamics of intersubjectivity is of particular importance to me because I feel the phenomenon has not been fully explained to the early childhood community. From Vygotsky's theory to neo-Vygotskian research, intersubjectivity has found a niche in linguistic disciplines, leaving early childhood professionals and practitioners missing key elemental 
knowledge about assessing readiness for the ZPD, and how to effectively guide learning within the ZPD. Moreover, I feel the importance to mutual understand what intersubjectivity means, and how 'scaffolding' in its general definition, lacks the concepts and complexities regarding intersubjective communication.

As one of most contemporary qualitative researchers, I hold a constructivist view of knowledge-building (Stake, 1995). Interpretation of data in this case study depends on the construction of knowledge starting with Vygotsky's socio-historical (aka socio-cultural) theory of cognitive development, specifically, concept development in the ZPD. Where the concept of intersubjectivity developed in the disciplines of psychology and linguistics, it was lost in education, seemly replaced with the misunderstood term, 'scaffolding.' Based on my 30+ years of experience in early childhood professions, I find it difficult for practitioners to change or expand a definition once it is established. This is partly due to the plethora of literature available in old interpretations of concepts. Therefore, every effort was made to minimize using the word 'scaffold' in this study. Linguists define 'intersubjectivity' with uncomplicated words; 'mutual understanding' (Trevarthen, 2008), which Vygotsky $(1978,1986)$ enveloped without separate definition, within the concept of 'mediated activity' taking place just outside and within the ZPD. Concepts and activities critical to constructing knowledge of intersubjectivity begin with Vygotsky's theory, then use Vygotskian and more contemporary guidelines to evaluate and interpret the phenomenon. In addition, data from the case studies were used to develop descriptive guidelines for intersubjective communication to be used in early childhood education, including higher education teacher education programs.

Qualitative studies tell the story surrounding data. For that reason, every researcher will bring their unique culture to the research - their own principle of relativity (Stake, 1995). In like 
manner, each reader will bring his/her unique culture to the interpretations of research. Striving to approach the data of this study from different perspectives is intended to deepen the readers' scope to understanding the concepts presented. The intention is that we come to mutually understand intersubjectivity, and developmentally appropriate practices to reach that goal.

\section{Summary}

Cultural historical theory and its researched components discussed herein brought attention to state educational systems, such as the Illinois State Board of Education, and national professional networks, such as the National Association for the Education of Young Children (NAEYC). Contemporary education practices require teachers' pedagogy to be evidence-based (i.e. research to practice), and standards-based, both professional (e.g. NAEYC, IPTS) and curricular (e.g. IELDS, WIDA). Within professional and curricular standards, we find fragments of Vygotskian theoretical and research knowledge, such as collaborative learning, pretend play, and guided participation, incorrectly labeled as 'scaffolding.' Teacher Education programs tout Vygotsky as one of the giants in the field of education; he is the person who brought attention to culture as a factor of learning, he joins Bruner and Piaget in constructivist ideas, he describes learning as a dynamic that occurs in the zone of proximal development (ZPD), and that learning is a collaborative process. What gets very little space on a page is the depth of his conviction that language, indeed the larger concept of communication, is critical to learning. Many studies, books and articles on intersubjectivity are found in child psychology, developmental psychology, linguistic development, and cognitive development databases. It's time to bring this work, and terminology, to the realm of education, where Vygotsky $(1978,1986)$ intended, as per his writings on pedagogy during the last few years of his life. 
Initially, Vygotsky $(1978,1986)$ paid little attention to non-verbal language as a critical factor in the development of understanding. His successors (Karpov, 2005; Rodriguez \& Moro, 2008; Rogoff, 1990, 2003; Trevarthen, 2008), expanded his ideas and discovered levels of intersubjectivity beginning at birth which describe the deep, emotional aspects of social interaction and that relationship to instructional learning.

Not only does language play a major role in learning; the importance of language is to engage children in the development of concepts, which takes years to evolve. True (adult) understanding of concepts, says Vygotsky $(1978,1986)$, begins in adolescence, when children are capable to share concepts via verbal sharing of abstract representations. It takes a broad understanding of concepts to be able to articulate abstract representations. A child needs to put many experiences, emotions, vocabulary building, collaborations, and guided participation in the ZPD under her belt to get to mature understanding.

On the way to mature understanding, there are steps toward, or levels of concept development, beginning at birth, that are necessary, as cognitive development is a continual journey, not a stage. Intersubjectivity, being a natural effort to arrive at mutual understanding with another interlocutor, is key to developing higher order thinking. Imagine a child who does not develop a full understanding of sorting, or number sense; it's happening in our schools today, and it is connected to emotional self-regulation - the emotional aspect of which Vygotsky writes (Berger, 2018; McClelland et al., 2007; Vygotsky, 1986, 1978).

Exploring intersubjectivity was an effort to uncover some interesting information about teaching and learning. This study may have implications for professional development of early childhood educators; it may call attention to the need to expand textbook information. At the very least, it will call attention to the need for further study. 
Intersubjectivity should be intentional. Every early childhood teacher should have a clear understanding of the need to engage children in rich, clarifying, emotional, enjoyable communication that references the child's own background, ergo, points of reference. The purpose of this study was to seek teachers' intersubjective behaviors and their understanding of the process. Without evidence of intersubjectivity being practiced in our preschool classrooms, a child's development of concepts is at risk. Teachers may be shortchanging children's cognitive potential and development of higher order thinking. 


\section{CHAPTER IV: FINDINGS}

Findings of this study are discussed in relation to the theoretical framework, which is Vygotsky's process of communicating toward intersubjectivity in order to facilitate a child's concept development. In this framework, first the teacher and child reach intersubjectivity of the child's prior knowledge. Then teacher will take the child and their shared understanding of prior knowledge, into the zone of proximal development (ZPD) to scaffold concept development (Vygotsky, 1978; Zlatev et al., 2008). It is essential that intersubjectivity be maintained throughout teacher-child interactions for instruction to be effective (Vygotsky, 1986). I will also connect findings to research questions one, two, and three. This study sought to investigate intersubjectivity and consider its implications for professional development. Three preschool teachers were interviewed and observed to explore these questions:

1. What are preschool teacher perceptions of guided participation/mediated activity?

2. How do preschool teachers intersubjectively communicate with children to assess their concept knowledge, and readiness to enter the ZPD?

a. How do teachers use knowledge of a child's culture to assess knowledge and readiness to learn?

3. How do preschool teachers intersubjectively engage in guided participation and mediated activity to further develop concepts in the ZPD?

a. How do teachers use knowledge of a child's culture to make connections to learning?

To study intersubjectivity, three preschool teachers were recruited to participate in two semi-structured interviews and two classroom observations. The first interview was intended to learn about each teachers' training and pedagogical style, determine their knowledge base of 
terms such as guided participation, their perspective on communicating with children (especially during instruction), and how culture factors into understanding.

Between the interviews, I was permitted two visits to videotape the teachers with children during their regularly scheduled center time for a minimum of 30 minutes. The purpose of the videotaping was to collect evidence of intersubjective communication and scaffolding. The second semi-structured interview was intended to follow up on the videos (intersubjectivity and scaffolding), and to seek clarity of the interactions, such as how the teacher understood the child's perspective during instruction.

Using Merriam (2009) and Stake (1995) as guides in developing research questions, I decided to employ both generalized and focused questions to maximize the collection of evidence regarding teachers' knowledge and use of guided participation, or mediated activity while engaging young children's learning.

\section{Teacher Demographics}

Denise is a Head Start preschool teacher who teaches four- to five-year-olds and holds a BA degree in Early Childhood Education. She was a volunteer over 20 years ago and stayed involved with this program as a parent, and then teacher. KA is also a Head Start preschool teacher of four- to five-year-olds and holds a BA degree in Early Childhood Education. She has been teaching at Head Start for over fifteen years. Denise and KA are employed at separate Head Start programs in different counties. Ann teaches preschool in an institutional child care program. She holds an AAS in Early Childhood Education. Ann works in a mixed-aged classroom, and right now, the enrollment is two- and three-year-olds. She has worked in this program part-time for over ten years and secured a full-time position at this same center two years ago. 


\section{Teacher Perceptions of Guided Participation/Mediated Activity}

For the initial semi-structured interviews, participants were asked to describe their teaching styles, whether they were teacher- or child-directed. Anticipating that the terms 'guided participation' and 'mediated activity' are not familiar with many early childhood educators, I began with more generalized and related questions about teaching style. The rationale was to see how, in their responses, participants made connections between teacher directedness and guided participation. In their follow-up interviews after two observations, participants confirmed their teaching style and were asked what led them in that direction.

Next, participants were asked directly about their feelings and knowledge about guided participation (i.e., mediated activity), and if they include this teaching strategy in their classroom. In the event I misinterpreted their responses about teaching style, stating the concepts after discussing teaching styles may have provoked knowledge of the terms. In a related question, participants were asked to describe their role in children's cognitive development. I ended this section of questions asking about communication. First, I asked to learn ways in which teachers communicate with children, especially during instruction. Then, why they communicate in these ways and how cultural factors are considered in these methods.

\section{Denise}

In both her pre- and post-observation interviews, Denise described her teaching style by referring to the program's Creative Curriculum and their program standards manual of school readiness goals. She said she develops weekly themes and lesson plans and that those plans reflect goals that connect to the school readiness manual, such as patterns. Asked to describe how children are engaged in learning, Denise shared an example of how she introduced their current theme, living and non-living things. 
We talk about the theme and brainstorm what they like and dislike about the unit. The children had to tell us things that are living and things that are not living. Then, we went around the room and had them point to things that are living and things that are not living.

Next, I directly asked about Denise's knowledge and feelings about guided participation, also known as mediated activity. She connected the terms to "hands-on learning," and continued, "When they're guided is like if there is an instructional format. What we do is instructional format; we give an example and say, 'this is what we're going to do next."' Rounding out the questions about guided participation, I asked about ways Denise communicated with children, especially during instruction, and followed it by 'why.' She was very concise and did not elaborate, stating "open-ended questions, song, finger plays, and books." To the question of why, Denise said, "children need hands-on and visual learning cues to help them learn."

In our post-observation interview, Denise was asked about her motivation to engage in teacher-directed instruction both days. She related to the school-readiness goals of literacy and math (patterns and K-W-L). Denise used non-verbal communication when she pointed to the empty outlined boxes on the work paper as she asked, "What comes next." Asking how the children received her verbal and non-verbal communications during instruction, Denise referred to the first child doing patterning. "I felt she understood and then she was having some problems. That's why I extended the activity to say, 'ok, now that you have patterned this, what do you think will come next?"' Denise explained that is why she continued to ask, 'what comes next' to all the children during that task. "I wanted to make sure everybody was getting it."

The final two questions to Denise were about understanding; first, how she determined whether a child understands her, and then, how did she understand a child's perspective and level 
of knowing during instruction. In the video, there is a point at which a child (not the one she was working with) brought over his work. Denise engaged in active listening and confirmed that she "was checking in" to see if he understood. Therefore, active listening is a way Denise determines understanding of both teacher and child. Then, as she shared more about the video, Denise focused on connecting 'level of knowledge' to 'following instructions.' For example, she stated, "Their level of knowledge is, they could identify the animals that I placed in front of them, and that's by using pictures and real-life pictures and stuff like that."

To summarize, Denise gave very brief responses to questions even when encouraged to give examples. After few attempts, I stopped asking for clarification to avoid the risk of her separating from the study. Denise's responses indicated that she was not informed about guided participation or mediated activity. She did state, "guided...if there is an instructional format." She is correct that it is an instructional format. However, she followed with "this is what we're going to do next," inferring that her concept of 'guided' is 'directed.' It is not clear whether she was aware how communication is used to determine prior understanding to accompany a child into the ZPD to deepen concept knowledge. She referred to the Head Start standards manual to substantiate her use of direct instruction. Denise used direct instruction during observations except when completing the K-W-L chart for their farm theme.

\section{KA}

During KA's first interview, she indicated her utilization of both teacher- and childdirected instruction as well as co-teaching. KA described co-teaching along the lines of Vygotsky’s (1978, p. 86) concept of some children functioning as "more capable peers," also referred to "more knowledgeable others," to their classmates. She indicated, "I'm there but they are helping each other." KA was asked to describe how she engaged children in learning. She 
talked about using visuals such as using puppets. She went on, "we use a bell that rings and... it means it's time to clean up. We have rules and we try to give warnings [before there are transitions]." KA spoke about using cooperation activities in Sunshine Circle, "and we actually feed the children. There is a process, a certain way things are done and they're done that way for a certain reason." Another form of engagement KA used was singing. She stated that she, "always sings transitions because music connects [children to motion]." The songs that KA shared were all directive, telling children what to do, whether it was setting the table, cleaning up, or preparing for outside time.

KA indicated that, once the children are acclimated to the classroom and their daily routines, she was more likely to ask open-ended questions. "This is a hypothetical. Somebody asked about the farm, the goats, and why they were eating grass." She replied, What do you think we could do to find out about the goat, you know, so it's more of a planning time? I try to make it more planning time for them. Or an idea, a sharing kind of thing and we are always in small groups, so I'm teaching two or three at a time. I have a specific goal or specific objective I'm working on because when we do our outcomes it seems like literacy and math were where our numbers [child assessment scores] were the lowest. In a lot of situations where they're doing literacy and math, we're combining them. How many letters are in your name? Whose name is longer? Whose name is shorter? You know, do you see any of the same letters in someone else's name? There's a lot of how many so we try to incorporate both into that as well.

KA's response to the direct inquiry about knowledge and feelings about guided participation aka mediated activity was to question, 
I guess guided would be...the environment is... set up? You set the environment up and...see where it's headed, so the children kind of guide it?" She continued, "The other way, the more mediated to me would be more, 'we need to learn to count...ten blocks. You're still doing it in a fun way, but you've got a goal. You're not letting the children guide it.

In her post-observation semi-structured interview, it was noted that KA's teaching style while engaging a small group of children in Zoo Phonics was virtually all direct instruction. For example, she put a Zoo Phonics animal on the floor, pointed to it, and asked who that was and what sound started his/her name. She built short words and pointed to each animal for the children to repeat the letter sound it represented. Her explanation was that, There is a good mix of children who can visually do something but there's also two or three in the group that have to have the tactile; they have to be able to move things. I was working with a group of children who were at all different levels.

KA added that she wanted, "peer teaching going on," so she "gave them the opportunity of trying to flood the lesson with as many different things as I can so I can reach all the children.” KA did not clarify 'peer teaching' other than referring to herself being the person who had to guess the zoo animal's first letter sound and motion.

To determine whether there was a connection between communication and understanding, KA was asked how she determined whether a child understood her during instruction. Instead of explaining how, she said she "can keep a mental note." She went on to add,

Normally if I was collecting data, I want to know what this child knows, I would have either [written] it down immediately, or I would have done it in the classroom within 
earshot of someone else who could record so I could put it in the computer and then have the portfolio. I'm fortunate enough, small enough group [five children], my brain works that way. My brain is just mapped like that. CHILD One knew all the letters of the alphabet, she understood the concept of at - /a/t/ is at, /i/t/ is it, and /o/t/ was ot.

Next, I asked how KA understands the child's perspective and level of knowledge during the experience. She replied, "Well, I think they are engaged and that was the important part." The engagement KA referred to was verbal responses and whether the children performed the motions paired with the animal first-letter sounds. KA acknowledged that on Zoo Phonics day the children were getting restless. "The two younger ones in the group were having a difficult time. That's when I switched it up to movement. The transition became part of the lesson." Movement was recorded as making the large and small motor motions for letter sounds. KA used facial expressions as she directed the children to respond. For example, she made a 'wondrous' face when asking what letter sound an animal's name started with. Her eyebrows were raised, her eyes widened, and her voice pitched higher.

The act of facilitation could involve co-teaching and open-ended questions, as in facilitating (to help bring about) problem solving or learning. KA did exhibit knowledge of the facilitating learning. However, it did not lead to evidence of guided participation. During an observation, KA sat at a table where manipulatives were in an adjacent shelf. Two other children were at the table using paper and writing tools. KA had a plastic basket from the shelf and asked the children, "Look at my basket. What happened?" One of the children said, "I did it. I don't know...I didn't know which ones..." KA responded, "You didn't know which ones? Would you like to help?" These were a set of twenty-six acorns. On the caps were upper-case letters, on the bottom were lower-case letters, and then there were twenty-six items, the names of which started 
with one of the letters. These were to be placed inside the acorn. In response to KA's question, the child moved over next to KA to help put the acorns back together. KA said, "We're going to have to put our heads together." KA offered a statement and an open-ended question. "We have to put all these together, so how are we going to do that? What can we do first?" The child took an acorn, to which KA acknowledge, "Can we do that? Oh, what's in there?" There was an item in the acorn that did not belong there. The acorn was returned to the basket. KA asked, "How can we organize this... what would be the easiest way to put it together? What do you think we could do, because there are an awful lot in there?" The child took another acorn from the basket and turned it over to see nothing inside. She returned it to the basket. KA said, "Maybe, oh, that would be a way to work it out. Put the bottom of the acorns over here [placing a piece on the table], and the tops of them over here [placing one in another place on the table], and then we'll look at the little things inside and..." The child took an item from an acorn. KA said, "What is in there?" The child held it in her hand. "That's a $/ \mathrm{j} / \mathrm{jar}$. Then, pointing to the acorn from which the child retrieved the jar said, 'Oh, that's not a lower case ' $\mathrm{j}$.' From here KA continues to direct the child in the task of placing the pieces in their rightful place.

What started as facilitation turned quickly to direct instruction. KA and the child worked for twenty-three minutes and by then the child demonstrated little energy or interest in completing the task. Her head was bowed, and she responded to KA's questions with what appeared to be guesses. KA asked the child if she was done, and the child nodded in the affirmative. KA said she could go play, and she did. In the follow-up interview, KA stated she knew the child who offered to help was the child who took the acorns apart. KA wanted her to be responsible to put them back together. 
In summary, KA's responses at the first interview indicated potential knowledge of guided participation and communication conducive to establishing prior understanding before taking a child into the ZPD to scaffold learning. She used the terms 'co-teaching,' 'facilitating,' and 'open-ended questions.' KA described co-teaching as, "I'm there but they [the children] are helping each other," and there was an example of an open-ended question whereby she asked how she and a child could find out about goats. No co-teaching was observed, and, while openended questions could have meant facilitating instruction with the child working with plastic acorns, KA did not engage in conversation that would empower the child's lead in her own cognitive development.

Ann

Ann indicated that her teaching style was in concert with emergent curriculum; that she creates her curriculum around, "more of what they [the children] want to talk about and that they want to learn about." In her view,

the amount of knowledge that the children get from doing it that way is so much better than if you are like, OK, it's apples and leaves and fall and winter and snow. We talked about bugs with two- and three-year-olds for four full months! And yes, there were the worms that ate the apples, but we weren't only talking about the apples. Its incorporating what's going on outside and maybe what you, as a teacher, want to teach. You are teaching through what the children are wanting to know about and the knowledge they want to get, and to give.

Ann's approach to engagement was making sure there are materials throughout the room that are of interest to the children. Using examples from the children's interest in bugs, Ann indicated she was "going to add more bug books, add bugs to the sensory table, and add them to 
the science center." She explained that while the children will sing bug songs, she expanded their vocabulary by asking questions like, “How many legs does this ant - this insect - have?" Ann added, 'It's easier to engage them [children] if you're teaching them something they're interested in as opposed to trying to make them learn. They are directing me. I ask, 'what can I get you, or what do you need?'”

Based on her interview responses and two observations, Ann had a better understanding of guided participation (i.e., mediated activity). In the initial interview she indicated,

Guided participation is more when you're guiding the child in the direction that you want them to go. Mediated is more of where you are there but you're not making them - you are letting [Ann's emphasis] them make their decisions and their choices. Mediated is, you're standing back and you're more of a facilitator, allowing them to go where they need to go instead of where you need to go, or where you want them to go.

Observing Ann, I saw that she engaged the children in teacher-directed as well as childdirected guided participation (further details of the interactions will be discussed under research questions two and three). Video recorded evidence related to how she determined whether the children understood her during instruction. I saw that Ann was attentive to the children when they spoke. She looked at them. She listened. Ann employed active listening, open-ended questions, acknowledgments, observations, gestures, and non-verbal communication. Employed in developmentally appropriate ways, and in reference to Vygotsky's writing on the concept of intersubjectivity (Vygotsky, 1978, 1986) these qualities raise the probability that Ann and the children understand each other.

I was interested in how Ann understood the child's perspective and level of knowledge during their experiences. As we reviewed the videos in the post-observation interview, Ann 
pointed out examples showing the children employing much the same strategies she did while communicating. I noticed that there was less active listening on the children's part, although there was evidence of a child adding Ann's words after saying, "yeah.” Through observations, I noted the children's gestures, facial expressions, and that they demonstrated what they were doing and thinking. Engaging conversations using non-verbal and verbal communication was recorded throughout the two days of observation in Ann's classroom.

\section{Summary}

In addition to asking whether the participants are familiar with the term guided participation or mediated activity, and to get clarity about their understanding of the terms, Denise, KA, and Ann were asked to describe their teaching styles and to describe ways they engage children in learning. If the terms were unfamiliar, evidence of their engagement strategies would help determine whether they are practicing guided participation. Using examples to enhance their descriptions was encouraged so participants and myself could reach intersubjectivity. Asking questions about verbal and non-verbal communication was crucial to determine effective guided participation.

Based on the evidence gathered in two interviews and two observations, it is possible that Denise and KA have had some exposure to the concept of guided participation. Denise mentioned hands-on learning, which is often connected with child-centered learning. However, she did not expand her response, and it is possible that hands-on meant gluing pictures of animals in pattern squares. Denise directed the children through their instruction without actively participating in any observed experiences. KA used the terms child-centered and co-teaching, both of which can be attributed to guided participation. KA did participate during instruction on both observation days. On the first day, she told the children how to test her own letter/letter 
sound knowledge. On day two, KA held the basket of acorn pieces close, dominating the search for pieces. She decided whether an item, an acorn cap, or an acorn bottom would be introduced to the child in their search for matches. In these ways, the participation was still very teacher directed.

Furthermore, when reflecting on KA's observations, I noticed that the child eventually reached over to pick up pieces and investigate the item or letter. The child working with acorn pieces participated, it seems, because she felt guilty that she left the pieces apart the previous day. KA said they had to be put back together and that is when the child offered to do the task. The initial open-ended questions were quickly answered by KA, who verbalized her thoughts about how they should proceed. Further, an opportunity for her to allow children to co-teach (based on her own definition in the initial interview) was not realized. KA's communication included facial expressions although it was demonstrated throughout direct instruction rather than in true conversational engagement. For example, KA used 'oh,' and 'oooooo' often and these verbalizations were accompanied by pursed lips, downturned head, and wide eyes. Gestures were limited to large and small motor Zoo Phonics animal letters on the first observation day, and to upturned hands on the second.

Denise and KA had similar responses to these first queries. Ann identified her teaching style as aligned to emergent curriculum and her responses were congruent with guided participation. Emergent curriculum is the American term equated closely to guided participation that uses guided construction of knowledge that requires a two-way relationship between teacher and child (Biermeier, 2015). According to Ann's interview responses, she engages children in things in which they are interested. She uses open-ended questions to learn what the children already know and have experienced, then builds on that knowledge through exploration and 
investigation with her guidance. Ann indicated that she engages children using open-ended questions, gestures, introducing new words, and reminders. Ann shared that reminders are her way of getting a child's focus or attention by "rubbing them lightly on the back, a little tap on their foot, talking with them and laughing with them." Two observations provided evidence that Ann engaged children through guided participation. The term she used was, 'emergent curriculum.' The idea of guiding in the term 'guided participation' is that the teacher will make observations, acknowledgments, use gestures while communicating, wonder about things, and ask open-ended questions to the children can make the discoveries, and do the investigating. In that sense, the children are guided to their own cognitive development, being in control of their learning. In brief, Denise's teaching style was non-participatory, teacher-directed instruction of program-determined content. KA's teaching style was participatory, teacher-directed instruction of program-determined content. Ann's teaching style was participatory, child-directed instruction of child-centered content.

\section{Intersubjective Communication to Assess Readiness and Engage Learning in the ZPD}

Research questions two and three, which also question how participants use cultural knowledge of children in the process, are more effectively discussed together since one follows the other. As a reminder, research question two addresses how preschool teachers intersubjectively communicate with children to assess their concept knowledge, and readiness to enter the ZPD. Research question three probes deeper to explore how preschool teachers intersubjectively engage in guided participation/mediated activity to further develop concepts in the ZPD. In this section, I will reference participant interviews and observations as they align with research questions two and three. 
First, the interview questions that sought to connect teacher strategies and pedagogy to these research questions included, a) what information teachers wanted to know about their students in order to be an effective teacher, and how they used that information; and b) different elements about learning objectives, a child's readiness for the objectives, and whether/when a child demonstrated a learning objective. The communicative strategies and their relation to cultural knowledge was discussed in question one and is also considered in this section. While teaching styles and knowledge of guided participation may raise the likelihood of early childhood educators communicating to intersubjectivity, they are not requirements. In other words, educators whose dominant style is direct instruction can still engage in intersubjective dialogue during instruction. However, reaching common understanding is not the end of this query.

Vygotsky's theory of cognitive development indicates that intersubjectivity is required for teachers to assess a child's readiness to enter the ZPD (Vygotsky, 1978). In addition, it is critical for effective teachers to reference a child's cultural background when applicable to reach intersubjectivity. Without this level of communication there is no guarantee a child understands the teacher or the concept/objective of instruction. Once in the ZPD, it is teachers' charge to maintain intersubjectivity and increase the depth and/or breadth of the child's conceptual knowledge that is being investigated.

Moreover, teachers who engage children to reach common understanding will be positioned to determine whether the child is ready to enter the ZPD, where the teacher will scaffold learning in a particular behavior/skill. For example, if a child and teacher speak the same first language and the child is known to see and correctly identify a blue ball in a storybook, an effective teacher may realize that identifying the color of one item is not an 
indication that the child understands 'blue' in the more general sense. With knowledge of cognitive development, the teacher realizes that children can pick out a certain colored item from among other-color items before that child is able to correctly name the color of the item in isolation. Further, there are many shades of blue that make it necessary to introduce the concept in different contexts; different shades of blue and different items that are blue. Therefore, if teacher, through conversation over time and using items that are blue, discovers the child has no knowledge of blue other than in the storybook, the child is already in the ZPD. In determining the child's prior concept knowledge, teacher began introducing the child to other contexts in which there exists the concept 'blue.' This teacher can use the blue ball in the story to isolate the concept of color. That is their intersubjective base from which to scaffold knowledge of 'blue.'

To further illustrate this point, consider a teacher who, referencing a page in a picture book, asks the same child, 'what things are blue' and the child answers incorrectly. An inappropriate reaction to this would be if the teacher concluded that the child does not know the color. The teacher did not check to see if the child could pick out blue from among other 'same' items (all crayons, all counting bears) before making a conclusion. This teacher did not seek intersubjectivity. Therefore, according to Vygotsky $(1978,1986)$, the teacher will not be effective taking the child into the ZPD to scaffold (expand the depth and breadth of) the concept of 'blue,' as the child may be confused as to what the teacher is trying to convey.

In the following sections, I will summarize data gathered from Denise, KA, and Ann related to research questions two and three. 


\section{Denise}

Denise indicated that knowledge of a child's background is important to meet each child's needs. There was no expansion on the statement. I asked how she chose to introduce these urban children to a unit about the farm. Denise said,

In our curriculum it talks about the children being able to identify things that they see daily of a different environment, so what we did is they know things from the city so we were going to teach them about the farm, that's part of their community; such as where does their food come from, where the plants come from, where the different animals live. That's why we chose the farm.

Denise indicated that the class already spent previous weeks on nutrition and where food comes from. She went on,

We already talked about where food comes from, so one of the kids said, 'where do the animals live?' When the children give a question, you want to scaffold their learning; you want to investigate and explore where the animals came from.

In a sense, the children put themselves in the ZPD by inquiring about where farm animals live. Being city-dwellers, none of the children [according to Denise] experienced seeing animals on a farm. Denise's class was scheduled to visit a farm to conclude their unit.

Prior to the first observation, I asked Denise to identify ways in which she communicates with the children, especially during instruction. "Open-ended questions, songs, finger-plays and books" was her response. When asked why, she responded, "Children need hands-on and visual learning cues to help them learn." In contrast to these interview responses, asking questions and active listening were her sole means of instruction on the first observation day and, one by one, 
they glued paper animal illustrations on a four-square ABAB pattern outline. "What's this one (animal illustration) and what comes next" were the most used questions of engagement.

To explore understanding of pedagogical concepts, I asked Denise about her understanding of guided participation or mediated activity. Her interpretation was that they are "...hands on learning. If there is instructional format, we give an example and say, 'this is what we're going to do next." I asked, "when does this happen?" Denise replied, "It happens at circle time, and it happens in small group, and it also happens when they're in free time or play time."

The instruction observed in the next two days was expressly about farm animals. After large group circle time, the children were welcomed to play in centers. Denise set up her instructional opportunity and called one child over at a time. The first day she took green construction paper, cut in half horizontally, with four squares traced across the page (Figure 3). Denise had "Baby Animal Pattern" printed on the top of the paper. To the side were glue bottles. Clipped to each prepared paper were two each of two different baby animal cut-outs; baby horse, baby sheep, baby cow, and baby goat. In other words, children had two pre-selected animal pictures to work with.

Figure 3

Baby Animal Pattern

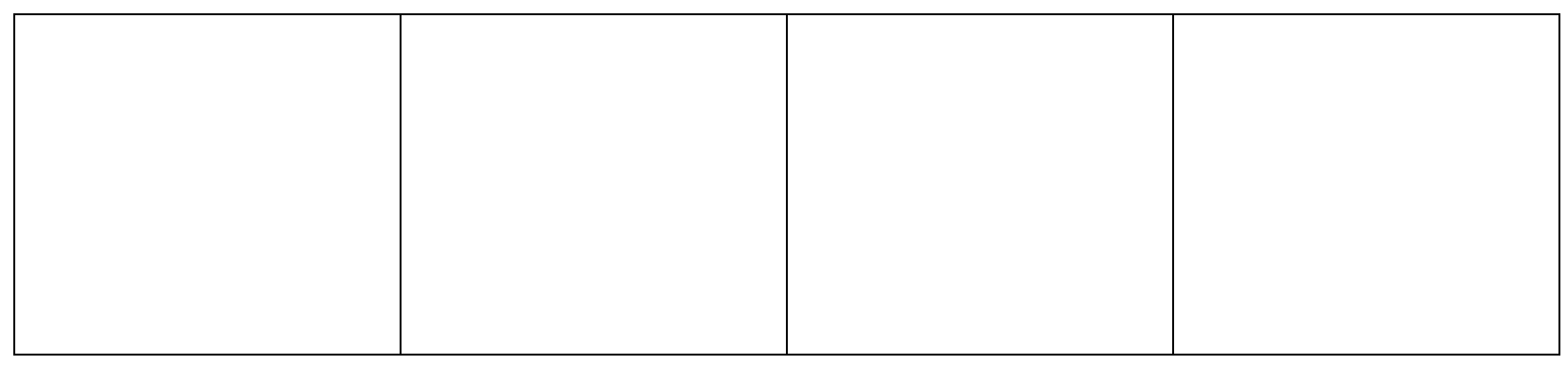

One at a time, children were called to the table. She began by reminding the children they are working on farm animals today. To one child, Denise showed him and said, "Today, you 
have a...." [showing the child an animal]. He answered, 'horse.' Denise engaged in active listening and repeated the child's response. She showed him the other animal. She said, "This is a..." and the child answered, 'sheep.' Denise repeated the child's response. At this point, Denise determined that the child correctly identified pictures of the select animals. She then shared, “Today we are going to pattern. Look, 'Sherai' made this one. [pointing as she said] She patterned cow, goat, cow, goat.” The interaction continued:

DENISE: You're going to pattern. Which animal first?

CHILD: [pointed to horse]

DENISE: What is that?

CHILD: Horse

DENISE: Okay, you're going to put the horse here [pointing to the first square on the left and handing the child a glue bottle]. Put your horse there [pointing again].

CHILD: [followed direction]

DENISE: Then you're going to put your [moved the picture of a sheep closer to the child]...

CHILD: Sheep

DENISE: Where are you going to put that one?

CHILD: [pointed to the second square]

DENISE: Good job.

CHILD: [put glue on paper, picked up the sheep picture and placed it on the glue in the proper square]

DENISE: [pointing to the pictures as she spoke] You have a horse, sheep.... What goes next? 
CHILD: [picked up the sheep picture]

DENISE: Look, look. If you pattern your horse, and sheep, what goes next?

CHILD: [touched sheep]

DENISE: No...look [points at the third square]

CHILD: [touches the other picture not yet placed]

DENISE: What is that?

CHILD: Horse.

DENISE: The horse will go where?

CHILD: [touches the third square]

DENISE: Good job.

CHILD: [glued the horse in the third square]

DENISE: Good job. What goes next?

CHILD: [pointed to the last picture not yet glued]

DENISE: What is that?

CHILD: Sheep

DENISE: Good job. The sheep goes next.

CHILD: [glued the sheep in the last square]

DENISE: Tell me about your pattern. [pointing as she speaks]. Denise says the animal and waits for the child to repeat] Horse...[child repeats], sheep....[child repeats], horse...[child repeats], and [pointing to the sheep she waits another moment...

CHILD: Sheep.

DENISE: If it went on, what would go next?

CHILD: Uh? I don't know. 
DENISE: Okay, try again. [pointing] We did horse... [child did not respond]... sheep... horse...sheep....What would come next?

CHILD: ehhhhhhhh

DENISE: Sheep or horse?

CHILD: [points]

DENISE: What is that?

CHILD: Horse.

DENISE: That's right. Good job. High five. Good job. Go back and play.

Was there intersubjectivity in this conversation? There was, as far as identifying or remembering pictures of farm animals. These illustrations were cartoon-like, as those you would see in a young child's coloring book. However, the objective of this instruction was patterning. Regarding an understanding of pattern, there were missed opportunities to communicate the concept and check for intersubjectivity. The child did not indicate an understanding of patterns.

According to Vygotsky's writing (1978) the teacher should step back if the child gets frustrated. This child did not show any frustration, nor did he indicate knowledge of patterns. This does not mean the child was prematurely placed in the ZPD. He may well be able to understand patterns. Using intersubjective communication, the child may have grasped - or remembered - the concept of patterns in the ZPD and would practice the cognitive skill over time, deepening his understanding in varied contexts and complexity.

The second day in Denise's classroom I observed her developing a K-W-L (know-want to know - learned) chart. As before, Denise called the children to the table one by one to write on the chart. Asking the three questions, Denise wrote the children's responses on the chart. In this activity, Denise gave no preface to the K-W-L of farm animals. The questions were open- 
ended and related to farm animals, which was the topic for days. A few children did not respond after the question. Denise did not give the children cues to remember what the child might know. Some did not have a response to the 'learned' portion of the chart. The term was not explained. If they had no answers, Denise offered them ideas, to which they agreed. She wrote those ideas on the chart and dismissed the children to "go back and play."

The K-W-L chart was a complete verbal experience. There was no conversation or nonverbal communication. There were no props to use for understanding of context or to spark a point of interest. Perhaps the children's concept knowledge was considered a given because it was the topic in class for a few days. However, the concept that challenged the children may have been verbalizing their own interests. In two days, the children are mostly doing what they are told or have a choice between two or three things.

During the first observation, Denise engaged children through direct instruction in group time and in the morning activity. Questions were closed ended, as she sought one 'correct' answer. The activity was patterning. All but two children made an ABAB pattern with farm animal cut-outs (living). At our post-observation interview, Denise confirmed that this was the children's second or third experience with patterning. Denise offered, "one of the children was more advanced. She did two [AABB patterns.]" The pattern grid Denise prepared had four boxes, limiting the instruction, although she asked some children 'what comes next' indicating an extension off the boundary of the construction paper.

My second visit to Denise's classroom provided further evidence of direct instruction. Children were brought to the table one by one after large group time, as in the previous visit. Denise had a modified K-W-L chart prepared and asked the children three questions based on that day's group time. The theme was still living and non-living things. Today, the class began 
an investigation of 'The Farm.' She asked, "What do you know about the farm, what do you want to know about the farm, and what did you learn today about the farm?" Every child gave one response, and a few got help to expand their statement. For example, a child answered, "a cow" to 'what do you know about the farm?' Denise asked, 'Do cows live on the farm?' The child nodded and the sentence Denise wrote was, 'cows live on the farm.' When children answered the three questions they were told to go back where they were playing, and the next child was called over. Both observations showed clear evidence that Denise engaged children in direct instruction. Since conversation - serve and return - was absent, there was no evidence of intersubjectivity.

We talked about the children's exposure to patterns at the follow-up interview. Denise indicated that the children were shown patterns "once or twice" over time and it was all instructional. Vygotsky $(1978,1986)$ writes that learning happens in the ZPD when meaning is attached. The children may have wanted to learn about farm animals. However, where is the meaning of placing pictures of farm animals in $\mathrm{AB}$ patterns? Further, did the children get a chance to understand pattern with one repetition? There was no point of reference to offer the children in their task to pattern farm animals. Asking 'what comes next' is not enough. Communicating to intersubjectivity in the form of using props, referencing children's background knowledge about animals in general, and investigating patterns while playing could have brought the children to a more productive level in the ZPD. Referencing the K-W-L chart, I asked why it was done at that point in the unit. Denise indicated it was part of the curriculum and she would go over it when the class gets done with the theme. They planned the farm visit for the following week. 
Also in our post-observation interview, Denise was asked to share why she engaged children using direct instruction, the style she agreed was employed during instruction. She referred to the Head Start standards manual indicating teachers look there to review readiness goals. "The children need to learn how to pattern [a readiness goal] so that's what we worked on." Continuing the topic of direct instruction,

AES: "What led you in this direction?"

DENISE: "We would go into the program manual of standards and look at school readiness goals. In the goals it talks about being able to pattern and so the children need to learn how to pattern. So that's what we worked on."

AES: "Is this (referring to the video of my observation) one of their earliest experiences with patterning?"

DENISE: "Yes, that was probably their third experience."

AES: "OK. It is still ABAB."

DENISE: "Yes, then one of the children did two (extended animal illustrations in a continuing ABAB pattern). She is a little more advanced. That's why she did two." Denise acknowledged that this was a teacher-directed activity. I mentioned that there did not seem to be opportunities for conversation. She replied, "They were supposed to pattern and then move on (to another activity). When you came back the next day (for the second observation) that was where we talked about what we know and what we learned."

Joint attention is a useful strategy in guided participation/mediated activity. I pointed out that Denise engaged in joint attention to draw the children's attention to the animal illustrations. I continued, "You were next to the child, using your hands and your voice." Referring to the video, "we can see the children watching what you are doing. It's joint attention." Denise 
replied, "yes." Without intersubjective communication, however, it is unclear whether the children and Denise were truly on the same page of understanding regarding $\mathrm{ABAB}$ patterns or the meaning of a K-W-L chart.

$K A$

When asked how children's background information is helpful, KA discussed home visits and how many children in her class are new to a preschool classroom. She chose to highlight two specific children's anxiety early in the school year. KA mentioned a child who benefitted from having a photograph of his parents in his cubby, "When he needs his mommy." Another child had early fearful experiences that causes him to be fearful when there are loud noises or at "chaotic" transition times. KA kept an eye on this child and offered support when she saw the child "jump out of the chair." There was no other mention of the value of children's cultural backgrounds while the children were in preschool.

KA described herself as a teacher who uses teacher-directed and child-directed instruction. I first observed KA's instruction during Zoo Phonics. She began by showing the children small plush animals, asking what they were, and reviewing the first letter sounds and gestures as per Zoo Phonics. [Later KA explained that, instead of using letters from the merchandise package, she used small plush animals that resemble beany babies.] After a few reviews, she placed an animal on the floor and asked the children, "Who is this?" The children responded in unison, "Allie Alligator," and KA made the Zoo Phonics gesture and the sound "ă." Next Timothy Tiger was put to the right of Allie (at). She spoke and gestured at the same time, "t," repeating it three times. The children repeated with her. KA then reminded the children that two letters make a word and asked. "What did we make?" The children followed KA as she made each letter sound and said “/ă/t/, at! This is at." Within five minutes the children became 
restless as they sat on the floor. One child went to the basket to look at an animal, KA sang "everybody sit on the floor." After this exercise, Bubba Bear was placed to the left of Allie and Timothy, making, “/b/a/t/. Bat!” The children got through 'bat' and 'sat.' Then KA changed Allie to Inny Inchworm and directed the children through 'sit' and then 'hit.' By the time Inny was placed the children were squirming quite a bit. KA asked them to sit and look at her or at the animals on the floor. The children were not given an animal or any learning materials (such as laminated letters to match the sounds). The animals were in a basket at KA's side.

At the ten-minute mark KA changed the instruction. She took two minutes to verbally explain what the children were going to do, then KA put five animals in the middle of the circle. She closed her eyes and a designated child chose an animal from the five (one for each child) and told KA to open her eyes. KA was to name the animal, e.g., Timothy Tiger, and say the first sound along with the body movement. KA guessed the wrong animal or offered the wrong gesture with every child, encouraging them to help her get the correct answers. The children's attention started wandering. KA redirected them to sit or look at her. Zoo Phonics instruction continued for twenty-five minutes.

There was complete teacher direction during instruction of Zoo Phonics. Considering the children's shortened attention, self-regulation challenges, and responses (or lack of responses), the concept of meaning surfaces. Shore (1997) wrote, "Children learn in the context of meaningful relationships" (p. 33). These children were reciting letter sounds without seeing letters as reference. Small plush animals were laid side by side and the children were instructed to make words based on their position. There was one instance of intersubjective potential. One of the children's names began with 'A.' When Allie Alligator was brought out the child said, 
"that's my name!" KA responded, "Yes, it is." That was the end of the connection. KA was focused on the drill of saying letter sounds, and of making them into words.

The second observation with KA involved her working with one child, then another at the manipulatives table during center time. KA had a small basket of plastic acorns in front of her. The acorns are made in two parts with a capital letter on the cap of the acorn, and the lower-case letter on the bottom. A third piece, an item whose name begins with one of twenty-six letters, is to be put inside the acorn. There are seventy-eight pieces to this letter and sound recognition material. A child sat at the table drawing when KA approached.

KA: What happened to it?

CHILD: I did it. I don't know how... which one goes together.

KA: You didn't know which one?

CHILD: Yeah.

KA: Come sit over here. We're going to put our heads together.

CHILD: [complies]

KA: So, we have to put all of these together. How can we do that? What can we do first? CHILD: Picks up an intact acorn and shakes it [no sound].

KA: Oooo [using facial wonder with pursed lips and wide eyes], is there something in there?

CHILD: [no verbal response] opens it, holds it to KA to see inside. Child looks at KA. KA: [gestures with two upturned hands and a surprised expression] Nothing. [looking at the basket] How can we organize it to figure out.... What would be the easiest way to put it together?

CHILD: [looks at two disconnected pieces] 
KA: What do you think we could do?

CHILD: [shifts the position of the basket]

KA: There's an awful lot in there.

CHILD: [takes another connected acorn from the basket]

KA: Is there something in there?

CHILD: [opens the acorn and shows KA]

KA: [upturned hands and surprised expression]

CHILD: [holds the empty parts up to KA]

KA: We have some together; we just don't have anything in there. Maybe... oooooh, I have an idea. What if we put the bottom of the acorn here [making a place on the table] and the tops of the acorn here [in another place]. And then, we could look at the little things inside and....

CHILD: [shows KA an opened acorn]

KA: [surprised] Ooooooh, what's in there?

CHILD: Cup

KA: A $/ \mathrm{j} / \mathrm{j} /$ jar. So what letter is that?

CHILD: I don't know. [hands KA the bottom of an acorn]

KA: Oh, [hand over her mouth] Wait a minute...[smiling]...that's not a lower case ' $\mathrm{j}$.'

This is a representation of the exchanges between the child and KA. The child was given approximately one to two seconds to respond before KA proceeded with the 'lesson.' The child made efforts to put the pieces together with KA's direction. There were many close-ended questions, and the child demonstrated different levels of engagement throughout the fifteenminute activity. Early on, two children came over and asked if they could help too. KA 
redirected them back to their previous center. This was a lost co-teaching opportunity.

Reviewing the video carefully, the child was, at first, quiet and waited for KA to answer her own questions. At the seven-minute point, the child became more verbal. She identified two letters on her own. She took an item from the basket in front of KA. It was a star. The child said, "It's a star! It means the letter 'S!' Now the child looked for the two acorn parts for 's.' She looked in acorns and was distracted by another letter. KA said, 'Let's look for that 'S,' let's not get sidetracked. Do you know what that means? Do you know what getting sidetracked means?" The child held another acorn bottom up to KA, who looked inside and said, "Who put a $/ \mathrm{n} / \mathrm{n} /$ nest in here. This is all messed up. Keep looking so we can find that lower case 'S."' With clues from KA to "check on the table," the child located the acorn parts and put the set together. Next, KA asked the child to count the completed sets (four), and how many were orange (2), green (1), and blue (2).

KA consistently offered items to put into an acorn that were not a letter match. These non-examples were paired with playful smiles by KA and yet the child looked at her silently. At fourteen minutes into the activity, the two other children returned, stood by the table, and joined in on sorting and searching for matches. KA said, "Thank you for your help. I think we've got a plan." One of the other children said, "I can help," and handed KA a piece the original child was told to locate. KA responded, "I know you can, but I want her to find it." The item was put into the group of items. KA said, “OK friend. One more. Let's see if you can find one more letter and I'll see if somebody else wants to come play with me." KA helped the child complete the set for 'A,' and she looked at the child and said, "Let's count how many we did." The child counted, one to one correspondence, to seven. KA said, "Awesome. Six plus one is.... seven. [The child 
had not answered.] Do you want to keep going or are you all done?" The child put fingers in her mouth and said, "done." She left promptly.

A child who offered help earlier returned, and KA asked if she would like to work on the acorns. The child affirmed, seeming eager to search letters and match items. KA continued to ask close-ended questions, although this child was able to explore pieces independently, perhaps because they were all sorted on the table. KA's teaching strategy was otherwise consistent with the second child.

As mentioned, engagement for both children was not limited to matching letters and then matching first sounds of items to put with their corresponding acorn. Once an acorn was complete the children were asked to count them. When another set was completed, they were asked to count again, after which KA stated, for example, "Six. Five plus one equals six.” KA also drilled the children on colors of the acorn caps. "How many are blue," she asked, continuing until all the represented colors were counted, and totaled once again. When center time was over, nine acorn sets were completed.

On both observation days, KA clearly engaged children using direct instruction. Other than the child who verbally recognized her 'name,' and was fearful with the grasshopper and insect items, there was no personal connection between the children's lives and their cognitive experiences. They were being drilled to remember letters, letter sounds, and counting to perform tasks of word-making and addition. It is in keeping with a statement KA made at our first interview, during the question about teaching style. She indicated,

I've got a specific goal or a specific objective I'm working on because when we do our outcomes [child assessments], it seems like literacy and math are where our numbers were the lowest. Every day they're getting math and literacy and then in a lot of 
situations, we're combining literacy and math. How many letters in your name? Whose name is longer? Whose name is shorter?

Considering KA's intersubjective engagement of children over two days of observation, there was no evidence of relational discourse during instruction that would assist children in finding meaning of the concepts she presented. The children engaged in Zoo Phonics did not have the opportunity to see print letters during instruction. Letters and sounds were verbalized, accompanied with the small and large motor Zoo Phonics gestures for each letter-sound. The plush toys were not the same as those found in the phonics materials, and that may have caused some dissonance in understanding. KA did not offer relationships between letters and children's names, for example, even when a child demonstrated that connection on her own. That child found meaning in a letter. The children who helped get the acorn letter/item sets together may have missed opportunities to make meaning in their task. It could be the quantity of pieces was overwhelming and needed to be pared down. Using the Illinois Early Learning and Development Standards (IELDS, 2013) as a guide, and knowing that the children did demonstrate knowledge of at least a few upper- and lower-case letters, perhaps a different approach to this experience may have yielded a different outcome. Below is the relevant learning standard:

Demonstrate an emerging knowledge and understanding of the alphabet.

4.B.ECb Recognize and name some upper/lowercase letters of the alphabet, especially those in own name.

4.B.ECc With teacher assistance, match some upper/lowercase letters of the alphabet.

Ann

Discussing how background information contributes to her effectiveness as a teacher, Ann mentioned that knowing about a child's home environment explains much of their 
disposition at school. If she knows the dynamics at home, it helps her understand how to address a child's behaviors if needed. For example, a child started "acting out" and it was out of character. Ann shared,

They have an aunt living there and it's hard for her, seeing the feelings of her aunt and cousins. It's also good to know their [all families'] cultural background so you include the family and learn more about how their family life goes; things that are important to them, not just the things you know about, or things that are important to you.

Ann aligned her teaching style to emergent curriculum, child-centered experiences presented by teachers who engage children through guided participation. It is the intention of the teacher who engages in guided participation to actively communicate with the children to gauge prior knowledge and reach intersubjectivity before taking children into the ZPD where deeper understanding of concepts percolate and generalize. To be successful, teachers and children must understand each other. Teachers learn from children, and children learn from teachers (Biermeier, 2015; Gandini, 2012).

At our post-observation interview, it was noted that Ann engaged the children in both direct instruction and guided participation. Prior to my first observation Ann explained that among the children's interests that day were the roots of the baby tree on the table. She stated that in previous days the children investigated seeds by sorting and observing their attributes. They used their own and new knowledge to consider plants' need for sun, water, and dirt. Each child was given a choice of one kind of seed to plant. That child remembered that you do not see the roots of plants and he was curious. "He leads a lot of their play and ideas. [He is their moreknowledgeable other, MKO.] If they see that he is interested in it, they become interested in it." 
She presented an apple and the baby apple tree that was planted by a previous class the semester earlier. Ann's direct instruction began with an apple and apple cutter. As Ann spoke about another class looking at apple seeds and planting one, she cut open the apple. "Where are the seeds?" she asked. The children saw none in the pieces Ann gave them to eat. The older child pointed to the core and looked at Ann. She said, "It is called the core," snapped it open and gave a piece to each of the four children who shared two magnifiers.

Returning to the baby tree (it was approximately twelve inches long, end to end), Ann said that one seed planted in dirt grew into this baby tree. She showed them the roots, stem, and leaves. One child stayed with the baby tree and observed it with a magnifier. Another child asked if he could get the play dough, to which Ann said, "Of course. Let's play with it on this end of the table so (child's name) can look at the tree." Within a few minutes all the children were playing with play dough. Ann facilitated this part of the day engaging in guided participation to reinforce the dynamic of a classroom community. For example, "How can we make sure all our friends have play dough?" This prompted one child to give everyone a piece he cut from a bigger piece. Ann supported him to count how many, so everyone got a share.

At different times, children brought rocks and shells to the table and used them to explore with the dough. Ann engaged every child, observing what they were doing with the dough (e.g., "I see you are squeezing that dough") and adding gestures (squeezing). She responded to many comments using active listening. The children watched how their peers approached the play dough. One copied another, and one commented on another's work. Impressions of the shells were made. Rocks were covered with accompanying stories and one early-three-year-old child engaged with Ann for a few minutes about the dough:

CHILD1: I squeeze it. 
ANN: What does it feel like when you squeeze it?

CHILD1: It feels better.

ANN: It makes you feel better when you squeeze that?

CHILD1: Uh Huh. At my house. I squeeze play dough at my house.

CHILD2: I squeeze play dough at my house too.

ANN: You squeeze play dough at your house?

CHILD1: Yeah

ANN: That makes you feel better when you squeeze the play dough?

CHILD1: Yeah.

ANN: So, do you squeeze it when you're happy?

CHILD1: Yes.

ANN: What about when you're angry?

CHILD1: And really really mad.

ANN: When you're really, really mad?

CHILD1: Then my mom and my dad.

ANN: So, you squeeze your play dough?

CHILD1: Uh huh and I get really angry in my mouth. Then I get big mouth.

ANN: Uh Huh

CHILD1: .... and a big tongue.

ANN: Uh Huh [in follow-up, Ann shared she was unsure what the child was telling her at this point]

CHILD: ....and big teeth.

ANN: Yeah? You get a big tongue and big teeth. And so, you squeeze your play dough? 
CHILD: Yeah, and then it just choke me.

ANN: Oh...when you get a big tongue and big teeth?

CHILD: Yeah and (child demonstrates coughing with tongue out)

ANN: When did that happen? When do you get a big tongue and big teeth? When does that happen?

CHILD: I don't know. Playing with it and... (the child makes a mouth gesture as if eating something, mouth open and closes with a nasal-throaty sound) and make my choke and (makes a coughing sound with tongue out again and put his hand to his throat). ANN: Oh, you put the play dough in your mouth, and you choked on it at home? Is that what you're saying?

CHILD: Yeah.

ANN: Oh, let's not put the play dough in our mouth.

CHILD: Yeah. He made a face with pursed mouth and scrunched nose.

ANN: Let's only put food in our mouth.

CHILD: Yeah.... We need...we need... and he got distracted by another child squeezing her dough and focused back on his own.

During his communication with Ann, the child's story was heard. Ann actively listened to this child, keeping her attention toward him. He had something important to share with her and she stayed with him until she realized he shared an experience he had at home. Ann and the child reached mutual understanding in this conversation. Ann notified me about her conversation with this child's parent, who reported he had put play dough in his mouth, which caused the child to gag and cough, presumably because of the high salt content in the dough. Both Ann and the child know each other a little more deeply now. 
Observation two took me to Ann's class the next day. One child from observation one was not there, and another child was there this day and not at the first observation. I arrived as morning meeting was ending. Ann said she had something to show the children and brought over a large tub with small pots inside. Next to the tub is a very large pot almost full of soil, and a hand trowel. Throughout the experience, CHILD1 is attentive yet remains quiet, watching and listening.

ANN: Look at the seeds!

\section{CHILD2: Big!}

ANN: They are. Look at how big they are. (To one) Come over here so you can see.

CHILD2: Sprouting.

ANN: They are sprouting. I like that word you used. You used the word 'sprouting.' If these are sprouting up, what's happening in the dirt too? There's a picture over there (referring to a photo poster behind them of a plant and the parts above and below the soil). [the children turned to reference the poster.] What's gonna happen underneath, do you think (hand to chin)?

CHILD2: There're roots.

ANN: There are roots. Do you think they will have enough room in here (gesturing to the small seed-starter cups)? The top part will grow taller and taller (gesturing by raising her hand a little higher and a little higher).

CHILD2: Uh Huh

ANN: You do? I think that's a good idea that you think the roots might be OK but, for the plant to grow up, we need to make a bigger space for them to grow down. See the 
picture there (the children turn to reference the poster)? The plant grows up and the plant grows down. So, we have to make sure it has enough room to grow down.

CHILD2: Smiling, OK.

ANN: But what should we do?

CHILD2: Points at the soil in the bucket.

ANN: Do we need to put them in there?

CHILD2: nods in the affirmative.

ANN: Let's see how many plants we have that sprouted. [As the children are counting with Ann, she observes a sprout emerging from the planted seed. Ann points.] This is a sunflower seed, and they grow very high.

CHILD2: [sees that two others also have seeds still attached and reaches to pull one off.] ANN: We need to let that grow some more.

CHILD2: [child withdraws her hand]

ANN: Look at these leaves. Are they the same as these?

CHILD3: No, these two are the same (points to two examples of the same plant).

ANN: That's right, $\underline{\text { CHILD3 }}$, they are the same.

CHILD2: They are the same.

ANN: We need to let these sprout and get a little bit bigger.

CHILD2: These ones are the same.

ANN: Look at that, they are the same.

CHILD3: [gesturing] These ones are not growing.

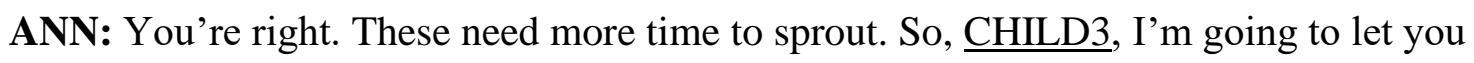
make the first hole in here (gesturing to the large pot) to plant this flower (specifies one). 
CHILD3: [used his finger to poke a hole in the soil]

ANN: Look at that hole. Is it big enough for this flower?

CHILD3: Yup, like the seeds.

CHILD2: No

ANN: Do you mean like we planted the seeds CHILD3?

CHILD3: [nods in the affirmative]

ANN: Let's see, I'm squeezing the sides of the cup to take the flower out and...

CHILD2: It's bigger [the plant and surrounding soil]!

ANN: [takes the baby flower and surrounding soil out, puts it by the hole made by

Child3] Is the hole big enough CHILD3?

CHILD3: No

ANN: What should you do?

CHILD3: Make it bigger.

ANN: How could you make it bigger?

CHILD3: [pokes his finger deeper into the soil]

ANN: I don't know if your finger can make it big enough (the trowel is right by the child's hand in the soil).

CHILD4: [points to the trowel]

CHILD2: [picks up the trowel]

ANN: Can you help, CHILD2?

CHILD2: [puts the trowel into the soil and digs...]

ANN: There you go, the shovel made the hole bigger.

CHILD2: [with a turn of the wrist, soil flies out onto the floor] 
ANN: That's OK. We can clean that up. That's why I put plastic on the floor.

[The hole is completed]

ANN: OK, I'm going to put this in the hole, and you all have to pack the dirt in around it.

Do you know what that means?

CHILD2: Mmm-hmmm

ANN: Alright, now push that dirt in around our little flower here. Push the dirt...some from over here [guiding the children, three of four are participating]. Ann puts her hand on a child's saying, push it in. There we go, we don't want to cover it up.

CHILD2 was asked to dig another hole and she approached the pot energetically. Ann asked her to "be careful, dig the other hole away from the flower" they just planted. The first flower was covered up and Ann reminded CHILD2 to "dig gently." Another child uncovered the first flower and CHILD2 finished digging. Ann placed another plant into the hole and said, "OK, pack it in gently." Ann watched silently as the children packed the soil around the plant. Next it was CHILD4's turn to dig a hole. Ann guided her to an open place in the pot and, with one comment to be careful about surrounding flowers, CHILD4 completed her hole. Ann placed the third plant and said, "OK, pack it in just like before."

Video indicated that Ann observed one more child had yet to make a hole for a plant. CHILD1, the youngest, whom Ann later said does not like to get dirty, sat with Ann. Ann held CHILD1's hand with the trowel. CHILD3 got up quickly and said, "I'll get the garden gloves." Ann said, "Oh that would be a great idea CHILD3. Ann had the plant in the pot by the time CHILD3 came back to the experience. The children counted the plants in the big pot, and Ann observed that the soil is very dry. CHILD3 said they need water. Ann acknowledged CHILD3's memory and pointed to four cups with water in them on a shelf. CHILD3 was asked to get a cup 
and water the flower he planted. Each of the other children retrieved a cup with water and fed their respective flowers, even CHILD1.

Concluding this experience, Ann asked the children where they could place the pot so it can get sunlight and water on a rainy day. Ann reworded the question and CHILD2 said, "By the tree!” Ann said, “if we put this by a tree, will it get a lot of sunlight?” CHILD2 said 'yeah,' and Ann said they can try that tomorrow when they go outside. For now, Ann put the pot by the door with magnifying glasses so the children can observe their experience and think about the flowers that will grow. They were reminded that the flowers must stay in the dirt so they can grow. Ann said, "Please don't touch them. Look with your eyes or with a magnifying glass."

The children were engaged planting baby flowers for over 14 minutes. They were guided to wash their hands while Ann picked up the plastic and moved the materials where the children can observe. Two children got magnifiers and went to observe the newly planted flowers. CHILD3 declared, "I think they're growing!" Ann replied, "they are growing already? My goodness." CHILD3 said, "It grew!" Ann went over and said, "it did! Wow." The children gravitated to an activity table and took out play dough. Everyone appeared to enjoy working with play dough for the remaining 15 minutes I was there, until the children prepared to receive lunch. Ann remained responsive and respectful, engaged in guided participation.

The children in Ann's class were engaged in learning experiences with peers on both observation days. She used open-ended and close-ended questions, and made observations, wonderments, and acknowledgments in reference to the concept(s) of interest. Ann looked at the children when she spoke to them, using expression and tone. She waited for responses and listened, actively and also with added comments. 
The children were not only focused on the instruction presented by Ann, but they also learned about each other and each other's families in some respects. Looking at the baby apple tree, children acknowledged whether they liked apples and had them at home. One child was intrigued by the roots of the tree, and Ann made sure to mention and gesture how they work "like a straw" to bring water to the top parts. Investigating the interaction of play dough with rocks and shells, Ann commented about how a child completely covered a rock with the dough. Another child did the same, inviting the first child to "look at mine." Ann commented, "you both covered a rock. They are the same."

Observation day two expanded the children's experience with plants when they each had a chance to plant a seedling they previously started, into a much larger pot. They did not see the roots this time. Ann reminded them of the tree the day before and directed the children to a photo poster close by where they could see the parts of flowering plants. The same child who was interested in the apple tree roots pointed indicating they are there, inside the dirt. Ann acknowledged by active listening, adding, "they are holding the plant so it doesn't blow away." After the planting, the children recognized the need to get them outside in the sun to grow.

In the post-observation interview, Ann mentioned how the children adopted different preferences on nurturing the plants. "CHILD1 sings to the flowers, influenced by a video where a child sang to a seed, wanting it to grow. The more she sang the bigger it got. Then there's CHILD2, who is more scientific, interested in the parts." The children are constructing knowledge in their own way. Ann was not telling them what to do, except to please refrain from pulling the plants from the soil or eating play dough. Even the play dough, where the children gravitate after their experience time, offers Ann opportunities to intersubjectively engage learning. Ann explained that "play dough is completely their choice. I just follow them." Here, 
the children unwind and interact. They talk about their pets and help each other through the fear of snakes. One child has a family pet snake. Ann continued, “....and it's in the family pictures, and wears a cap and she pushes it around in a stroller." Through conversation, the children were able to reach mutual understand as to how a snake could wear a hat and ride in a stroller. There is a photo in the classroom by the family bulletin board. The fearful child and Ann talked about their cats. Another child reported about his dog. The intersubjectivity here is that families may have animals living with them. They are pets, and they are not all dogs or cats.

\section{Summary}

Considering the process of guided participation, it begins with the need for teachers to establish a child's level of prior knowledge through verbal and non-verbal communication. Even though a teacher knows a child well, it is reasonable to recommend that she 'check in' with the child on that day at that time, to see if they are interested in engaging in the instruction. Having a positive relationship between teacher and child affects verbal, non-verbal, and expressive discourse. Teachers' disposition should be genuine and show interest in the concepts being introduced or practiced. When the teacher determines the child's readiness to enter the ZPD, the intersubjective discourse continues which may include modeling, demonstration, encouragement, acknowledgment, open-ended questions - guided participation - where the child's knowledge of the concept of study grows. Contexts must be varied so the child develops meaning and therefore generalization of the concept. In this way, the child applies the concept in other contexts

independently (Vygotsky, 1986). When children demonstrate knowledge through their behaviors independently and without guessing, it is generally held that they understand.

The children in Denise's class may be able to name illustrated animals. The evidence cannot determine that they understood $\mathrm{ABAB}$ patterns. They were not given time to ponder the 
questions before Denise intervened, offering two choices. There was no conversation about patterns, only that they were making one. The children were all successful in making their pattern, $\mathrm{ABAB}$, because they had two pairs of two animals to choose. If they guessed the wrong answer when Denise said, "What comes next," there was one left to choose. Denise concluded the children's patterns with, "Good job." Rather than a learning experience, the KWL chart was completed as a task. The children again were called over one by one, responded, or were given a response to each of the three questions and were sent back to play.

KA was as directive, and more talkative, than Denise. The children were either right or wrong. KA worked with a group of seven to eight with Zoo Phonics in which the children were asked questions about the names of letters, the sounds of letters, and to decipher three-letter words, all with small plush animals and no symbols. There is no clear evidence whether the children can identify letters. There were seven children in the group, which made it difficult for KA to assess the children's success in assigning the correct sound to each letter because they did everything in unison.

The individual child tasked to help KA put the acorn sets back together did identify a few letters and a few letter sounds. The child had difficulty identifying many items that represented the letter, making it hard to complete sets. KA was consistent in verbalizing the first sound in the name of the item. There was no conversation about the item to give the child a clue of its name. As mentioned, this child may have been in the ZPD albeit without the benefit of making meaning. This instructional session is seen as similar to Denise's patterning. A few children identified animals for Denise, and the one child identified some letters and one item for KA. Both teachers used the drill to skill strategy rather than scaffolding concepts. 
Denise worked with children one on one to get tasks done. These tasks were linked to developmental standards, and the children's work was evidence of accomplishment. KA worked with a group and then with one child on literacy with a touch of math the second day. Both Denise and KA connected the emphasis on these two domains of learning to their assessment outcomes and school readiness goals. Even though I observed play in both classrooms on both days, there is an implication that these two teachers are more comfortable directing children's learning, which is quite a contrast to Ann.

Ann has what she calls 'instructional time' every day. It is part of the daily routine, as it is with Denise and KA, who work with children after large group. Ann did her instruction after snack which included conversation about what the children were currently investigating. I was able to witness this both days as I entered the room to set up the video. As part of the table conversation, Ann brought up things the children already did, such as deciding whether they wanted to grow a vegetable or flower, sorting seeds, and planting seeds. The children made comments that related to their choice of flower or vegetable etc., and Ann intersubjectively engaged.

Ann may not have realized that she was preparing the children for the ZPD, where they would inspect a live baby apple tree's parts and needs or plant their seedlings in a large community planter to continue the nurturing process that included singing! In this community of learners, the children owned their learning. Ann facilitated through guided participation that included intersubjective discourse with each child. The children in Ann's class demonstrated meaning through their communication and behavior. They participated because they were invited, supported if needed, and encouraged rather than directed. Observing Ann produced evidence that she used knowledge of a child's culture to intersubjectively communicate with 
children to assess their readiness to enter the ZPD and, while there, to further develop their concept knowledge.

\section{Conclusions}

The purpose of this study was to investigate intersubjectivity in the context of intentional preschool teacher and child communication, in early childhood environments. Interview questions presented to three preschool teachers sought evidence as to whether they knew the term 'intersubjectivity,' and 'guided participation,' or had a working knowledge of the concepts as a pedagogical approach to instruction. The study further sought evidence as to whether teachers practiced guided participation during instruction, even if they were unaware of the terminology. Based on their interview responses, Denise and KA may have had a vague understanding of guided participation, although they did not demonstrate that knowledge in the classroom. Both said that a child's background is important, although they valued it for reasons other than guided participation. Denise said it is used, "to develop and find out where to meet the children's needs." KA connected background knowledge to build the home-school connection. Ann echoed Denise and KA, and added that she uses background information to engage families in the learning process, to relate learning to a child's background - being aware of "where they're coming from," and sometimes "if they need an extra hug or extra understanding because they're going through things they don't understand themselves."

Intersubjectivity, readiness for the ZPD, and scaffolding concept knowledge was demonstrated by Ann, not only because she used the child-centered approach to learning. She engaged the children in conversation, as Denise and KA might have done even though they were directing the learning. Denise and KA could have assessed readiness through intersubjective communication and still directed the child through tasks, although it is not congruent with 
Vygotsky's theory of cognitive development $(1978,1986)$. Guided participation is a process, an approach to teaching that values the children, their backgrounds, and their interests, thereby giving children the wheel to drive their own learning.

I believe there is widespread misunderstanding of what Vygotsky considered communication and child-centered pedagogy (this is the modern term to a concept that was not 'named'). Refer to Ann as she talked to her children about the baby apple tree. She did not show them the roots, stem, and leaves and ask the children to repeat what she said. Ann referenced the children's previous experiences with that same tree growing outside before she brought it in to examine and before the children were involved in planting it outside in a more permanent location. She engaged the children by showing her own interest and curiosity (emotions) and sense of wonder at the function of the roots, and how apples will grow on it when it gets bigger. The children received her emotional (think sincere, authentic) communication on the concept of a baby tree, along with the actual tree, as Ann scaffolded their deeper understanding of trees. Scaffolding involves context, and in this case, she can eventually make greater connections such as fruit trees, other types of trees, forests, habitats, and so on, indefinitely. Children in Ann's class talked with each other socially and while talking about the tree, or the seeds, or the plants that were being planted together in one big container.

In contrast, and throughout their interactions with children, Denise and KA stayed true to direct instruction. I found the children in their classrooms unable to effectively interact with each other. Denise worked one-on-one both days and indicated that is her usual method of instruction when 'work' is done. Children in her classroom do not have a chance to collaborate in their learning. KA had a group while she instructed Zoo Phonics, although they were directed to pay attention to her. Diversions were admonished as she reminded children to "look at me," or "come 
sit over here." Without checking a child's prior knowledge, or communicating intersubjectively, concepts covered in Denise's and KA's classrooms, patterns, farm, phonics, (letters and letter sounds) were presented through instruction by definition, or by reviewing what is already known. Denise indicated that her children were introduced to patterns before. On the first observation day, video data indicates she instructed by definition, which is AB patterning. She told the children what to do rather than let the children uncover patterns through playful learning. The same holds for KA, who did not participate with the children and guide them toward recognition and sounds of letters through playful learning. Vygotsky addressed these practices:

Two important drawbacks make this method inadequate for studying the process in depth. In the first place, it deals with the finished product of concept formation, overlooking the dynamics and the development of the process itself. Rather than tapping the child's thinking, it often elicits a mere reproduction of verbal knowledge, of readymade definitions provided from without. It may be a test of the child's knowledge and experience, or of his linguistic development, rather than a study of an intellectual process in the true sense. In the second place, this method, concentrating on the word, fails to take into account the perception and the mental elaboration of the sensory material that give birth to the concept. The sensory material and the word are both indispensable parts of concept formation. Studying the word separately puts the process on the purely verbal plane, which is uncharacteristic of the child's thinking (Vygotsky, 1986, pp. 96-97). Denise remained directive with limited, i.e. obvious choices for patterns when she engaged children in $\mathrm{AB}$ patterns and the KWL chart. On both days, when a child did not respond to Denise's prompt, she gave them suggestions that were answers. For example, when she asked what you see on a farm, a few children did not respond. She offered, "Do you see chickens on a 
farm?" The child nodded, and Denise wrote that as the child's response and went on to the next question. KA remained directive, controlling the group working on phonics and with the single child finding matches to letters. A salient note of KA's direction was her slightly surprised look (wide eyes, mouth shaped as if to say 'oh') and statement that began with "I wonder," or "I have an idea." There were obvious or no choices for either Denise or KA's students. Ann was fluid in her differentiation, as she spoke to each individual child in the course of addressing all the children. For example, on the second observation day Ann guided the children to place baby plants in a very large planter. Ann softened her tone and gently encouraged a slow to warm up child to make a hole when it was her turn. Ann knew the child, so she first addressed a more outgoing child to get his plant in the large pot. Knowing the child would shake her head 'no,' Ann asked if someone else should go first. The child nodded and Ann had the others take their turn. Ann's knowledge of the child and her response to that child's individual needs, let the child know she is encouraged to take a turn, and gave her time to observe others and see how they responded to the experience. Illustrating the social dynamic of concept development, another child left the group to get gloves for this child because he knew she was not comfortable getting things on her hands, such as soil.

The children in Denise and KA's classrooms did what they were told. There was no real sense that the children understood the concepts of phonics, the farm, or patterns. There was no conclusion, no acknowledgement of the work that was done. Children left the instruction, having been dismissed, and returned to their previous, unrelated play. If there was evidence of learning, it would be by rote memorization or guessing. I found the children in Ann's class engaged in social interaction as well as instructional interaction. They talked with Ann as a member of the group, and she did not require the children to stay with her. Ann and the children were making 
discoveries together. She gave them the opportunity to explore and investigate concepts based on what they indicated was of interest to them. Ann stimulated curiosity and listened more than she spoke. Denise directed children to complete a task. KA directed children to listen to her.

I reviewed video of each classroom environment for evidence of intersubjective communication in that context. All three teachers displayed children's work as well as photos of children and families (home) in a designated space. There was no connection between home and what learning was displayed in any of the three classrooms. For example, Ann shared that her students became interested in growing plants and gardening from two angles; first were the spring growth of plants observed in the playground, and second, one child's grandmother had him engaged in weeding the garden. If these catalysts were recorded in photograph or other media and displayed in the physical environment, that would have deepened the intersubjectivity of the concepts being investigated.

Chapter five provides a summary of the three research questions in this study, in addition to a comparison of this work to previous research. Limitations of the study will be discussed along with implications for practice in early childhood education. The final section will offer reflections about optimal preschool pedagogy in high quality environments. 


\section{CHAPTER V: DISCUSSION}

Early childhood education and child development textbook authors recognize the value and importance of Vygotsky's sociocultural theory of cognitive development. Wherever it is discussed in text, the concepts of scaffolding and ZPD are prominent, and mention the importance of 'interaction' while scaffolding knowledge (Berger, 2018; Kostelnik et al., 2015). However, there is little explanation of learning as concept-building, and the importance of multimodal communication before and during scaffolding. There are misinterpretations of Vygotsky's theory as in Kostelnik et al. (2015). Mayesky (2011) goes to great depth to support teachers in child-directed pedagogy and does not express the importance of dialogue or conversation other than touching on open-ended questions and getting to know the children's interests. Conceptbuilding and multi-modal communication needs more attention in early childhood programs, texts, and teacher education programs. This study was an effort to support that need.

This study was designed to begin a dialogue among professionals, faculty, and policymakers. Investigating intersubjectivity among teachers and children during instruction in three preschool classrooms was the purpose of this study. Findings indicate that two of the three teachers, Denise, and KA, used more direct instruction and Ann used guided participation. The two teachers using direct instruction did not engage children in open-ended conversations or reference their backgrounds (culture) to make meaningful connections to the concepts of instruction, suggesting they did not communicate intersubjectively. Evidence also seems to indicate it is unlikely that the children expanded their concept development in Denise and KA's classrooms. 
In this chapter, a summary of the three research questions compared to extant literature on this topic and Vygotsky's cultural-historical theory of cognitive development is provided. Limitations and implications for research and practice will also be discussed.

\section{Guided Participation/Mediated Activities}

This study focused on investigating intersubjectivity - mutual understanding - reached by way of the communication between preschool teacher and child, specifically in relation to instruction in early childhood environments. Guided participation/mediated activity is explained herein as the process involving verbal and non-verbal communication, and the use of materials between teacher and child. Using these tools, a teacher engages in conversation embedding the background of both interlocutors so they may reach mutual understanding of each other's prior knowledge. Once the teacher determines they both understand each other, she reaches the point whereby they can enter the zone of proximal development (ZPD). In the ZPD, the teacher will maintain intersubjective discourse and demonstration, and continue guided participation, mediating the child's concept knowledge in deeper contexts.

Vygotsky's theory distinguishes between natural function and cultural function (Kozulin, 1986). Natural function is the brain remembering, memorizing, concepts based on repetitions. "According to Vygotsky, human higher mental functions must be viewed as products of mediated activity [guided participation]. The role of mediator is played by psychological tools and means of interpersonal communication" (Kozulin, 1987, p. xxiv). Based on interviews and observations, Denise and KA repeated concepts to children without intersubjective communications. They directed what the children experienced during instruction. Based on interviews and observations, Ann mediated activities with children. Ann and the children engaged in interpersonal communication - talking with each other, teacher to child, child to 
child, using gestures, and interacting with materials. Experiences are processes that facilitate higher functioning in which the children mediated their own concept knowledge based on multimodal communication. On this, Vygotsky (1978) wrote,

An interpersonal process is transformed into an intrapersonal one. Every function in the child's cultural development appears twice: first, on the social level, and later, on the individual level; first, between people (interpsychological), and then inside the child (intrapsychological). This applied equally to voluntary attention, to logical memory, and to the formation of concepts. All the higher functions originate as actual relations between human individuals (p. 57).

While determining what information to gather from Denise, KA, and Ann, I found it important to learn the participants' awareness of guided participation/mediated activity. Being cognizant that required professional development training may have informed the participants of practices without connecting them to the terminology, interview questions offered opportunities for them to explain practice even if they did not know the terms.

Effective guided participation relies on a teacher's knowledge of children's backgrounds and relating those backgrounds, using verbal and non-verbal communication with materials, during instruction (Vygotsky 1978, 1986). I asked participants to describe their style of teaching, whether it is more child-directed or teacher-directed. The intention was to gauge teachers' awareness of their role in influencing children's mediation of cognitive development. This speaks to the social and meaning-making aspects of intersubjectivity (Fultner, 2012).

Next, I moved into communication, and asked for descriptions of ways in which Denise, KA, and Ann communicated with children, especially during instruction. Evidence suggests that Denise did not have knowledge of guided participation, as she maintained a straightforward, 
teacher-directed approach to learning. During her first interview, KA used the terms 'coteaching', 'facilitating,' and 'open-ended questions,' that offered possibilities that she utilized intersubjective pedagogy. Nevertheless, the observations and follow-up interview indicated obfuscation of those concepts. Ann approached guided participation and mediated activity as two separate concepts indicating some misunderstanding. However, she was familiar with the general idea by describing, "guiding the child in the direction that you want them to go." She was clear about child-directed teaching and demonstrated that in observations as well as in our follow-up interview.

\section{Intersubjectivity and ZPD}

Research questions two and three were aimed at intersubjectivity to first assess prior knowledge of concepts and readiness to enter the ZPD, and second, mediating the same concepts in the ZPD using communication that includes meaningfully relating the concepts to a child's background knowledge (culture). Referring again to Bodrova and Leong (2007), I ask that readers consider the term 'guided participation' in place of 'scaffolding,' and the three ideas relevant to the phenomenon. First, teacher support will vary with each child and is never to make the task easier. Also, the teacher will yield responsibility of the performance to the child as she demonstrates understanding. Lastly, the teacher recognizes her intervention is temporary and, in response to the child's ability and understanding, will slowly back away from supporting the child as she becomes independent in her problem solving. This process of guided participation has no finite end as the relationship between teacher and child will influence effective instruction. Intersubjectively, Vygotsky $(1978,1986)$ contends that children will understand best when demonstration (that may include materials) accompanies verbal discourse, presented concurrently and in a meaningful context, using a play-learning approach. 
Responses to teaching style and background knowledge provided evidence of participants' perceptions about mutual understanding. In addition, the three preschool teachers were asked to describe and explain the verbal and non-verbal methods of communication they used during instruction. The next set of interview questions asked what information teachers sought to determine a child's readiness for instruction.

The culminating inquiry at our first interview made an impact on me. It read, 'How do you know whether a child understands you?' In other words, did the preschool teacher and her children reach intersubjectivity? In our interview, Denise spoke of letter identification and letter sounds in teacher-directed way. "For example, we're talking about where the letter is... what the letter represents, how to sound it out and what letter is in your name. And 'b' sounds like 'buh,' so we're pronouncing your name now." This example, with the preponderance of observational evidence, and our follow-up interview led me to conclude that, during this study, Denise practiced teacher-directed pedagogy without clear knowledge of mutual understanding or how to assess a child's understanding. Denise directed her knowledge to children. KA recognized that understanding could come from demonstration of knowledge, as she stated, "I've set up something. I've encouraged it. I've exposed them. I've offered repetition and practice and then they're going out and spitting it back out. That's when I know they truly understand." In the case of KA, the evidence gathered during the study leaned heavily toward a teacher-directed pedagogy. She indicated a possible understanding of child-directed learning by using the concepts 'facilitation' and 'co-teaching' in context. However, even in describing a child's understanding in the quote above, she uses 'I' and not 'we,' or 'the children.' It appears, therefore, that KA also directed instruction to the children intending them to accept the material 
presented using drill to skill pedagogy. Ann shared a different explanation as to how she knows children understand her:

A lot of people think [children] are supposed to get things right away. No. You have to keep repeating it in different ways and find different ways of doing it. Once you find the way that works for the child, you'll see it. You will see it on their face like, 'oh, that's what you meant.

Findings show that Ann joined the children in their learning using materials and verbal and non-verbal communication so children could make meaningful connections of the concepts presented. She collaborated with the children during instruction, assessing their prior knowledge by reviewing what they previously shared and asking historical, open-ended questions. She then proceeded into the ZPD with the children to widen and deepen their conceptual understanding of plants, whether they are trees or flowers, using materials for context. Like the teacher in the Gjems (2013) study, as the children mediated their knowledge, Ann was present to acknowledge, review, use actual materials, and ask open-ended questions. Then, whether a child made a meaningful connection to a concept that day or not, Ann would revisit the progress made that day on the next school day, and build from there, fluidly moving back and forward with each child through their learning trajectory.

This study focused on intersubjectivity, and surrounding this concept is guided participation. Within the concept are vital elements - interrelated concepts - that were realized during interviews and two observations with each preschool teacher. Concepts connected with intersubjectivity are guided participation/mediated activity, the ZPD, and concept development. Referring to Figures 1 and 2 in chapter one may offer a better understanding of the relationship 
between these concepts. Vygotsky $(1978,1986)$ described effective teaching that, during instruction, takes place outside an intangible place (the ZPD) as well as within the ZPD.

Literature on guided participation found various terms that define the same thing; mediated activity, guided participation, and pedagogical psychology were used by Vygotsky (1978, 1986, 1997a, 1997b), who discussed these as higher mental processes that "depend on the presence of mediating agents in the child's interaction with the environment. Vygotsky himself primarily emphasized symbolic tools - mediators appropriated by children in the context of particular sociocultural activities, the most important of which he considered to be formal education" (Kozulin, 2003, p. 17). Wood et al. (1976) and Wood (1999) interpreted and developed these concepts as akin to tutoring and adopted the term 'scaffolding.' According to Kozulin (2003), Vygotsky's view of guided participation is an acquisition model whereby children mediate their knowledge. Scaffolding altered the process into a 'mediation model' (Kozulin, 2003) that alters guided participation (child-directed pedagogy) into tutoring (teacherdirected pedagogy). Thus, the term 'scaffolding' does not relate to Vygotsky's cultural-historical theory of cognitive development.

Guided participation requires teachers to intersubjectively engage children in communication, whether it be verbal or non-verbal, as well as the use of tools - words, gestures, materials - as aids in understanding (Leong \& Bodrova, 2007; Vygotsky, 1978, 1986). Using Denise's patterning instruction and applying it to Vygotsky's theory as an example, Denise would have worked with more than one child at a time (because learning is a social construct benefitting from MKOs). She may have used colored unifix cubes, or other materials to make patterns previously, at the table within reach of herself and a small group of children so she could give one-on-one instruction as appropriate. Denise may ask the children if they remember 
patterns and invite them to use the cubes to make one. Based on what Denise already knows the children to have experienced regarding patterns, she may see them making an AB pattern with more than two repetitions of each attribute. Perhaps she introduced children to AB patterns using colors with these same unifix cubes. Throughout the process, Denise would talk with the children about the last times they addressed patterns. As she actively participated, Denise may have lined up cubes in a certain way (e.g., ABAB) and mention how a child similarly lined up other counting pieces. She may observe and verbally note the pattern on a child's socks. These methods of communication reinforce the concept, 'pattern,' in context. The intention is to bring meaning of 'pattern' to a child's knowledge base by making connections and listening. The children who made an AB pattern independently are ready for the ZPD.

The ZPD is a difficult idea for many, as it is an intangible concept. Using a room as an analogy for the ZPD, effective teachers must begin instruction outside the ZPD, as Denise did in the previous example. Once prior knowledge is established, she will take the children through the door, into the ZPD.

Figure 4

Establish Prior Knowledge, Then Enter the ZPD

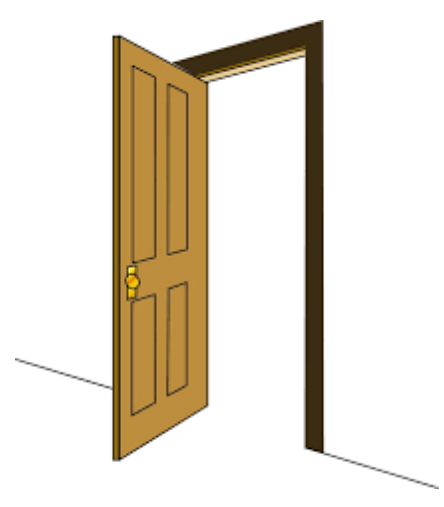

Using the observation of Denise's instruction on AB patterning using farm animals, here are thoughts on what she could have done to move children into the ZPD. Denise could bring out 
groups of different animals without disturbing the unifix patterns and make an observation such as, "I see the patterns you made with these colored cubes. I wonder if you could make a pattern using animals?" Instead of telling the children what to do, Denise would invite them to join her and interact with the animals and the concept that was presented - patterns. She would be part of the play and make a pattern herself to see if others notice. She may invite others to see what she did. Denise would respond to statements and questions from the children. She would refer to the children's background information connects them to the concept. If the children had very little or no experience with patterns more complex than $\mathrm{AB}$ color patterns, they are now in the ZPD. Extending this concept for those more capable, Denise would participate and encourage more complex patterns. In other contexts, she could guide participation in a more natural, playful way as children are interacting with blocks, manipulatives, crayons, or shoes, for example.

As described in Figure 2, Denise would support the children who need assistance without giving the answers, thereby encouraging the children to think about what pattern means in this context. She would use the materials to support meaning making for the children. One child may help another, verbally and by demonstration. The experience making patterns from animals as opposed to colors changes the context of 'patterns.' They should not be expected to 'get it' the first time they try. Denise would help the children connect the idea of 'every other one' with two animals to make an $\mathrm{AB}$ pattern interpsychologically. The children would always be able to choose to disengage from instruction to avoid becoming frustrated (Vygotsky, 1986). Denise would keep materials in the classroom where the children could explore patterns independently and with her invitation. She would assist children when they ask for help. The children would mediate 'pattern' on their own and, over time, Denise would see that and document the child's next level of concept development. Another day, she may determine/reinforce prior knowledge 
and take the children into the ZPD using patterns in another context, such as a growing pattern (e.g., The Napping House), ABC, or pointing materials up and down. Throughout the exploration Denise would be prepared to intersubjectively communicate a more complex pattern in response to the children's interest. In this example, children socially receive concepts, mediate them, and then transform the concepts intrapsychologically, to be internalized and later generalized.

My thoughts to illustrate how KA could have presented her letter sounds instruction to children using guided participation in the ZPD are as follows. To mediate a child's development of letter sounds, KA would have determined, through intersubjective communications over time that the children had prior knowledge of letter sounds in their name, for example. KA would join the children at the writing center where they are using crayons, pencils, and markers to make pictures. She would engage the children intersubjectively to learn about the motivation behind the child's work and engage in relational communication. KA would encourage a cultural practice by encouraging the child to write their name on the work. Then, during their engagement in writing, KA might say the sound of the first letter in the child's name. Some names will have blends to begin their name such as 'Sharon,' or 'harles.' Calling upon her training, KA would say their names are unique because it takes two letters to make /sh/ and /ch/. The children would remember these sounds more readily because they are personally meaningful to each individual. This example is not to be misunderstood as direct instruction while children are writing or drawing. KA would, in a sense, step in and out of the ZPD with the child. She would not continue to drill children on letter sounds; she would be intentional about making meaning for the child in that moment and leave the child to continue with their chosen experience. If the child engages KA further, she is there to guide them. 
Observing Ann in her classroom, I documented her mediating the concept of 'growth' in the context of plant growth. It was spring, and in previous weeks the children noticed new growth on their neighborhood walks, around their homes, and in the playground at preschool. She expanded their interest in this phenomenon and brought a baby apple tree into the room. Ann had an apple which she cut for everyone to taste and noted the seeds inside. A child mentioned that they had seeds like this another day. Ann acknowledged this and asked what they remembered about the seeds. A child said, "you plant them, and they grow." Ann facilitated a conversation with the children about things they have experienced, assessing prior knowledge. On that day, she had plants in the room for the children to plant in dirt. They had not done this before, although they all knew plants need dirt - and water and sun - to grow. She guided each child about scooping a hole in the dirt and helped if necessary, patting the soil around the plant, and watering each plant so it could settle in. This day, the children put plants into a large pot and took it outside to a location the children determined was best for the flowers to grow. Ann did not intervene in their placement, as it was to be a learning experience over time.

This fluid engagement expresses how Ann established the children's prior knowledge that seeds are needed for baby plants to start growing. She used the materials, her face, her voice, and gestures to engage in discourse. Ann looked to each for signs of acknowledgment and paused for the children's contemplation. Two different children commented on sorting the seeds, meaning they will make different 'flowers.' Her open-ended approach gave the children a natural path of thought and collaboration as one child indicated, with voice, expression, and gestures, the flowers had different seeds. The extended observations of the children are examples of them in the ZPD. If the other children did not voice their understanding, they are listening to their peers and learning from them as well. Because the ZPD is intangible, oftentimes there is no specified 
time or point that is easily recognized as children going in and out of the ZPD, and yet, that is exactly what happens. In addition, the ZPD is where levels of practice, contemplation, learning, internalization, and generalization happen. It is likely that every child is in the ZPD at a different stage of concept development even if the group is engaged in the same experiences.

\section{Limitations of the Study}

Although the findings from this study shed light on the use of intersubjectivity in preschool classrooms, the study was not without limitations. To find preschool classrooms most likely to illustrate the presence of this phenomenon, I sought preschool classrooms to include at least one whose teacher held a bachelor's degree in early childhood education (ECE). Level of education has become one of the premier criteria of high quality education, as indicated by NAEYC (2015), the Organisation for Economic Cooperation and Development (2016), and the Institute of Medicine and National Research Council (IOM \& NRC, 2015). With such emphasis on a baccalaureate degree, I wondered whether teachers with baccalaureate degrees in ECE are aware of and use culturally based communication strategies that will lead to mutual understanding. As such, this study included no evidence as to whether teachers with an associate degree in early childhood education (or other less formal training and/or education) engage students toward intersubjectivity in similar or different ways than the three teachers in this study.

The small sample size was also a limitation. In this study, the researcher focused on using case study methodology, which offered relative depth of information for participants rather than information on many teachers and children. Researching three participant preschool teachers offered a limited breadth of study, both in numbers and in geographical area. Given more time and added participants from a greater area, the results may affect implications of the study. More participants would add greater representation of AAS and BA degreed teachers. Time of year 
(spring 'semester') and time allotted to complete the research caused me to compromise the desired number of teachers in study. Once the data was evaluated, I presented findings from three participants: two with BA in ECE degrees, and one with an AAS in ECE.

Although rich data was gathered and shared, one qualitative research study about a particular portion of Vygotsky's cultural-historical theory of cognitive development such as this is not enough to drive practice, even if generalizations are presented. This investigation enjoyed interaction with each of three participants for two interviews and two observations. All three were offered a final, optional follow-up interview and all three declined.

Given the seven-to-ten-day span in which I saw the teachers for two to three total interview hours, and one-to-two observation hours, it was a very short time to develop a relationship that would offer a deeper, mutual understanding of their perspectives. It seemed at first to be ample time to get to know the teachers. However, terms were unfamiliar with all three, and time was a challenging factor. These generous teachers volunteered to see me during their busy days, for initial interview, observation one, observation two, and follow-up interview. It was challenging to get participants willing to be videotaped as they engaged children during instruction.

The case study method was chosen based on time constraints, availability of participants, and its appropriateness to study intersubjectivity. Intersubjectivity is the case, "defined as a phenomenon of some sort occurring in bounded context" (Miles et al., 2014, p.28). Case study gave me the opportunity to get to know participants, who themselves served as sub cases (Miles et al., 2014). A salient limitation to this method is that two of the three participants did not practice the phenomenon I investigated. 


\section{Implications for Future Research}

Limitations of this study, as discussed, provide rationale to drive future research toward alternate methodologies. Evidence in this study indicated that Ann practiced guided participation with her students. Future research offers possibilities of case study where guided participation is practiced by all participants. Looking back at this work, investigating intersubjectivity, future investigations might concentrate on teachers who practice guided participation. Engaging in research over a school year would yield greater depth of study. Expanding the length of study would offer more data, revealing, perhaps, that a different week or unit of study might have changed the tenor of a case, both from the interview and observational perspectives. Another consideration for a longer study is that terminology presented to teachers in this study were unfamiliar. Subsequent studies in a variety of settings could help establish a comprehensive definition as well as identify program models that embrace intersubjective pedagogy. Given more time to develop intersubjective relationships with the teachers may also offer stronger results. Reaching intersubjectivity takes more time compared to direct instruction, and so more observation with supportive interviews and surveys lends itself to exploring this subject. Looking back at Ann's conversation with a child about play dough, we realize it took a while for him to express meaning, and her to understand that (through clarifying communication) he choked on it at home.

Taking a deeper dive into the elements and benefits of guided participation is an appealing prospect for future research. I plan to shine a light on the emotional aspect of adultchild intersubjective communication. In chapter four of Thought and Language, Vygotsky (1986) connected emotion to thought and speech of primates to humans, who developed a more complex mechanism for these functions. In later years, Foolen (2012) and Lüdtke (2012), among 
others, related emotion to language development and intersubjectivity. I believe these developmental dynamics have a strong impact on early childhood education and will benefit being brought to the attention of every ECE professional via conferences, courses, journal articles and teacher education programs.

Future Research to Expand Standards and tools relating to high quality ECE professionals

Vygotsky's cultural-historical theory can be found in early learning and development standards (IELDS), child development textbooks (e.g. Berger, 2018; Berk, 2021; Morrison, 2018) and the Classroom Assessment Scoring System, commonly referred to as CLASS (Pianta et al., 2008). Still, there is very little, if any, mention, or training in intersubjective communications as part of guided participation. For example, the IELDS (2013) indicate certain behaviors and skills children between three and five years of age are typically able to demonstrate 'with teacher assistance,' such as, "With teacher assistance, identify main character(s) of the story" (2.B.EC.c). This indicates a child will likely be in the ZPD until the concept of main characters in stories becomes internalized, then generalized. Future research to validate this and other approaches to children's development may lead to a review of the IELDS, which have not been revised since 2013 .

The CLASS is a perfect forum for guided participation. Pianta et al. (2008) wrote, "The CLASS focuses on interactions between teachers and students and what teachers $d o$ with the materials they have." It is an observation tool rather than an instructional tool which may justify the lack of explanation regarding effective interactions. Head Start requires the CLASS to be administered in their classrooms annually and monitors scores of their teachers. Their message to teachers includes: 
Effective, engaging interactions and environments form the foundation for all learning in early childhood classrooms. These high-quality preschool practices include a wellorganized and managed classroom, social and emotional support, and instructional interactions and materials that stimulate children's thinking and skills. Such interactions involve the back-and-forth exchanges among teachers and children that occur every moment of the day (Head Start ECLKC, 2013, p. 2).

Note the recognition that effective interactions are foundational, and that they involve the back and forth exchanges as critical teaching skills. More inquiry is needed to see if teachers are getting the resources they need to understand the 'why' and 'how' to be more effective educators.

Based on the findings, implications for further research includes expanding CLASS (Pianta et al., 2008) to include a) deeper contexts of interactions referencing a child's home life or the child's personal experiences, b) being intentional about supportive learning materials, c) using expression, tone, gestures to enhance verbal communication, and d) how to determine mutual understanding in the moment and over time. Communicating - guided participation - is a process rather than a task. Looking at the national CLASS scores from 2019 (Head Start ECLKC, 2020), the latest year reported, instructional support was the lowest scoring domain. Within that domain, the dimension of concept development showed the lowest scores, from a minimum score of $1.44 / 7.0$, and the highest score being 4.0/7.0. The mean score is 2.43 out of 7.0. Also on Head Start's website is a page on Teaching Practices (2020) with supportive information to improve concept development. Instructions include facilitating creativity, and higher order thinking. Information addressing 'how' to facilitate learning is missing. Teachers may know what to do and not know how to make it happen. Professional development and 
embedding this information in higher education coursework may be an asset to teacher education development. Quantitative information in the form of ECERS (Harms et al., 2015) and CLASS (Pianta et al., 2008), currently administered in Head Start classrooms may offer interesting correlations of intersubjectivity to social, emotional, and cognitive preschool learning outcomes.

\section{Future Research Methods}

After completing this study, it occurs that investigating intersubjective pedagogy using action research would be an interesting and informative approach. It is an interpretivist method that works in phases that include observing, reflecting, and acting (Kemmis \& McTaggart, 1988; Stringer, 1999). Utilizing an action research approach, researchers could use qualitative interviews and observations (as this study utilized and add surveys and quantifiable data with the potential to venture into mix-methods research. Unique to action research is the reflection and action cycles whereby researchers interpret data already collected, inform all participants (that may include administrators), referred to as stakeholders (Glesne, 2011), discuss action to be taken, and then act. "The research process is collaborative and inclusive of all major stakeholders with the researcher acting as a facilitator who keeps the research cycles moving" (Glesne, 2011, p. 23). What makes this an exciting prospect is that it lives past outcomes, through implications, and on to another phase of research.

Action research is a method seemingly tailor-made for studying intersubjectivity and guided participation due to its potential to be agents of change. Reiterating that guided participation is not a familiar term with teachers, those who follow elements of this pedagogy, such as Ann, may be enlisted to learn as they participate. In one application, researchers who are insiders to the community in which they study can work alongside other stakeholders to strengthen the concept, depending on its affordances. "Insiders who couple research theories and 
techniques with an action-oriented mode can develop collaborative, reflective data collecting and analysis procedures for their own practices and thereby contribute to the sociopolitical context in which they dwell” (Glesne, 2011, p. 23).

In a recently published editorial on the value of educational action research for students in higher education, Norton and Arnold (2021) cite research from Europe and the United States, suggesting benefits of pedagogical action research (PedAR) for higher education, professionals, and communities. For example, teacher communities would benefit from developing their pedagogical skills, mentoring teacher candidates, and mentoring early career teachers. Given the critical state of early childhood education in the United States and the lack of high quality child care as we begin recovery from the Covid-19 pandemic, improving child care through pedagogical action research influenced by community-driven, collaborative efforts may provide an attractive method of developing sustainable quality child care. Larger, comprehensive studies that follow children's progress throughout a preschool year, such as action research with mentoring of teachers, would add trustworthiness of the study (Glesne, 2011) or what Saldaña calls the "That's right!" factor (Miles et al., 2014).

\section{Implications for Practice}

Of the three teachers who participated in this study, Ann is the one who demonstrated knowledge of the theory and practice of guided participation and intersubjective communication. After the data collection phase of the investigation concluded, I asked Ann how she developed her pedagogical philosophy. Her response was that she is self-motivated to keep up on responsive teaching and gravitates toward the Reggio Emilia approach to teaching and learning. She admitting there is not a lot of training available in this area geographically or topically. 
I believe there are. Given that Vygotsky's theory influences pedagogy, program, and professional assessments, e.g., CLASS (Pianta et al., 2008) and ExceleRate Illinois (2017), and professional preparation standards (NAEYC); and given that intersubjectivity specifically pertains to mutual understanding reached through multi-modal communication, the implication that the concept belongs in professional development and teacher education programs deserves consideration. Professional development through mentoring and coaching, conferences, inservice trainings, in published works and in higher education teacher education programs are opportunities to share this knowledge.

\section{Implications for Professional Assessments and Preparation Standards}

Progressing through higher education teacher education programs, students are likely to be introduced to NAEYC 2020 Professional Preparation Standards, as most textbooks reference their content to the newly named and developed key competencies (formerly key elements). If the early childhood education community looks to NAEYC and their professional standards and competencies for quality teaching and learning, we should see more teachers like Ann, who demonstrated developmentally appropriate practices (DAP) by nurturing the children's innate curiosity and guiding participation through play. Using the principles of emergent curriculum and communicating in the style of conversation, Ann believes her responsibility is

to make sure all of the children are learning in the way they need to learn. Some are auditory learners, some are visual, some are kinesthetic. Some children don't want to be touched, and if you talk to them, they're happy with that.

As noted, the standard for quality programs and developmentally appropriate practices (DAP) has been set by the National Association for the Education of Young Children (NAEYC), the largest early childhood educator membership organization in the United States. NAEYC 
offers conferences, two journals, a plethora of publications and professional development opportunities. The evolution of DAP is based on solid research and practice over many years with resources and application available on NAEYC's website (NAEYC, n.d.).

While Vygotsky is not the sole contributor to DAP by any means, there are important aspects of his work that are reflected in them, such as child-centered learning, play-based pedagogy, collaborative learning (MKOs), and being responsive to the abilities and cultures of children and their families. Perhaps the concept of 'understanding,' especially 'mutual understanding,' has been overlooked, or considered a 'given.' That implies that systems of training, education, research, may have considered that teachers 'naturally' reach mutual understanding before concept development can proceed. Perhaps it has been overlooked. This happens to our professional detriment, when theoretical constructs are fragmented and parts of the whole are used. It tends to be our professional habit to forge a clear and easy path for educators by using catchy phrases or ideas that can be adopted by early childhood teachers quickly.

\section{Implications for Professional Development and Teacher Education Programs (TEPs)}

Based on these theoretical dynamics of learning, there seems to be a strong case to embed Vygotsky's theory to a greater extent - especially discussions on guided participation/mediated activity, its process, and developmental goals - into TEPs and professional development systems. Even though he is considered a pillar among education theorists, many parts of the cultural-historical theory of cognitive development are lost in translation (Gonzalez Rey, 2008) in the western world. Intersubjectivity, the concept being explored in this research is an aggregate of many concepts that include emotion, communication, sense, meaning, and subjectivity. Within these concepts, sense and subjectivity are themselves complex psychological 
phenomena. Learning and understanding these dynamics brings teaching further away from direct instruction and closer to teaching and learning as a social and psychological construct.

Consider a learning opportunity in which pre- or in-service teachers watch videos or learn from scenarios where teachers utilize intersubjective instruction appropriately. For example, learners would witness teachers approaching learning as a participant with the children. She will have established the environment with children's physical perspective in mind, to invite the curious to active engagement with open-ended, including natural, learning materials. Before participating in play, the teacher will observe the children to get a sense of their cognitive plan. Are they building with blocks? Are they seeing how high they can stack blocks, making a castle, zoo, or neighborhood? When she joins the children in their activity, as another curious participant, she will not direct the play, she will be a more knowledgeable other $(M K O)$, embedding observations, wonderments, and open-ended questions, using multi-modal methods of communication. In other words, she may use gestures, facial expressions, tone of voice, other materials, and words to engage the children, as one of the group.

Observations continue and learners would see that the initial purpose of communication is to learn the children's current perspectives and knowledge. The teacher fluidly assesses one or more children's current concept development to determine whether she will support their current level of concept development or expand on it to another context. Without the benefit of a physical doorway, the teacher takes one or more children into the ZPD. How long will she stay with any given child is for both MKO and child to determine, with the greater responsibility on the teacher to avoid causing frustration. Maintaining intersubjective communication throughout, the teacher observes and communicates to determine when to leave the child to her own learning. The child may stay in the ZPD for a moment or a while, exiting when they choose. Instructors 
can lead critical analysis and provide opportunities for participants to practice this dance. Furthermore, for in-service teachers, mentors, and coaches would work with them in the classroom setting to comment on the learners' ability to embed intersubjectivity into guided participation, taking children in and out of the ZPD.

Additionally, instructors, mentors, and coaches should help pre- and in-service teachers better understand that the reasons why facial expression, tone, and gestures are critical in reaching intersubjectivity are that emotion is at the core of communication, starting at birth. Throughout our lives we communicate with emotion, whether it is easily labeled happiness and sadness, or the less obvious interest or trust. Vygotsky (1986) included emotion in his theory because of its contribution to concept formation. Vygotsky believed that verbal requests of children to elicit concept knowledge "often elicits a mere reproduction of verbal knowledge, of ready-made definitions provided from without" and that "concentrating on the word fails to take into account the perception and mental elaboration of the sensory material that gave birth to the concept" (p. 96). Therefore, "the sensory material and the word are both indispensable parts of concept formation" (p. 97). Instructors, mentors, and coaches could offer opportunities to observe guided participation in videos or in the field. Reflecting on the experiences verbally and in writing would help determine the learners' levels of understanding to inform instruction. Instructors should also engage in multi-modal communication as well, practicing what she teaches, so she and the learners will reach intersubjectivity.

To put this in the context of the study, the children being instructed by Denise and KA either did what they were told, did not do what was asked and got answers from their teacher, or they did not participate (in a group setting) and may or may not have internalized knowledge. Ann practiced a different pedagogy. She actively engaged each child with verbal and non-verbal 
communication along with materials to investigate. Ann was an active participant in the learning - joining the children and learning with them and making observations and discoveries together. The children asked questions, spoke to each other and Ann, and they were not limited to the academic content she shared. Comparing the experiences of Ann's students to those of Denise and KA was striking. Ann's students demonstrated inquiry, laughter, interest in the material and in each other, while children in the other classrooms did not get those opportunities during instruction.

This evidence leads me to believe there is promise in expanding content of Vygotsky's cultural-historical theory in higher education teacher education programs, especially at the point of field experiences and student reflections. There are many opportunities for students to observe non-examples of guided participation. While they are powerful learning tools, there must be efforts to offer appropriate examples to future and practicing teachers. Observing in classrooms vetted for practices in guided participation, such as Ann's, students will see the benefits of guided participation in the delight of children exercising their innate curiosity in directions they choose. Those reactions to learning will reinforce teacher candidates to continue practicing intersubjectively, rewarding children and adults with deeper understanding of each other.

\section{Concluding Reflections}

Do we need another term for guided participation, also known as mediated activity? Throughout this study, the question often rose in my mind, along with the wonderment that is, 'what is it about these terms that did not resonate with researchers of Vygotsky's theory over time?' I decided to suppress these questions in favor of the path chosen by Fernando Gonzalez Rey (2008) and other Vygotsky followers, which is, to stay as true to Vygotsky's work as

possible and expanding it with research. Introducing new terms and phrases muddies the 
concepts put to paper by a brilliant researcher at an historical time in Russia that makes his work valid today. I was tempted to explain guided participation using another term; to use 'intersubjective pedagogy' during which teachers engage in authentic, open-ended discourse, references to individual children's backgrounds (culture), and material supporting concept development, in a small group environment. My thoughts continued to explain that engaging children in this way first provides the teacher with an assessment of the children's prior knowledge. Once established, the children are 'taken' into the ZPD where 'intersubjective pedagogy' [i.e. guided participation] continues and expands to introduce new concepts or different contexts of concepts. As the children interact in this environment, using teacher and peers as MKOs and materials to support concept development, they mediate their own knowledge. MKOs are there to support each child as they construct the concept and, as they internalize the concept the MKOs are called upon less and less. When the child becomes independent in their concept knowledge, they will be able to generalize the concepts in other contexts (Kozulin, 1986; Vygotsky, 1986).

I choose to resist the propensity of some members of America's scholarship culture to reinvent terminology, thereby fragmenting important theoretical constructs to their detriment. I will continue the use of guided participation and its benefit in the context it was studied and continues to be studied today. I met Vygotsky via studies many years ago, and I have been fascinated with his work ever since. I see him in preschool environments where teachers like Ann listen and offer opportunities for children to investigate many things, as they are naturally curious to learn. I see him in environments wonder-filled with grand possibilities; where teachers are ready to assist and support children through behaviors and then step back to let the child engage on their terms, returning to assist if necessary and again retreat. I see him where children 
arrive with bright faces, eager to be detectives, artists, musicians, writers, builders - anything they wish to be that day.

Currently, ECE is a fragmented discipline and children deserve better. If the idea of intersubjective communication, to be the interactive method of communicating with young children, takes hold, our children will be better positioned to develop critical thinking. I see this as a merge of social-emotional attention and learning if teachers do not try to rush through a set of learning benchmarks that often give a false sense of accomplishment, as witnessed in some of the classrooms in this study. Imagine the possibilities for critical thinking. 


\section{REFERENCES}

Abtahi, Y., Graven, M., \& Lerman, S. (2017). Conceptualising the more knowledgeable other within a multi-directional ZPD. Educational Studies in Mathematics, 96, 275-287. https://doi.org/10.1007/s10649-017-9768-1

Berger, K. S. (2018). The developing person through childhood ( $8^{\text {th }}$ ed.). Worth.

Berk, L. E., \& Meyers, A. B. (2021). Infants and children: Prenatal through middle childhood ( $9^{\text {th }}$ ed.). Pearson.

Berk, L. E., \& Winsler, A. (1995). Scaffolding children's learning: Vygotsky and early childhood education. National Association for the Education of Young Children.

Bodrova, E., \& Leong, D. (2005). High quality preschool programs: What would Vygotsky say? Early Education and Development, 16(4), 437-446.

Bodrova, E., \& Leong, D. (2007). Tools of the mind: The Vygotskian approach to early childhood education ( $2^{\text {nd }}$ ed.). Prentice Hall.

Brinck, I. (2008). The role of intersubjectivity in the development of intentional communication. In Zlatev, J., Racine, T. P., Sinha, C., \& Itkonen, E. (Eds.). The shared mind: Perspectives on intersubjectivity (pp. 115-140). John Benjamins.

Bruner, J. (1990). Acts of meaning. Harvard University Press.

Buysse, V., Winton, P. J., \& Rous, B. (2009). Reaching consensus on a definition of professional development for the early childhood field. Topics in Early Childhood Special Education, $28(4), 235-243$.

Cresswell, J. W. (2007). Qualitative inquiry and research design (2 ${ }^{\text {nd }}$ ed.). Sage Publishing. 
Chadwick, C. (2015, October). Early childhood workforce in Illinois. In Illinois Education Research Council (IERC) $13^{\text {th }}$ annual meeting. [Symposium] IERC, Lisle, IL, United States.

Child Care Center U.S. (n.d.). Find a quality child care center. https://childcarecenter.us/state Creswell, J. W. (2007). Qualitative inquiry and research design (2 ${ }^{\text {nd }}$ ed.). Sage Publishing. Dahlberg, G, Moss, P, \& Pence, A. (1999). Beyond quality in early childhood education and care: Postmodern perspectives. RoutledgeFalmer.

Daniels, H. (2001). Vygotsky and pedagogy. RoutledgeFalmer.

Daniels H. (2014). Vygotsky and dialogic pedagogy. Kul'turno-istoricheskaya psikhologiya= Cultural-historical psychology, 10(3), 19-29.

Early, D. M., Maxwell, K. L., Burchinal, M., Alva, S., Bender, R. H., \& Bryant, D., Cai, K., Clifford, R. M., Ebanks, C., Griffin, J. A., Henry, G. T., Howes, C., Iriondo-Perez, J., Jeon, H., Mashburn, A. J., Peisner-Feinberg, E., Pianta, R. C., Vandergrift, N., \& Zill, N. (2007). Teachers' education, classroom quality, and young children's academic skills: Results from seven studies of preschool programs. Child Development, 78(2), pp. 558580. doi: 10.1111/j.1467-8624.2007.01014.x

Edwards, C., Gandini, L., \& Foreman, G. (Eds.). (2012). The hundred languages of children: The Reggio Emilia experience in transformation (3rd ed.). Praeger.

English Oxford Living Dictionary. (2017). https://en.oxforddictionaries.com/definition ExceleRate Illinois. (n.d.). ExceleRate Incentives for Center Based Programs. http://www.excelerateillinoisproviders.com/benefits/incentives/licensed-center-incentives ExceleRate Illinois. (n.d.). Program Overview. http://www.excelerateillinoisproviders.com/overview2/overview 
ExceleRate Illinois. (2017). Quality Standards Overview.

http://www.excelerateillinoisproviders.com/docman/resources/13-overview-of-charts/file

ExceleRate Illinois. (n.d.). Standard and Evidence Requirements.

https://www.excelerateillinoisproviders.com/resources/standard-and-evidencerequirements

ExceleRate Illinois. (n.d.). What is ExceleRate Illinois?

https://www.excelerateillinois.com/about/what-is-excelerate-illinois

Flint, T. K. (2010). Making meaning together: Buddy reading in a first grade classroom. Early Childhood Education Journal, 38, 289-297. doi: 10.1007/s10643-010-0418-9

Foolen, A. (2012). The relevance of emotion for language and linguistics. In A. Foolen, U. M. Lüdtke, T. P. Racine, \& J. Zlatev (Eds.), Moving ourselves, moving others: Motion and emotion in intersubjectivity, consciousness and language. John Benjamins, Amsterdam, The Netherlands.

Fredriksen, B. C. (2010). Meaning making, democratic participation and art in early childhood education: Can inspiring objects structure dynamic curricula? International Journal of Education Through Art, 6(3), 381-395.

Fultner, B. (2012). Intersubjectivity in the lifeworld: Meaning, cognition, and affect. In A. Foolen, U. M. Lüdtke, T. P. Racine, \& J. Zlatev (Eds.), Moving ourselves, moving others: Motion and emotion in intersubjectivity, consciousness and language. John Benjamins.

Fumoto, H. (2011). Teacher-child relationships and early childhood practice. Early Years, 31(1), 19-30. 
Gallagher, S., \& Hutto, D. D. (2008). Understanding others through primary interaction and narrative practice. In J. Zlatev, T. P. Racine, C. Sinha, \& E. Itkonen (Eds.). The shared mind: Perspectives on intersubjectivity. (pp. 17-38). John Benjamins.

Geertz, C. (1973). The interpretation of cultures. Basic Books.

Gjems, L. (2013). Teaching in ECE: Promoting children's language learning and cooperation on knowledge construction in everyday conversations in kindergarten. Teaching and Teacher Education, 29, 39-45.

Glick, J. (1997). Prologue. In R. W. Rieber (Ed.). The collected works of L. S. Vygotsky: Volume 4, the history of the development of higher mental functions (pp. v-xvi). Plenum Press.

Glesne, C. (2011). Becoming qualitative researchers: An introduction $\left(4^{\text {th }}\right.$ ed.). Pearson.

Gonzales-Rey, F. (2008). Subject, subjectivity, and development in cultural-historical psychology. In B. van Oers, W. Wardekker, E. Elbers, \& R. van de Veer (eds.). The transformation of learning: Advances in cultural-historical activity theory (pp. 137-154). Cambridge University Press.

Gredler, M.E., \& Shields, C. C. (2008). Vygotsky's legacy: A foundation for research and practice. Guilford Press.

Groenewald, T. (2004). A phenomenological research design illustrated. International Journal of Qualitative Methods. 3(1), pp. 42-55. doi: org/10.1177/160940690400300104

Harms, T., Clifford, R. M., \& Cryer, D. (2015). Early childhood environment rating scale ( $3^{\text {rd }}$ ed.). Teachers College Press.

Harvard University. (2017). Serve \& Return Interaction Shapes Brain Circuitry. http://developingchild.harvard.edu/resourcetag/serve-and-return/ 
Head Start ECLKC. (2013). Improving teacher-child interactions: Using the CLASS in Head Start preschool programs. https://eclkc.ohs.acf.hhs.gov/sites/default/files/pdf/using-theclass-in-hs-preschool-programs.pdf

Head Start ECLKC. (2020). A national overview of grantee CLASS scores in 2019. https://eclkc.ohs.acf.hhs.gov/data-ongoing-monitoring/article/national-overview-granteeclass-scores-2019

Head Start ECLKC. (2020) Teaching practices. https://eclkc.ohs.acf.hhs.gov/teachingpractices/article/promoting-adult-child-interactions-support-higher-order-thinkinglanguage-skills

Helburn, S. W. (Ed.). (1995). Cost, quality, and child outcomes study, technical report. University of Colorado at Denver.

Helm, J. H., \& Katz, L. (2001). Young investigators: The project approach in the early years. Teacher's College Press.

Hollway, W., \& Jefferson, T. (2000). Doing qualitative research differently: Free association, narrative, and the interview method. Sage Publications.

House of Representatives (n.d.). H.R.1-No Child Left Behind Act of 2001. https://www.congress.gov/bill/107th-congress/house-bill/1

Husserl, E. (1970). The crisis of European sciences and transcendental phenomenology. Northwestern University Press.

Illinois DCFS. (2016). Child Care Licensing Standards for Centers. https://www2.illinois.gov/dcfs/aboutus/notices/documents/rules_407.pdf 
Illinois Department of Human Services. (n.d.) FY2016 Illinois child care report.

http://www.dhs.state.il.us/OneNetLibrary/27897/documents/Child\%20Care/IllinoisAnnua 1ChildCareReportFY2016.pdf

Illinois Department of Human Services. (n.d.). Illinois Salary and Staffing Survey of Licensed Child Care Facilities Fiscal Year 2015. http://www.dhs.state.il.us/OneNetLibrary/27897/documents/HCD\%20Reports/Child\%20 Care/IllinoisSalaryandStaffingSurveyofLicensedChildCareFacilitiesFY2015.pdf

Illinois Professional Teaching Standards (IPTS) (2013). http://www.isbe.net/peac/pdf/il_prof_teaching_stds.pdf

Illinois State Board of Education. (2013). Illinois Early Learning and Development Standards (IELDS). http://isbe.net/earlychi/preschool/default.htm\#ields

Illinois State Board of Education. (2016). Illinois preschool for all: Early childhood block grant program for children ages 3 to 5 triennial report, fiscal years 2013, 2014, 2015. https://www.isbe.net/Documents/preschool13-15.pdf

Illinois State Board of Education Data Analysis Division. (2015). Illinois teacher salary study 2014-2015. http://www.isbe.net/research/pdfs/teacher_salary_14-15.pdf

Institute for Education Statistics (IES). (2009). Characteristics of public, private, and Bureau of Indian-education elementary and secondary school teachers in the United States: Results from the 2007-08 schools and staffing survey. https://nces.ed.gov/pubs2009/2009324/tables/sass0708_2009324_t12n_02.asp Institute of Medicine (IOM), \& National Research Council (NRC). (2015). Transforming the workforce for children birth through age 8: A unifying foundation. The National Academies Press. doi: 10.17226/19401 
Jalongo, M. R., Fennimore, B. S., Pattnaik, J., Laverick, J. B., \& Mutuku, M. (2004). Blended perspectives: A global vision for high-quality early childhood education. Early Childhood Education Journal, 32(3), 143-155. doi:

10.1023/B:ECEJ.0000048966.13626.be

Jordan, B., \& Smorti, S. (2010). Fearless science in the early years: Co-construction in a rural child care centre. The International Journal of Science in Society, 1(4), 85-104.

Karpov, Y. V. (2003). Development through the lifespan: A neo-Vygotskian approach. In Kozulin, A., Gindis, B., Ageyev, V. S., \& Miller, S. (Eds.), Vygotsky’s educational theory in cultural context (pp. 138-155). Cambridge University Press.

Karpov, Y. V. (2005). The neo-Vygotskian approach to child development. Cambridge University Press.

Kemmis, S., \& McTaggart, R. (Eds.). (1988). The action planner (3 ${ }^{\text {rd }}$ ed.). Deakin University Press.

Kohn, A. (1993). Punished by rewards: The trouble with gold stars, incentive plans, A's, praise, and other bribes. Houghton Mifflin Co.

Kostelnik, M. J., Soderman, A. K., \& Whiren, A. P. (2015). Developmentally appropriate curriculum (6 ${ }^{\text {th }}$ ed.). Pearson Merrill Prentice Hall.

Kozulin, A. (1986). Introduction. Thought and language. (pp. xi-lvi). MIT Press.

Kozulin, A. (2003). Psychological tools and mediated learning. In Kozulin, A., Gindis, B., Ageyev, V. S., \& Miller, S. (Eds.), Vygotsky's educational theory in cultural context (pp. 15-38). Cambridge University Press.

Kozulin, A., Gindis, B., Ageyev, V. S., \& Miller, S. M. (Eds.). (2003). Vygotsky's educational theory in cultural context. Cambridge University Press. 
Krichevets, A. N. (2014). Vygotsky and intersubjectivity. Psychology in Russia: State of the Art. $7(3), 13-23$.

LaJoie, S. (2005). Extending the scaffolding metaphor. Instructional Science, 33, 541-557. doi: $10.1007 / \mathrm{s} 11251-005-1279-2$

Lüdtke, U. M. (2012). Relational emotions in semiotic and linguistic development: Towards an intersubjective theory of language learning and language therapy. In A. Foolen, U. M. Lüdtke, T. P. Racine, \& J. Zlatev (Eds.), Moving ourselves, moving others: Motion and emotion in intersubjectivity, consciousness and language. John Benjamins, Amsterdam, The Netherlands.

Luke, A., \& Kale, J. (1997). Learning through difference: Cultural practices in early childhood language socialization. In E. Gregory (ed.). One child, many worlds: Early learning in multicultural communities (chapter 2). David Fulton Publishers.

Luria, A. R. (1982). Language and cognition. V. H. Winston \& Sons.

Mahn, H. (2003). Periods in child development: Vgotsky’s perspective. In Kozulin, A., Gindis, B., Ageyev, V. S., \& Miller, S. (Eds.), Vygotsky's educational theory in cultural context (pp. 119-137). Cambridge University Press.

Mayesky, M. (2011). Creative activities for young children $\left(10^{\text {th }}\right.$ ed.). Wadsworth.

McClelland, M. M., Cameron, C. E., Connor, C. M., Farris, C. L., Jewkes, A. M., \& Morrison, F. J. (2007). Links between behavioral regulation and preschoolers' literacy, vocabulary, and math skills. Developmental Psychology. 43(4), 947-959. doi: 10.1037/0012-

1649.43.4.947 
McMullen, M. B., \& Alat, K. (2002). Education matters in the nurturing of the beliefs of preschool teachers and caregivers. Early Childhood Research, 4(2). http://ecrp.uiuc.edu/v4n2/momullen.html

Merriam, S. B. (2009). Qualitative research: A guide to design and implementation. Jossey-Bass. Merriam-Webster Dictionary-Thesaurus. (2017). Dialogue. http://www.merriamwebster.com/dictionary/dialogue

Merriam-Webster Dictionary. (2017). Semiotics. http://www.merriamwebster.com/dictionary/semiotics

Merriam-Webster Dictionary. (2018). Frustration. https://www.merriamwebster.com/dictionary/frustration

Miles, M. B., Huberman, A. M., \& Saldaña, J. (2014). Qualitative data analysis: A methods sourcebook ( ${ }^{\text {rd }}$ ed.). Sage.

Moran, D. (2000). Introduction to phenomenology. Routledge.

Morrison, G. S. (2018). Early childhood education today. (14 ${ }^{\text {th }}$ ed.). Pearson.

Moss, P. (2006). Structures, understandings and discourses: Possibilities for re-envisioning the early childhood worker. Contemporary Issues in Early Childhood, 7(1), 30-41. doi: 10.2304/ciec.2006.7.1.30

Müller, N. (2003). Intelligibility and negotiated meaning in interaction. Clinical Linguistics and Phonetics, 17(4-5), 317-324.

National Association for the Education of Young Children. (n.d.). www.naeyc.org

National Association for the Education of Young Children. (n.d.). NAEYC accredited programs. http://families.naeyc.org/find-quality-child-care 
National Association for the Education of Young Children (n.d.). Developmentally Appropriate Practice. https://www.naeyc.org/resources/topics/dap

National Association for the Education of Young Children. (n.d.). Our mission and strategic position. www.naeyc.org/about/mission

National Association for the Education of Young Children. (n.d.). Side-by-side of the 2010 NAEYC professional preparation standards and the professional standards and competencies for early childhood educators. https://www.naeyc.org/sites/default/files/globallyshared/downloads/PDFs/resources/posit ion-statements/side_by_side_standards_comparison.pdf

National Research Council \& Institute of Medicine. (2000). From neurons to neighborhoods: The science of early childhood development. National Academy Press.

Newman, D., Griffin, P., \& Cole, M. (1989). The construction zone: Working for cognitive change in school. Cambridge University Press.

Norton, L., \& Arnold, L. (2021). Editorial. Educational Action Research, 29(2). 167-172, doi: 10.1080/09650792.2021.1890172

Organisation for Economic Cooperation and Development (OECD). (2016). Encouraging Quality in Early Childhood Education and Care (ECEC). http://www.oecd.org/education/school/49322232.pdf

Palinscsar, A. S. (1986). The role of dialogue in providing scaffolded instruction. Educational Psychologist, 21(1\& 2). 73-98.

Pence Turnbull, K. L., \& Justice, K. M. (2017). Language development from theory to practice ( $3^{\text {rd }}$ ed.). Pearson. 
Pianta, R., LaParo, K. M., \& Hamre, B. K. (2008). Classroom Assessment Scoring System: Manual Pre-K. Paul H. Brookes Publishing Company.

Portes, P. R., \& Vadeboncoeur, J. A. (2003). Mediation in cognitive socialization: The influence of socioeconomic status (pp. 371-392). Cambridge University Press.

Pronin, E. (2007). Perception and misperception of bias in human judgment. Trends in Cognitive Sciences, 11(1), 37-43. doi: 10.1016/j.tics.2006.11.001

Rochat, P., Passos-Ferreira, C., \& Salem, P. (2009, February). Three levels of intersubjectivity in early development. In A. Carassa, F. Morganti, \& G. Riva (Eds.). Enacting intersubjectivity: Paving the way for a dialogue between cognitive science, social cognition, and neuroscience (pp. 173-190). Oral session conducted at the International Workshop, organized by Istituto di Psicologia e Sociologia della Comunicazione, Lugano, Switzerland.

Rodriguez, C., \& Moro, C. (2008). Coming to agreement: Object use by infants and adults. In J. Zlatev, T. P. Racine, C. Sinha, and E. Itkonen (Eds.). The shared mind: Perspectives on intersubjectivity (pp. 89-114). John Benjamins.

Rogoff, B. (1990). Apprenticeship in thinking. Oxford University Press.

Rogoff, B. (2003). The cultural nature of human development. Oxford University Press.

Rogoff, B. (2008). Observing cultural-historical activity on three planes: Participatory appropriation, guided participation, and apprenticeship. Pedagogy and practice: Culture and identities, 58-74.

Rogoff, B., Mosier, C., Mistry, J., \& Göncü, A. (1993). Toddlers' guided participation with their caregivers in cultural activity. In E. A. Forman, N. Minick, \& C. A. Stone (Eds.), Contexts for learning (pp. 230-253). Oxford University Press. 
Searle, D. (1984). Scaffolding: Who's building whose building? Language Arts, 61(5), 480-483.

Shonkoff, J. P., \& Phillips, D. A. (Eds.) (2000). From neurons to neighborhoods: The science of early childhood development. National Academies Press.

Shore, R. (1997). Rethinking the brain: New insights into early development. Families to Work Institute.

Sinha, C., \& Rodriguez, C. (2008). Language and the signifying object: From convention to imagination. In J. Zlatev, T. P. Racine, C. Sinha, \& E. Itkonen (Eds.), The shared mind: Perspectives on intersubjectivity. (pp. 357-378). John Benjamins.

Stipek, D. (2006). No child left behind comes to preschool. The Elementary School Journal, 106(5), 455-466.

Stake, R. E. (1995). The art of case study research. Sage.

Stone, C. A. (1998). The metaphor of scaffolding: Its utility for the field of learning disabilities. Journal of Learning Disabilities, 31(4), 344-364.

Stringer, E. (1999). Action research: A handbook for practitioners ( $2^{\text {nd }}$ ed.). Sage.

Thompson, M. G. (2005). Phenomenology of intersubjectivity: A historical overview of the concept and its clinical implications. In J. Mills (Ed.), Relational and intersubjective perspectives in psychoanalysis: A critique (pp. 35-70). Rowman, Littlefield Publishers, Inc.

Trevarthen, C. (2008). Foreword. The shared mind: Perspectives on intersubjectivity. (pp. viixiii). John Benjamins.

Trevarthen, C., \& Aitken, K. J. (2001). Infant intersubjectivity: Research, theory, and clinical applications. Journal of Child Psychology and Psychiatry. 42(1), pp. 3-48. 
U.S. Department of Education. (2016). Fact Sheet: Troubling Pay Gap for Early Childhood Teachers. https://www.ed.gov/news/press-releases/fact-sheet-troubling-pay-gap-earlychildhood-teachers

U.S. Department of Education. (2016). Race to the Top--Early Learning Challenge. https://www2.ed.gov/programs/racetothetop-earlylearningchallenge/index.html

Valsiner, J. (1996). Co-constructionism and development: A socio-historic tradition. Anuario de Psicologia, 69, 63-82.

Valsiner, J., \& van der Veer, R. (1988). On the social nature of human cognition: An analysis of the shared intellectual roots of George Herbert Mead and Lev Vygotsky. Journal for the Theory of Social Behahior. 18(1), pp. 117-136.

Van der Veer, R., \& Valsiner, J. (1991). Understanding Vygotsky: A quest for synthesis. Basil Blackwell, Inc.

Vygotsky, L. S. (1978). Mind in society: The development of higher psychological processes. Harvard University Press.

Vygotsky, L. S. (1986). Thought and language. MIT Press.

Vygotsky, L. S. (1997a). Educational Psychology. CRC Press.

Vygotsky, L. S. (1997b). The collected works of L. S. Vygotsky Volume 4: The history of the development of higher mental functions. Plenum Press.

Vygotsky, L. S., and Luria, A. (1994). Tool and symbol in child development. In R. van der Veer \& J. Valsiner (Eds.). The Vygotsky reader (pp. 99-174), Blackwell Publishers.

Weikart, D.P. (1967/2016). Republication of "preschool programs: Preliminary findings. The Journal of Special Education, 50(1), 5-17. doi: 10.1177/0022466916637595

Wertsch, J. V. (1985). Vygotsky and the social formation of mind. Harvard University Press. 
Wertsch, J. V., \& Stone, C. A. (1985). The concept of internalization in Vygotsky's account of the genesis of higher mental functions. In J.V. Wertsch (ed.). Culture, communication, and cognition; Vygotskian Perspectives (pp. 162-179). Cambridge University Press.

WIDA (2021). https://wida.wisc.edu/about

Wojnar, D. M., \& Swanson, K. M. (2007). Phenomenology: An exploration. Journal of Holistic Nursing, 25(3), 172-180. doi: 10.1177/0898010106295172

Wood, D. (1999). Teaching the young child: Some relationships between social interaction, language, and thought. In P. V. Lloyd and C. Fernyhough (Eds.), Lev Vygotsky: Critical assessments, (vol. 3, pp. 259-275). Routledge.

Wood, D., Bruner, J. S., \& Ross, G. (1976). The role of tutoring in problem solving. Journal of Child Psychology and Psychiatry, 17, 89-100.

Zlatev, J. (2012). Prologue: Bodily motion, emotion and mind science. In A. Foolen, U. M. Lüdtke, T. P. Racine, \& J. Zlatev (eds.). Moving ourselves, moving others: Motion and emotion in intersubjectivity, consciousness and language (pp. 1-25). John Benjamins.

Zlatev, J., Racine, T. P., Sinha, C., \& Itkonen, E. (Eds.). (2008). The shared mind: Perspectives on intersubjectivity. John Benjamins.

Zumwalt, C. (2015, April). State panel presentation. [Forum]. Bloomington, IL. 


\section{APPENDIX A: INTERVIEW ONE SCRIPT AND QUESTIONS}

\section{SEMI-STRUCTURED INTERVIEW I}

Interview: How are you today? Thank you for meeting with me to discuss interactions with children.

1. Tell me about yourself and how you came to work here, in this class, with this age group.

a. Follow up if needed to get certificate, degree, years in ECE, years in preschool, ages of children in this classroom

2. What information do you want or need to know about your students to be an effective teacher?

a. Where do you get this information?

b. How is this information helpful to you; i.e. how do you use it?

3. Tell me about your teaching style (direct instruction and child-directed instruction)

a. What materials do you like to have and use in your classroom?

b. How do you engage children in learning? You can answer using examples.

4. What are your feelings and knowledge about 'guided participation', aka 'mediated activity?'

a. How do you include this method of teaching in your classroom? Examples?

5. How does your role as teacher influence a child's cognitive development?

6. Describe the many ways you communicate with your students, especially during instruction?

7. Why do you employ these methods of communication?

a. Does a child's culture factor in on your use of different communication methods? If so, please describe how.

8. When you engage a child in instruction, how do you determine or plan learning objectives?

a. How do you determine a child's readiness for that objective? Examples?

b. During instruction, how do you communicate with a child toward that objective? How long? Examples?

c. How do you determine whether a child met that objective? Examples?

9. How do you know whether a child understands you? Examples?

10. Do you have questions for me? 


\section{APPENDIX B: INTERVIEW TWO AND THREE SCRIPT AND QUESTIONS}

\section{SEMI-STRUCTURED INTERVIEW TWO AND THREE}

1. Based on your interactions with the child(ren), it seems you used (indicate) direct instruction/guided participation/ a combination of both during this learning experience. What led you in this instructional direction?

2. How do you feel the child was cognitively introduced to the activity?

a. Did you reference the child's life experiences (prior knowledge)?

3. What led you to begin the activity at that level of competence?

4. I see your use of the following communication methods (facial expression, tone, gestures, demonstration, materials, etc.). How did you perceive the child's reception of communication using these varied methods? (reference video)

a. Can we find evidence of serve and return? Joint attention? Other non-verbal give and take?

5. How did you incorporate the child's culture (life experiences) during instruction?

6. How did you determine whether the child understood you during the experience?

a. How did you use that understanding to extend the child's understanding of the concept presented?

7. How did you understand the child's perspective and level of knowledge during the experience?

a. How did you use that understanding to extend the child's understanding of the concept presented?

8. How do you feel CLASS trainings and practices help you and your students understand each other throughout instruction?

9. Do you have questions for me? 